\title{
THE ABSTRACT TIME-DEPENDENT CAUCHY PROBLEM
}

\author{
BY \\ MATTHEW HACKMAN
}

Preface. This paper treats existence of elementary solutions $B(t)$ for equations $B^{\prime}(t)=A(t) B(t)$, where $A(t)$ is an unbounded operator in a Banach space. Two points of view predominate. One is that generalization of the power-series definition of the exponential function is more fruitful than generalization of the product-integral definition $\left(e^{t}=\lim _{n \rightarrow \infty}(1+t / n)^{n}\right)$. The other point of view is that smoothing of unbounded operators exclusively by resolvents (particularly in the time-dependent case) leads to drastic losses in generality with little gain in clarity and simplicity.

Chapter one of the paper is purely introductory. It develops essential measuretheoretic preliminaries which are particularly relevant to the work of the rest of the paper and do not appear to be readily available elsewhere.

Chapter two begins the paper proper. It introduces the information about elementary solutions for the case of bounded operators from which the rest of the paper is developed. In addition to treating existence, uniqueness, and continuity, it includes a special growth estimate for elementary solutions that makes possible certain of the applications of the general theorems for unbounded operators proved later.

Chapter three is in a sense the core of the paper. Its main theorems are decidedly technical in nature, reflecting the unabashedly anatomical outlook taken toward the work of Hille, Kato, Phillips, and some others. These theorems comprise the first part of the chapter. The second part of the chapter is a justification of the first part. It rederives some of the work of Kato from the previous theorems of the chapter, and it exhibits some interesting nontrivial further applications of the theorems to cases not at all covered by the work of Kato inasmuch as projections in Hilbert space take the place of resolvents for smoothing.

Chapter four is inspired by work and suggestions of I. E. Segal. If the operators involved are affiliated with a ring of operators in a Hilbert space, then some ringtheoretic conditions on the operators ensuring adequate smoothing projections for construction of elementary solutions are available. One of the two cases basically considered may be applied to operators which are matrices in constant-coefficient

Presented to the Society, April 20, 1964 under the title Exponentiation of measurable operators and April 12, 1965 under the title The abstract time-dependent Cauchy problem for measurable operators affiliated with a discrete ring of operators in a separable Hilbert space; received by the editors February 14, 1964 and, in revised form, March 15, 1967. 
differential operators. The other case may be applied to exponentiation of operators affiliated with factors of type II.

Chapter five treats briefly a possible direction in which one may proceed if the operators involved are bad boundary-values of well-behaved analytic functions. The basic case it seeks to generalize is mentioned in the last paragraph of the chapter.

1. Banach space lemmas. Let $X$ be a separable Banach space.

(a) Definition. If $\Omega$ is a measure space, a function $x: \Omega \rightarrow X$ is called measurable if $f(x(\cdot))$ is measurable for all $f \in X^{*}$. A function $A: \Omega \rightarrow B(X)$ is called measurable if $A(\cdot) x$ is measurable for all $x \in X$.

The measurable functions from $\Omega$ to $X$ and from $\Omega$ to $B(X)$, respectively, form vector spaces.

Let $\left\{x_{n}\right\}$ be a sequence dense in $X$. Let $\left\{f_{n}\right\} \subset X^{*}$ be such that $\left\|f_{n}\right\|=1$, and $f_{n}\left(x_{n}\right)=\left\|x_{n}\right\|$. Then if $x: \Omega \rightarrow X$ is measurable, $\|x(\omega)\|=\operatorname{lub}_{n=1, \ldots, \infty}\left|f_{n}(x(\omega))\right|$, so that $\|x(\cdot)\|$ is measurable. If $A: \Omega \rightarrow B(X)$ is measurable, then $\|A(\omega)\|=$ $\operatorname{lub}_{n=1, \ldots, \infty}\left(\left\|A(\omega) x_{n}\right\|\right) /\left(\left\|x_{n}\right\|\right)$ is measurable.

We wish to show that the measurable functions from $\Omega$ to $B(X)$ form an algebra. When $X$ is a Hilbert space this is easy. Let $A(\cdot)$ be measurable. Then $\|A(\cdot) x\|^{2}$ $=(A(\cdot) x, A(\cdot) x)$ is measurable for all $x \in X$. Then $2(A(\cdot) x, A(\cdot) y)=\|A(\cdot)(x+y)\|^{2}$ $+i\|A(\cdot)(x+i y)\|^{2}-(1+i)\|A(\cdot) x\|^{2}-(1+i)\|A(\cdot) y\|^{2}$ is measurable for all $x$ and $y$ in $X$, so that $A(\cdot)^{*} A(\cdot)$ is measurable. Since $A(\cdot)^{*}$ is trivially measurable whenever $A(\cdot)$ is, $A(\cdot)=S(\cdot)+i T(\cdot)$ with $S(\cdot)$ and $T(\cdot)$ self-adjoint and measurable. Thus we need only show that $A(\cdot) B(\cdot)$ is measurable whenever $A(\cdot)$ and $B(\cdot)$ are selfadjoint and measurable. But if $A(\cdot)$ and $B(\cdot)$ are measurable, then $(A(\cdot)+B(\cdot))^{*}(A(\cdot)+B(\cdot))=A(\cdot)^{2}+B(\cdot)^{2}+A(\cdot) B(\cdot)+B(\cdot) A(\cdot)$ is measurable, so that $A(\cdot) B(\cdot)+B(\cdot) A(\cdot)$ is measurable. Similarly, $(A(\cdot)+i B(\cdot))^{*}(A(\cdot)+i B(\cdot))$ $=A(\cdot)^{2}+B(\cdot)^{2}+i A(\cdot) B(\cdot)-i B(\cdot) A(\cdot)$ is measurable, so that $A(\cdot) B(\cdot)-B(\cdot) A(\cdot)$ is measurable. Thus $A(\cdot) B(\cdot)$ is measurable.

More generally, when $X$ is not necessarily a Hilbert space, $A(\cdot) B(\cdot)$ is still measurable when both $A(\cdot)$ and $B(\cdot)$ are. Since $A(\cdot) B(\cdot)$ measurable means that $A(\cdot) B(\cdot) x$ is measurable for all $x \in X$, it suffices to show that $A(\cdot) x(\cdot)$ is measurable whenever $A(\cdot)$ and $x(\cdot)$ both are. Certainly if $x_{n}(\omega) \rightarrow x(\omega)$ in the norm for each $\omega$ and if $A(\cdot) x_{n}(\cdot)$ is measurable for each $n$, then $A(\cdot) x(\cdot)$ is measurable. If $x_{n}(\cdot)$ were measurable and countably-valued, then $A(\cdot) x_{n}(\cdot)$ would be measurable. But by a standard result $x(\cdot)$ can in fact be approximated by a sequence $\left\{x_{n}(\cdot)\right\}$ of countablyvalued measurable $x_{n}(\cdot)$ for which $\left\|x(\omega)-x_{n}(\omega)\right\| \rightarrow 0$ uniformly in $\omega$.

(b) We make the following definitions for convenience.

DEFINITION. $\mathscr{M}(X, \Omega)$ denotes the space of measurable functions from $\Omega$ into $X$. $\mathscr{M}(B(X), \Omega)$ denotes the algebra of measurable functions from $\Omega$ into $B(X)$.

DEFINITION.

$$
L^{p}(X, \Omega)=\left\{x(\cdot) \in \mathscr{M}(X, \Omega):\|x(\cdot)\| \in L^{p}(\Omega)\right\}
$$


and

$$
L^{p}(B(X), \Omega)=\left\{A(\cdot) \in \mathscr{M}(B(X), \Omega):\|A(\cdot)\| \in L^{p}(\Omega)\right\}
$$

For $x(\cdot) \in L^{p}(X, \Omega)$, we set $\|x(\cdot)\|_{p}=\left(\int_{\Omega}\|x(\cdot)\|^{p}\right)^{(1 / p)}$, and for $A(\cdot) \in L^{p}(B(X), \Omega)$, we set $\|A(\cdot)\|_{p}=\left(\int_{\Omega}\|A(\cdot)\|^{p}\right)^{(1 / p)}$.

The spaces $L^{p}(X, \Omega)$ and $L^{p}(B(X), \Omega)$ are Banach spaces for $p \geqq 1 . L^{1}(X, \Omega)$ is precisely the space of Bochner-integrable functions from $\Omega$ into $X$. Unfortunately, only those $A(\cdot) \in L^{1}(B(X), \Omega)$ which are "almost separably-valued" are Bochner integrable with $B(X)$ considered as a Banach space under the operator norm. (For $A(\cdot) \in \mathscr{M}(B(X), \Omega), A(\cdot)$ is almost separably-valued if there exists a null-set $N$ such that $\{A(\omega): \omega \in \Omega-N\}$ is contained in a separable subspace of the Banach space $B(X)$.) For example, if $\Omega$ happens to be a topological space with a countable basis of open sets, then $A(\cdot)$ is almost separably-valued if it is almost everywhere continuous as a function from $\Omega$ to the Banach space $B(X)$.

(c) We specialize to the case that $\Omega=[a, b]$, a bounded interval with Lebesgue measure.

(1) Definition. A function $x(\cdot):[a, b] \rightarrow X$ is of bounded variation if $\exists K>0$ such that $\sum_{i=1}^{n}\left\|x\left(t_{i}\right)-x\left(t_{i-1}\right)\right\|<K$ for all choices of $a=t_{0}<t_{1}<\cdots<t_{n}=b$. A function $A(\cdot):[a, b] \rightarrow B(X)$ is of bounded variation if $\exists K>0$ such that $\sum_{i=1}^{n}\left\|A\left(t_{i}\right)-A\left(t_{i-1}\right)\right\|<K$ for all choices of $a=t_{0}<t_{1}<\cdots<t_{n}=b$.

Since complex-valued functions of bounded variation are measurable, application of continuous linear functionals shows that $x(\cdot)$ is measurable if it is of bounded variation. Directly, $A(\cdot)$ is measurable if it is of bounded variation. Even more, since it is continuous on $[a, b]$ except at a countable number of points, it is almost separably-valued.

For $A(\cdot)$ to be of bounded variation, it suffices to know that $A(\cdot) x$ is of bounded variation $\forall x \in X$. To see this define a continuous pseudo-norm on $X$ by $\rho_{S}=$ $\sum_{i=1}^{n}\left\|A\left(t_{i}\right) x-A\left(t_{i-1}\right) x\right\|$, where $S$ represents the subdivision $a=t_{0}<\cdots<t_{n}=b$. Then $\rho_{S}$ is uniformly bounded on each $x$, so that there exists $c$ with $\rho_{S}(x) \leqq c\|x\|$ for all $x \in X$ by Bourbaki's version of the Banach-Steinhaus theorem. Thus for all subdivisions of $[a, b], \sum_{i=1}^{n}\left\|A\left(t_{i}\right)-A\left(t_{i-1}\right)\right\| \leqq c$.

(2) Definition. A function $x(\cdot):[a, b] \rightarrow X$ is absolutely continuous if $\forall \varepsilon>0$, $\exists \delta>0$ such that $\sum_{i=1}^{n}\left\|x\left(b_{i}\right)-x\left(a_{i}\right)\right\|<\varepsilon$ whenever $\left\{\left(a_{i}, b_{i}\right)\right\}_{i=1}^{n}$ is a collection of disjoint open subintervals of $[a, b]$ with $\sum_{i=1}^{n}\left(b_{i}-a_{i}\right)<\delta$. A function $A(\cdot):[a, b] \rightarrow B(X)$ is absolutely continuous if a similar condition holds with $\sum_{i=1}^{n}\left\|A\left(b_{i}\right)-A\left(a_{i}\right)\right\|$ replacing $\sum_{i=1}^{n}\left\|x\left(b_{i}\right)-x\left(a_{i}\right)\right\|$.

Since absolute continuity implies continuity, $x(\cdot)$ and $A(\cdot)$ are uniformly bounded on $[a, b]$ if they are absolutely continuous. Directly, $A(\cdot) x(\cdot)$ is absolutely continuous if $A(\cdot)$ and $x(\cdot)$ are each absolutely continuous, and $A(\cdot) B(\cdot)$ is absolutely continuous if $A(\cdot)$ and $B(\cdot)$ each are. Parenthetically, we note that absolutely continuous functions are automatically of bounded variation.

If $A(\cdot) \in L^{1}(B(X),[a, b])$ and is, moreover, almost separably-valued, then 
$B(t)=\int_{a}^{t} A(s) d s$ is absolutely continuous on $[a, b]$, and $B(t)$ is uniformly differentiable a.e. on $[a, b]$ with derivative $B^{\prime}(t)=A(t)$. If $B_{1}(\cdot)$ and $B_{2}(\cdot)$ are absolutely continuous and are uniformly differentiable a.e. on $[a, b]$, then so is $B_{1}(\cdot) B_{2}(\cdot)$, and $\left(B_{1}(t) B_{2}(t)\right)^{\prime}=B_{1}^{\prime}(t) B_{2}(t)+B_{1}(t) B_{2}^{\prime}(t)$ a.e. on $[a, b]$.

If, however, $A(\cdot) \in L^{1}(B(X),[a, b])$ is not necessarily almost separably-valued, we know only that there exists $B(\cdot)$ with $B(t) x=\int_{a}^{t} A(s) x d s \quad \forall x \in X$. This means that $\left\|\left(B\left(t_{2}\right)-B\left(t_{1}\right)\right) x\right\| \leqq\|x\| \int_{t_{1}}^{t_{2}}\|A(s)\| d s \quad \forall x \in X$, so that $\left\|B\left(t_{2}\right)-B\left(t_{1}\right)\right\| \leqq$ $\int_{t_{1}}^{t_{2}}\|A(s)\| d s$. Thus $\|B(t)\| \leqq\|A(\cdot)\|_{1} \quad \forall t \in[a, b], B(\cdot)$ is absolutely continuous on $[a, b]$, and since $\int_{a}^{t}\|A(s)\| d s$ is differentiable for almost all $t \in[a, b]$, $(1 / \delta)\|B(t+\delta)-B(t)\|$ and $(1 / \delta)\|B(t)-B(t-\delta)\|$ are bounded uniformly in $\delta$ for almost all $t \in[a, b]$.

For $x(\cdot) \in L^{1}(X,[a, b]), \int_{a}^{t} x(s) d s$ is strongly differentiable for almost all $t \in[a, b]$ with $\left(\int_{a}^{t} x(s) d s\right)^{\prime}=x(t)$. Let $A_{1}(\cdot)$ and $A_{2}(\cdot) \in L^{1}(B(X),[a, b])$. Setting $B_{i}(t) x$ $=\int_{a}^{t} A_{i}(s) x d s(i=1,2)$, we may assert that, for each $x \in X, B_{i}(t) x$ is strongly differentiable for almost all $t \in[a, b]$ with $\left(B_{i}(t) x\right)^{\prime}=A_{i}(t) x$. We should like to assert that $B_{1}(t) B_{2}(t) x$ is strongly differentiable for almost all $t \in[a, b]$ with $\left(B_{1}(t) B_{2}(t) x\right)^{\prime}=A_{1}(t) B_{2}(t) x+B_{1}(t) A_{2}(t) x$. The following lemma suffices to establish this.

Lemma. Let $x(\cdot)$ be strongly differentiable a.e. on $[a, b]$ with derivative $x^{\prime}(\cdot)$. Let $A(\cdot) \in L^{1}(B(X),[a, b])$, and let $B(t) x=\int_{a}^{t} A(s) x d s \quad \forall x \in X$. Then $B(t) x(t)$ is strongly differentiable a.e. on $[a, b]$ with derivative $A(t) x(t)+B(t) x^{\prime}(t)$.

Proof. Let $N_{0}$ be a null set such that $x(\cdot)$ is differentiable $\forall t \in[a, b]-N_{0}$ with derivative at $t$ equal to $x^{\prime}(t)$. Let $N_{00}$ be a null set such that $\int_{a}^{t}\|A(s)\| d s$ is differentiable $\forall t \in[a, b]-N_{00}$ with derivative at $t$ equal to $\|A(t)\|$. If $\left\{x_{n}\right\}$ is a sequence dense in $X$, then for each $x_{n}$ the set of $t$ for which $B(t) x_{n}$ is differentiable with $\left(B(t) x_{n}\right)^{\prime}$ equal to $A(t) x_{n}$ is contained in $[a, b]$ minus a null set $N_{n}$.

Set $N=N_{00} \cup \bigcup_{n=0}^{\infty} N_{n}$, and let $t \in[a, b]-N$. Then

$$
\begin{aligned}
&\left\|\left(A(t) x(t)+B(t) x^{\prime}(t)\right)-(1 / \delta)(B(t+\delta) x(t+\delta)-B(t) x(t))\right\| \\
& \leqq\|(A(t)-(1 / \delta)(B(t+\delta)-B(t))) x(t)\| \\
&+\|B(t+\delta)\| \cdot\left\|x^{\prime}(t)-(1 / \delta)(x(t+\delta)-x(t))\right\| \\
&+\|B(t)-B(t+\delta)\| \cdot\left\|x^{\prime}(t)\right\| .
\end{aligned}
$$

Certainly the last two terms go to zero with $\delta$. The first term is majorized by

$$
\begin{aligned}
& \left\|(A(t)-(1 / \delta)(B(t+\delta)-B(t))) x_{n}\right\| \\
& \quad+\|A(t)\| \cdot\left\|x_{n}-x(t)\right\|+(1 / \delta)\|B(t+\delta)-B(t)\| \cdot\left\|x_{n}-x(t)\right\| .
\end{aligned}
$$

But we may choose $x_{n}$ to make the last two terms of this uniformly small in $\delta$, and then let $\delta$ go to zero to make the first term vanish.

Suppose now $x(\cdot)$ is absolutely continuous on $[a, b]$ and $(f(x(\cdot)))^{\prime}=0$ a.e. $\forall f \in X^{*}$. Then $x(\cdot)$ is constant. 
When $x(\cdot)$ is of bounded variation on $[a, b]$ and has a weak derivative $x^{\prime}(\cdot)$ a.e. on $[a, b], x^{\prime}(\cdot) \in L^{1}(X,[a, b])$ by a standard theorem of vector integration. In particular this is true if $x(\cdot)$ is absolutely continuous on $[a, b]$ and has a weak derivative $x^{\prime}(\cdot)$ a.e. But by the above paragraph $x(t)-\int_{a}^{t} x^{\prime}(s) d s$ is then constant, so that $x(t)=x(a)+\int_{a}^{t} x^{\prime}(s) d s$, and $x(\cdot)$ is even strongly differentiable a.e. on $[a, b]$.

2. The Cauchy problem for bounded operators in a separable Banach space.

(a) Lemma 1. Let $A(\cdot) \in L^{1}(B(X),[a, b])$. Then there exist $B(\cdot, a)$ and $\bar{B}(\cdot, a) \in L^{\infty}(B(X),[a, b])$ such that

(1) $\|B(t, a)-I\| \leqq \exp \left(\int_{a}^{t}\|A(s)\| d s\right)-1$ and $\|\bar{B}(t, a)-I\| \leqq \exp \left(\int_{a}^{t}\|A(s)\| d s\right)-1$, and (2) $(B(t, a)-I) x=\int_{a}^{t} A(s) B(s, a) x d s$ and $(\bar{B}(t, a)-I) x=-\int_{a}^{t} \bar{B}(s, a) A(s) x d s$, for all $x \in X$.

If $A(\cdot)$ is almost separably-valued, then $B(\cdot, a)$ and $\bar{B}(\cdot, a)$ are also, and $(B(t, a)-I)=\int_{a}^{t} A(s) B(s, a) d s$, and $(\bar{B}(t, a)-I)=-\int_{a}^{t} \bar{B}(s, a) A(s) d s$.

Proof. Set $\alpha(t)=\|A(t)\|$. For any $n, A\left(t_{n}\right) A\left(t_{n-1}\right) \cdots A\left(t_{1}\right)$ is measurable on $[a, b]^{n}$, and, since $\left\|A\left(t_{n}\right) A\left(t_{n-1}\right) \cdots A\left(t_{1}\right)\right\| \leqq \alpha\left(t_{n}\right) \cdots \alpha\left(t_{1}\right)$,

$$
A\left(t_{n}\right) A\left(t_{n-1}\right) \cdots A\left(t_{1}\right) \in L^{1}\left(B(X),[a, b]^{n}\right) .
$$

For $t \in[a, b]$ let $B_{n}(t) \in B(X)$ be such that $\forall x \in X$,

$$
B_{n}(t) x=\int_{t \geqq t_{n} \geqq \cdots \geqq t_{1}=a} A\left(t_{n}\right) \cdots A\left(t_{1}\right) x d t_{1} \cdots d t_{n} .
$$

Then

$$
\begin{aligned}
\left\|B_{n}(t) x\right\| & \leqq\|x\| \int_{t \geq t_{n} \geq \cdots \geq t_{1}=a} \alpha\left(t_{n}\right) \cdots \alpha\left(t_{1}\right) d t_{1} \cdots d t_{n} \\
& =\|x\| \int_{a}^{t} \alpha\left(t_{n}\right) d t_{n} \int_{a}^{t_{n}} \alpha\left(t_{n-1}\right) d t_{n-1} \int_{a}^{t_{2}} \alpha\left(t_{1}\right) d t_{1} .
\end{aligned}
$$

Set $\beta(t)=\int_{a}^{t} \alpha(s) d s$, so that $\beta^{\prime}(t)=\alpha(t)$ a.e. on $[a, b]$. Then

$$
\begin{aligned}
\left\|B_{n}(t)\right\| & \leqq \int_{a}^{t} \beta^{\prime}\left(t_{n}\right) d t_{n} \cdots \int_{a}^{t_{2}} \beta^{\prime}\left(t_{1}\right) d t_{1}=\int_{a}^{t} \beta^{\prime}\left(t_{n}\right) d t_{n} \cdots \int_{a}^{t_{3}} \beta\left(t_{2}\right) \beta^{\prime}\left(t_{2}\right) d t_{2} \\
& =\int_{a}^{t} \beta^{\prime}\left(t_{n}\right) d t_{n} \cdots \int_{a}^{t_{3}}\left(\beta\left(t_{3}\right)^{2} / 2 !\right) \beta^{\prime}\left(t_{3}\right) d t_{3}=\int_{a}^{t}\left(\beta\left(t_{n}\right)^{n-1} /(n-1) !\right) \beta^{\prime}\left(t_{n}\right) d t_{n} \\
& =\left(\beta(t)^{n} / n !\right) .
\end{aligned}
$$

If $f\left(t_{1}, \ldots, t_{n}\right)$ is any nonnegative function in $L^{1}\left([a, b]^{n}\right)$, then

$$
\int_{t \geq t_{n} \geq \cdots \geq t_{1}=a} f\left(t_{1}, \ldots, t_{n}\right) d t_{1} \cdots d t_{n}
$$

is a monotone increasing function of $t$ and, hence, is measurable. Thus

$$
\int_{t \geq t_{n} \geq \ldots \geq t_{1}=a} f\left(t_{1}, \ldots, t_{n}\right) d t_{1} \cdots d t_{n}
$$


is measurable for any $f\left(t_{1}, \ldots, t_{n}\right) \in L^{1}\left([a, b]^{n}\right)$. Thus $\forall f \in X^{*}$

$$
f\left(B_{n}(t) x\right)=\int_{t \geq t_{n} \geq \cdots \geqq t_{1} \geq a} f\left(A\left(t_{n}\right) \cdots A\left(t_{1}\right) x\right) d t_{1} \cdots d t_{n}
$$

is measurable, so that $B_{n}(\cdot) \in L^{\infty}(B(X),[a, b])$. Since $\left\|\sum_{k=m}^{n} B_{k}(\cdot)\right\|_{\infty}$ $\leqq \sum_{k=m}^{n}(\beta(b) / k !), \sum_{k=1}^{n} B_{k}(\cdot)$ is Cauchy in $L^{\infty}(B(X),[a, b])$.

Set $B(\cdot, a)-I=\sum_{k=1}^{\infty} B_{k}(\cdot)$. Then

$$
\|B(t, a)-I\| \leqq \sum_{k=1}^{\infty}\left\|B_{k}(t)\right\| \leqq \sum_{k=1}^{\infty}\left(\beta(t)^{k} / k !\right)=\exp (\beta(t))-1 .
$$

This proves the first assertion for $B(t, a)$. To prove the second assertion we note that

$$
\begin{aligned}
\left\|\sum_{k=1}^{n} A(t) B_{k}(t)\right\| & \leqq\|A(t)\| \sum_{k=1}^{n}\left(\beta(t)^{k} / k !\right) \\
& \leqq\|A(t)\| \sum_{k=1}^{n}\left(\beta(b)^{k} / k !\right) \leqq\|A(t)\|(\exp \beta(b)-1),
\end{aligned}
$$

so that for all $n$ the norm of $\sum_{k=1}^{n} A(t) B_{k}(t)$ is bounded by the function

$$
\|A(t)\|(\exp \beta(b)-1)
$$

which is in $L^{1}([a, b])$. Since $\sum_{k=1}^{n} B_{k}(t)$ is uniformly convergent for each $t \in[a, b]$, for each $x \in X$ we have

$$
\int_{a}^{t} A(s) B(s, a) x d s=\int_{a}^{t} A(s) x d s+\sum_{k=1}^{\infty} \int_{a}^{t} A(s) B_{k}(s) x d s .
$$

But

$$
\begin{aligned}
\int_{a}^{t} A(s) B_{k}(s) x d s & =\int_{a}^{t} A(s) d s \int_{s \geq_{k} \geq \cdots \geq_{s_{1}} \geq a} A\left(s_{k}\right) \cdots A\left(s_{1}\right) x d s_{1} \cdots d s_{k} \\
& =\int_{t \geq_{s} \geq_{s_{k}} \geq \cdots \geq_{s_{1}} \geq a} A(s) A\left(s_{k}\right) \cdots A\left(s_{1}\right) x d s_{1} \cdots d s_{k} d s
\end{aligned}
$$

by the Fubini theorem for Bochner integrals, $=B_{k+1}(t) x$. Thus

$$
\int_{a}^{t} A(s) B(s, a) x d s=\sum_{k=1}^{\infty} B_{k}(t) x=(B(t, a)-I) x
$$

All of the assertions for $\bar{B}(\cdot, a)$ are established in exactly the same manner except that $\bar{B}_{k}(t)$ is.defined by

$$
\bar{B}_{k}(t) x=(-1)^{n} \int_{t \geq t_{n} \geq \cdots \geq t_{1} \geq a} A\left(t_{1}\right) \cdots A\left(t_{n}\right) x d t_{1} \cdots d t_{n}, \quad \forall x \in X .
$$

When $A(\cdot)$ is almost separably-valued all of the relevant integrals may be taken directly as uniform Bochner integrals. The stronger assertions in this case follow immediately. 
Corollary. $B(\cdot, a)$ and $\bar{B}(\cdot, a)$ as defined above are absolutely continuous on $[a, b]$ and are strongly differentiable a.e. on $[a, b]$ on each $x \in X$ with derivatives $A(\cdot) B(\cdot, a)$ and $-\bar{B}(\cdot, a) A(\cdot)$, respectively. When $A(\cdot)$ is almost separably-valued, $B(\cdot, a)$ and $\bar{B}(\cdot, a)$ are, in addition, uniformly differentiable a.e. on $[a, b]$.

Proof. The corollary is immediate from the integral formulas and bounds for $(B(\cdot, a)-I)$ and $(\bar{B}(\cdot, a)-I)$.

LEMMA 2. $\bar{B}(t, a)$ is invertible $\forall t \in[a, b]$.

Proof. Let $a=a_{0}<a_{1}<\cdots<a_{n}=b$ be any subdivision of $[a, b]$ with

$$
\int_{a_{i}}^{a_{i+1}}\|A(s)\| d s<\frac{1}{2} \quad \forall i
$$

Then $\forall t \in\left[a_{i}, a_{i+1}\right]$,

$$
\begin{aligned}
\left\|B\left(t, a_{i}\right)-I\right\| & \leqq \exp \left(\int_{a_{i}}^{t}\|A(s)\| d s\right)-1 \\
& \leqq \exp \left(\int_{a_{i}}^{a_{i+1}}\|A(s)\| d s\right)-1<e^{1 / 2}-1<2-1=1 .
\end{aligned}
$$

Thus $B\left(t, a_{i}\right)$ is invertible $\forall t \in\left[a_{i}, a_{i+1}\right]$. Set $C(t)=B\left(t, a_{i}\right) B\left(a_{i}, a_{i-1}\right) \cdots B\left(a_{1}, a_{0}\right)$ for $t \in\left[a_{i}, a_{i+1}\right]$. Then $C(\cdot)$ is absolutely continuous on $[a, b]$, is strongly differentiable a.e. on $[a, b]$ with derivative $A(\cdot) C(\cdot)$, and is invertible everywhere on $[a, b]$.

But $\bar{B}(\cdot, a) C(\cdot) x$ is strongly differentiable a.e. on $[a, b]$ for each $x \in X$ with derivative $-\bar{B}(\cdot, a) A(\cdot) C(\cdot) x+\bar{B}(\cdot, a) A(\cdot) C(\cdot) x=0$ and is, in addition, absolutely continuous on $[a, b]$. Thus $\bar{B}(t, a) C(t) x=x \quad \forall t \in[a, b]$, so that $\bar{B}(t, a) C(t)$ $=I \quad \forall t \in[a, b]$, so that $\bar{B}(t, a)=C(t)^{-1} \quad \forall t \in a, b$.

COROLlaRY 1. If $x(\cdot)$ is absolutely continuous on $[a, b]$ and strongly differentiable a.e. with $x^{\prime}(\cdot)=A(\cdot) x(\cdot)$, then $x(t)=\bar{B}(t, a)^{-1} x(a) \quad \forall t \in[a, b]$.

Proof. $\bar{B}(\cdot, a) x(\cdot)$ is absolutely continuous on $[a, b]$ and is differentiable a.e. with derivative $-\bar{B}(\cdot, a) A(\cdot) x(\cdot)+\bar{B}(\cdot, a) A(\cdot) x(\cdot)=0$, so that $\bar{B}(t, a) x(t)=$ $\bar{B}(a, a) x(a)=x(a) \quad \forall t \in[a, b]$, so that $x(t)=\bar{B}(t, a)^{-1} x(a) \quad \forall t \in[a, b]$.

COROLlary 2. If $C(\cdot)$ is absolutely continuous on $[a, b]$ and if $C(\cdot) x$ is strongly differentiable a.e. $\forall x \in X$ with derivative $A(\cdot) C(\cdot) x$, then $C(\cdot)=\bar{B}(\cdot, a)^{-1} C(a)$.

Proof. Immediate from Corollary 1.

COROLlary 3. $B(\cdot, a)$ is the unique absolutely continuous function from $[a, b]$ to $B(X)$ such that $B(a, a)=I$ and such that $B(\cdot, a) x$ is strongly differentiable a.e. $\forall x \in X$ with derivative $A(\cdot) B(\cdot, a) x . \quad \bar{B}(\cdot, a)^{-1}=B(\cdot, a)$, so that $B(\cdot, a)$ is invertible everywhere on $[a, b]$.

Proof. Immediate from Corollary 2. 
Corollary 4. $B(t, a)=B(t, s) B(s, a) \quad \forall s \in[a, t]$. Thus

$$
B(s, a)=B(t, s)^{-1} B(t, a) \quad \forall t \in[s, b] .
$$

Proof. For $s \in[a, b]$, let $C(\cdot)$ be defined by $C(t)=B(t, a)$ for $a \leqq t \leqq s, C(t)$ $=B(t, s) B(s, a)$ for $s \leqq t \leqq b$. Then $C(\cdot)$ is absolutely continuous on $[a, b]$, and $C(\cdot) x$ is strongly differentiable a.e. with derivative $A(\cdot) C(\cdot) x$. Thus $C(\cdot)=B(\cdot, a)$, or $B(t, s) B(s, a)=B(t, a) \quad \forall t \in[s, b]$, i.e., $\forall s \in[a, t]$.

Corollary 5. $B(b, \cdot) x$ is differentiable a.e. on $[a, b]$ for all $x \in X$ with derivative $-B(b, \cdot) A(\cdot) x$. If $A(\cdot)$ is almost separably-valued, $B(b, \cdot)$ is uniformly differentiable a.e. on $[a, b]$.

Proof. By Corollary $4, B(b, a) B(\cdot, a)^{-1}=B(b, \cdot)$. Corollary 5 follows at once.

COROLlaRY 6. $B(\cdot, a)^{-1}$ is the unique absolutely continuous function from $[a, b]$ to $B(X)$ such that $B(a, a)^{-1}=I$ and such that $B(\cdot, a)^{-1} x$ is strongly differentiable a.e. $\forall x \in X$ with derivative $-B(\cdot, a) A(\cdot) x$.

Proof. Let $C(\cdot)$ be absolutely continuous on $[a, b]$, and let $C(\cdot) x$ be differentiable a.e. $\forall x \in X$ with derivative $-C(\cdot) A(\cdot) x$. Let $C(a)=I$. Then $(C(t)-I) x$ $=-\int_{a}^{t} C(s) A(s) x d s \quad \forall x \in X$. Then $C(\cdot) B(\cdot, a) x$ is strongly differentiable a.e. with derivative equal to zero, so that $C(t) B(t, a)=I$, so that $C(t)=B(t, a)^{-1}$.

(b) We have obtained a bound $\|B(t, a)\| \leqq\|B(t, a)-I\|+1 \leqq \exp \left(\int_{a}^{t}\|A(s)\| d s\right)$ which is by no means the best possible one. If, for example, $A(\cdot)=f(\cdot) I$, where $f(\cdot) \in L^{1}([a, b]), B(t, a)=\exp \left(\int_{a}^{t} f(s) d s\right) I$, so that $\|B(t, a)\|=\exp \left(\int_{a}^{t} \operatorname{Re} f(s) d s\right)$, the bound we have obtained is exact only if $\operatorname{Re} f(s)=|f(s)|$ a.e., i.e., only if $f(s)$ $\geqq 0$ a.e.

A bound for $\|B(t, a)\|$ which is exact in this case is, however, obtainable.

DEEINITION. For $A \in B(X), \rho(A)=\inf _{\alpha \geq 0}(\|A+\alpha\|-\alpha)$.

Since $\|A+\alpha\| \leqq\|A\|+\alpha$ and $\alpha \leqq\|A\|+\|A+\alpha\|,-\|A\| \leqq \rho(A) \leqq\|A\|$. If $\alpha_{1} \geqq \alpha_{2}$, then $\left\|A+\alpha_{1}\right\|-\alpha_{1}=\left\|A+\alpha_{2}+\left(\alpha_{1}-\alpha_{2}\right)\right\|-\alpha_{1} \leqq\left\|A+\alpha_{2}\right\|-\alpha_{2}$, so that $\|A+\alpha\|-\alpha \downarrow$, so that $\rho(A)=\lim _{n \rightarrow \infty}(\|A+n\|-n)$.

Clearly $\rho(0)=0$. If $\lambda>0,\|\lambda A+\lambda \alpha\|-\lambda \alpha=\lambda(\|A+\alpha\|-\alpha)$, so that $\rho(\lambda A)=\lambda \rho(A)$, and $\rho(\lambda A)=\lambda \rho(A)$ for $\lambda \geqq 0$. Also,

$$
\left\|A+B+\alpha_{1}+\alpha_{2}\right\|-\left(\alpha_{1}+\alpha_{2}\right) \leqq\left(\left\|A+\alpha_{1}\right\|-\alpha_{1}\right)+\left(\left\|B+\alpha_{2}\right\|-\alpha_{2}\right),
$$

so that $\rho(A+B) \leqq \rho(A)+\rho(B)$.

Further, if $a$ is any real number, then for $\alpha$ with $\alpha+a>0$,

$$
\|(A+a)+\alpha\|-\alpha=\|A+(a+\alpha)\|-(a+\alpha)+a,
$$

so that $\rho(A+a I)=\rho(A)+a$. If $a$ and $b$ are real numbers, then $\rho((a+b i) I)$ $=a+|b| \rho( \pm i I)$. But

$$
\| \pm i I+n\|-n=\left(1+n^{2}\right)^{1 / 2}-n \leqq\left(1+n^{2}+\left(1 / 4 n^{2}\right)\right)^{1 / 2}-n=(n+(1 / 2 n))-n=1 / 2 n \rightarrow 0
$$

as $n \rightarrow \infty$, so that $\rho((a+b i) I)=a$. 
Finally, if $\lambda>\rho(A)$, then $\exists n>0$ with $n+\lambda>0$. Then $\|A+n\|-n<\lambda$ implies $\|A+n\|<(n+\lambda)$, implies $\|A+n\|<(n+\lambda)\left(1+\left(\mu^{2} /(\lambda+n)^{2}\right)\right)^{1 / 2}$ for any real $\mu$, implies

$$
(\|A+n\|) /\left((n+\lambda)^{2}+\mu^{2}\right)^{1 / 2}<1,
$$

implies $(A+n)-(\lambda+i \mu+n)=A-(\lambda+i \mu)$ is invertible (with $\left\|(A-(\lambda+i \mu))^{-1}\right\|$ $\left.\leqq(\lambda-\rho(A))^{-1}\right)$, implies $\rho(A) \geqq \sup \operatorname{Re} \sigma(A)$.

Before we state the next proposition, let us note that if $A(\cdot) \in L^{1}(B(X),[a, b])$, $|\rho(A(\cdot))| \leqq\|A(\cdot)\|$, so that $\rho(A(\cdot)) \in L^{1}([a, b])$, so that we may consider

$$
\int_{a}^{t} \rho(A(s)) d s
$$

Proposition. $\|B(t, a)\| \leqq \exp \left(\int_{a}^{t} \rho(A(s)) d s\right)$.

Proof. Let $n>0$. Then $e^{n(t-a)} B(t, a)=\widetilde{B}(t, a)$ is absolutely continuous on $[a, b]$, $\widetilde{B}(a, a)=I$, and $\tilde{B}(\cdot, a) x$ is strongly differentiable a.e. $\forall x \in X$ with derivative $(A(\cdot)+n) \widetilde{B}(\cdot, a) x$. By the rough bound already obtained,

$$
\|\tilde{B}(t, a)\|=e^{n(t-a)}\|B(t, a)\| \leqq \exp \left(\int_{a}^{t}\|A(s)+n\| d s\right),
$$

so that

$$
\|B(t, a)\| \leqq \exp \left(\int_{a}^{t}(\|A(s)+n\|-n) d s\right) \quad \forall t \in[a, b]
$$

Since $|\|A(\cdot)+n\|-n| \leqq\|A(\cdot)\|$ and $(\|A(s)+n\|-n) \downarrow \rho(A(s)) \quad \forall s \in[a, b]$,

$$
\int_{a}^{t}(\|A(s)+n\|-n) d s \downarrow \int_{a}^{t} \rho(A(s)) d s,
$$

so that

$$
\|B(t, a)\| \leqq \exp \left(\int_{a}^{t} \rho(A(s)) d s\right) \quad \forall t \in[a, b]
$$

In general the bound for $\|B(t, a)\|$ given in the above proposition, though perhaps easy to work with, is by no means best possible. For example, if $A \in B(X)$ is nilpotent with $A^{n+1}=0$, then $\exp (t A)=1+t A+\cdots+\left(t^{n} / n !\right) A^{n}$, so that

$$
\|\exp (t A)\| \leqq 1+t\|A\|+\cdots+\left(t^{n} / n !\right)\|A\|^{n}=p(t),
$$

a polynomial. Since $\sigma(A)=0, \rho(A) \geqq 0$. The estimate given by the proposition in this case is $\|\exp (t A)\| \leqq e^{t \rho(A)}$. If $\rho(A)>0$, this is certainly not very good.

At least one amelioration of the estimate of the proposition is possible. Suppose $A \in B(X)$ is invertible. Then $A^{-1} B(\cdot, a) A$ is absolutely continuous on $[a, b]$, is the identity at $a$, and $A^{-1} B(\cdot, a) A x$ is strongly differentiable a.e. $\forall x \in X$ with derivative 
$A^{-1} A(\cdot) B(\cdot, a) A x=\left(A^{-1} A(\cdot) A\right)\left(A^{-1} B(\cdot, a) A\right) x$. By the estimate of the proposition $\left\|A^{-1} B(t, a) A\right\| \leqq \exp \left(\int_{a}^{t} \rho\left(A^{-1} A(s) A\right) d s\right)$. Thus

$$
\begin{aligned}
\|B(t, a)\|=\left\|A A^{-1} B(t, a) A A^{-1}\right\| & \leqq\left(\|A\| \cdot\left\|A^{-1}\right\|\right)\left\|A^{-1} B(t, a) A\right\| \\
& \leqq\left(\|A\| \cdot\left\|A^{-1}\right\|\right) \exp \left(\int_{a}^{t} \rho\left(A^{-1} A(s) A\right) d s\right),
\end{aligned}
$$

so that

$$
\|B(t, a)\| \leqq \inf _{\text {inverttble } A \in B(X)}\left(\|A\| \cdot\left\|A^{-1}\right\|\right) \exp \left(\int_{a}^{t} \rho\left(A^{-1} A(s) A\right) d s\right) .
$$

We shall give an example shortly of a nilpotent operator $A$ in Hilbert space for which this gives a fairly reasonable estimate of polynomial type on $\|\exp (t A)\|$. An estimate of this sort would seem to be of most value when

$$
A(\cdot) \in L^{1}(B(X),[a, b]) \quad \forall b>a
$$

and we wish to consider a bound on $\|B(t, a)\|$ as $t \rightarrow \infty$.

(c) Consider the function $\theta$ from $L^{1}(B(X),[a, b])$ into $L^{\infty}(B(X),[a, b])$ defined by $\theta: A(\cdot) \rightarrow B(\cdot, a)$. We know the range of $\theta$ consists of functions which are absolutely continuous on $[a, b]$, which are invertible everywhere on $[a, b]$, which are equal to $I$ at $a$, and which have $B(\cdot, a) x$ strongly differentiable $\forall x \in X$ with $B^{\prime}(\cdot, a) \in L^{1}(B(X),[a, b])$. In fact, the range of $\theta$ consists just of all such functions since $\theta\left(B^{\prime}(\cdot, a) B(\cdot, a)^{-1}\right)=B(\cdot, a)$ by uniqueness. Moreover, $\theta$ is $1-1$ since $\theta^{-1}(B(\cdot, a))=B^{\prime}(\cdot, a) B(\cdot, a)^{-1}$.

Let us investigate the continuity of $\theta$. We state two propositions.

Proposition. Let $A_{n}(\cdot)$ and $A(\cdot) \in L^{1}(B(X),[a, b])$, and let $A_{n}(\cdot) \rightarrow A(\cdot)$ in $L^{1}(B(X),[a, b])$. Then $B_{n}\left(\cdot, a_{0}\right) \rightarrow B\left(\cdot, a_{0}\right)$ in $L^{\infty}\left(B(X),\left[a_{0}, b\right]\right)$, uniformly over all $a_{0} \in[a, b]$.

Proof. Since $A_{n}(\cdot) \rightarrow A(\cdot)$ in $L^{1}(B(X),[a, b]), \exists k$ such that $\left\|A_{n}(\cdot)\right\|_{1}+\|A(\cdot)\|_{1}$ $\leqq k$. Since $\left\|B_{n}\left(t_{2}, t_{1}\right)\right\| \leqq \exp \left(\int_{t_{1}}^{t_{2}}\left\|A_{n}(s)\right\| d s\right)$ for $a \leqq t_{1} \leqq t_{2} \leqq b$, it follows that $\left\|B_{n}\left(t_{2}, t_{1}\right)\right\| \leqq e^{k}=K$, and, similarly, $\left\|B\left(t_{2}, t_{1}\right)\right\| \leqq K$.

We know that $\forall x \in X, B_{n}(t, s) B\left(s, a_{0}\right) x$ is absolutely continuous and differentiable a.e. in $s$ with derivative $B_{n}(t, s)\left(A(s)-A_{n}(s)\right) B\left(s, a_{0}\right) x$, so that

$$
\left(B\left(t, a_{0}\right)-B_{n}\left(t, a_{0}\right)\right) x=\int_{a_{0}}^{t} B_{n}(t, s)\left(A(s)-A_{n}(s)\right) B\left(s, a_{0}\right) x d s,
$$

implying

$$
\begin{aligned}
\left\|\left(B\left(t, a_{0}\right)-B_{n}\left(t, a_{0}\right)\right) x\right\| & \leqq\|x\| K^{2} \int_{a_{0}}^{t}\left\|A(s)-A_{n}(s)\right\| d s \\
& \leqq\|x\| K^{2} \int_{a}^{b}\left\|A(s)-A_{n}(s)\right\| d s=\|x\| K^{2}\left\|A(\cdot)-A_{n}(\cdot)\right\|_{1},
\end{aligned}
$$


so that

$$
\left\|B\left(\cdot, a_{0}\right)-B_{n}\left(\cdot, a_{0}\right)\right\|_{\infty} \leqq K^{2}\left\|A(\cdot)-A_{n}(\cdot)\right\|_{1},
$$

and $B_{n}\left(\cdot, a_{0}\right) \rightarrow B\left(\cdot, a_{0}\right)$ in $L^{\infty}\left(B(X),\left[a_{0}, b\right]\right)$, uniformly over $a_{0} \in[a, b]$.

Proposition. Let $A_{n}(\cdot)$ and $A(\cdot) \in L^{1}(B(X),[a, b])$, and let $A_{n}(\cdot) x \rightarrow A(\cdot) x$ in $L^{1}(X,[a, b]) \quad \forall x \in X$. Suppose further that $\exists k>0$ such that $\left\|A_{n}(\cdot)\right\|_{1}+\|A(\cdot)\|_{1} \leqq k$. Then $B_{n}\left(\cdot, a_{0}\right) x \rightarrow B\left(\cdot, a_{0}\right) x$ in $L^{\infty}\left(X,\left[a_{0}, b\right]\right) \quad \forall x \in X$, uniformly in $a_{0}$.

Proof. As before, $\left\|B_{n}\left(t_{2}, t_{1}\right)\right\| \leqq K=e^{k}$, and $\left\|B\left(t_{2}, t_{1}\right)\right\| \leqq K$ for $a \leqq t_{1} \leqq t_{2} \leqq b$.

If $x(\cdot)$ is any finite Riemann sum $\in L^{\infty}(X,[a, b])$, i.e., if

$$
\left.x(\cdot)=\sum_{i=1}^{n} x_{i} \chi_{\left[t_{1}-1\right.} t_{i}\right](\cdot) \quad\left(a=t_{0} \leqq \cdots \leqq t_{n}=b\right),
$$

then $A_{n}(\cdot) x(\cdot) \rightarrow A(\cdot) x(\cdot)$ in $L^{1}(X,[a, b])$.

Let $x \in X$, and let $\left\{x_{m}(\cdot)\right\}$ be a sequence of finite Riemann sums in $L^{\infty}(X,[a, b])$ such that $\left\|B(\cdot, a) x-x_{m}(\cdot)\right\| \leqq 1 / m$. Then as in the previous proposition, for $t \in[a, b]$,

$$
\left(B\left(t, a_{0}\right)-B_{n}\left(t, a_{0}\right)\right) x=\int_{a_{0}}^{t} B_{n}(t, s)\left(A(s)-A_{n}(s)\right) B\left(s, a_{0}\right) x d s,
$$

so that

$$
\begin{aligned}
\|\left(B\left(t, a_{0}\right)-\right. & \left.B_{n}\left(t, a_{0}\right)\right) x\left\|\leqq K \int_{a}^{t}\right\| A(s)-A_{n}(s)\|\cdot\| B(s, a) x-x_{m}(s) \| d s \\
& +K \int_{a}^{t}\left\|\left(A(s)-A_{n}(s)\right) x_{m}(s)\right\| d s \leqq(k K) / m+K\left\|\left(A(\cdot)-A_{n}(\cdot)\right) x_{m}(\cdot)\right\|_{1},
\end{aligned}
$$

so that $\left\|\left(B\left(\cdot, a_{0}\right)-B_{n}\left(\cdot, a_{0}\right)\right) x\right\| \leqq(k K) / m+K\left\|\left(A(\cdot)-A_{n}(\cdot)\right) x_{m}(\cdot)\right\|_{1}$, and

$$
B_{n}\left(\cdot, a_{0}\right) x \rightarrow B\left(\cdot, a_{0}\right) x
$$

in $L^{\infty}\left(X,\left[a_{0}, b\right]\right)$ by first choosing $m$ large and then letting $n \rightarrow \infty$, convergence being uniform over $a_{0} \in[a, b]$.

(d) When $X$ is a Hilbert space, some further statements can be made.

Note that if $A(\cdot) \in L^{1}(B(X),[a, b])$,

$$
\left(\int_{a}^{t} A(s) x d s, y\right)=\int_{a}^{t}(A(s) x, y) d s=\int_{a}^{t}\left(x, A(s)^{*} y\right) d s=\left(x, \int_{a}^{t} A(s)^{*} y d s\right),
$$

so that the operators $C(t)$ and $D(t)$ defined by $C(t) x=\int_{a}^{t} A(s) x d s$, and $D(t) x$ $=\int_{a}^{t} A(s)^{*} x d s$, are adjoints.

Thus if we set $B_{1}(\cdot, a)=\theta\left(-A(\cdot)^{*}\right), B_{1}(t, a)^{*} x=-\int_{a}^{t} B_{1}(s, a)^{*} A(s) x d s$, so that $B_{1}(t, a)^{*}=B(\cdot, a)^{-1}$. For example, if $A(t)$ is skew-adjoint $\forall t \in[a, b], B_{1}(\cdot, a)$ $=\theta(A(\cdot))=B(\cdot, a)$, so that $B(\cdot, a)^{*}=B(\cdot, a)^{-1}$ and $B(t, a)$ is unitary $\forall t \in[a, b]$.

Let $T=A+i B$, where $A$ and $B$ are self-adjoint. Suppose $M>\rho(T)$. Then $\exists n_{0}>0$ 
such that $\|A+i B+n\|-n<M$, or $\|A+i B+n\|<M+n$, for $n>n_{0}$. If $x$ is any unit vector,

$$
((A+i B+n) x,(A+i B+n) x)<M^{2}+n^{2}+2 M n,
$$

so that

$$
(1 / 2 n)\left(\left(\|A x\|^{2}+\|B x\|^{2}+2 \operatorname{Im}(A x, B x)-M^{2}\right)\right)+(A x, x)<M .
$$

The expression in the double parenthesis is majorized in absolute value by some $K>0$ independent of $x$, so that $(A x, x)<M+(K / 2 n) \quad \forall n>n_{0}$, and $(A x, x) \leqq M$ for all unit vectors $x$. Thus sup $\sigma(A) \leqq M$, and sup $\sigma(A) \leqq \rho(T)$.

If $M \leqq \rho(T)$, then the same argument carries through with > replaced everywhere by $\leqq$ and with $K / 2 n$ appearing on the left. Thus sup $\sigma(\grave{A}) \geqq \rho(T)$, and $\rho(T)=\sup \sigma(A)$.

[We digress briefly to state some pleasant consequences of the fact just proved.

(1) If $T$ has pure imaginary spectrum, then $\left(T+T^{*}\right)$ can be neither strictly positive nor strictly negative.

To show this we need only show that $\left(T+T^{*}\right)$ cannot be strictly negative, since $-T$ also has pure imaginary spectrum. But $\rho(T) \geqq \sup \operatorname{Re} \sigma(T)=0$, so that $\sup \sigma\left(\left(T+T^{*}\right) / 2\right) \geqq 0$.

(2) If $A$ is self-adjoint and $S$ is invertible, sup $\sigma\left(\operatorname{Re} S A S^{-1}\right)$ is minimal for $S=I$.

To see this we need only notice that $S A S^{-1}$ has the same spectrum as $A$, so that $\rho\left(S A S^{-1}\right) \geqq \rho(A)=\sup \sigma(A)$.

If $A$ is skew-adjoint, $\sigma\left(S A S^{-1}\right)$ is pure imaginary so that $\rho\left(S A S^{-1}\right) \geqq 0=\rho(A)$.]

From the digression it is clear that the amelioration of the estimate $\|B(t, a)\|$ $\leqq \exp \left(\int_{a}^{t} \rho(A(s)) d s\right)$ is no amelioration at all if $A(t)$ is either self-adjoint or skewadjoint for each $t$. Consider, however, the following example.

Let $X=L^{2}([0,1])$, and let $A f$ for $f \in X$ be defined by $(A f)(x)=f\left(x-\frac{1}{2}\right)$ for $x>\frac{1}{2},=0$ for $x<\frac{1}{2}$. Then $A^{2}=0$. Since $\left(A^{*} f\right)(x)=f\left(x+\frac{1}{2}\right)$ for $x<\frac{1}{2},=0$ for $x>\frac{1}{2}$, $\left(A+A^{*}\right)$ simply permutes the sections of a function on $\left[0, \frac{1}{2}\right]$ and $\left[\frac{1}{2}, 1\right]$, so that $\left\|A+A^{*}\right\|=1$. But $\left(A+A^{*}\right)(1)=1(1=$ the function identically equal to 1 on $[0,1])$, so that $\sup \sigma\left(A+A^{*}\right)=1$, and $\rho(A)=\frac{1}{2}$. Then the estimate gives $\|\exp (t A)\| \leqq e^{t / 2}$.

We may, however, compute $\|\exp (t A)\|$ directly. Certainly $\exp (t A)=1+t A$, and $t-1 \leqq\|\exp (t A)\| \leqq t+1$. More precisely, let $h=h \chi_{[0,1 / 2]}+h \chi_{[1 / 2,1]}=f+g$ be a function of unit norm in $X$. Then

$$
\begin{aligned}
(\exp (t A) h, \exp (t A) h) & =\int_{0}^{1}\left(h(x)+t f\left(x-\frac{1}{2}\right) x_{[1 / 2,1]}(x)\right)\left(\bar{h}(x)+t \vec{f}\left(x-\frac{1}{2}\right) x_{[1 / 2,1]}(x)\right) d x \\
& =1+t^{2}\|f\|^{2}+2 t \operatorname{Re} \int_{1 / 2}^{1} f\left(x-\frac{1}{2}\right) \bar{g}(x) d x .
\end{aligned}
$$

This can only increase if we assume that $f$ and $g$ are $\geqq 0$. If we fix $f$, then $\|g\|$ $=\left(1-\|f\|^{2}\right)^{1 / 2}$. Thus by the Schwartz inequality we can choose $g$ to maximize 
$(\exp (t A) h, \exp (t A) h)$ to $1+2 t\|f\|\left(1-\|f\|^{2}\right)^{1 / 2}+t^{2}\|f\|^{2}$. By calculus we may maximize this to $1+(t / 2)\left(\left(t^{2}+4\right)^{1 / 2}+t\right)$.

Let us now consider the estimate

$$
\|\exp (t A)\| \leqq \operatorname{irfi}_{\text {invertible } S \in B(X)}\left(\|S\| \cdot\left\|S^{-1}\right\| \exp \int_{0}^{t} \rho\left(S^{-1} A S\right) d s\right) .
$$

We consider only invertible $S$ of the form $M_{a}=$ multiplication by $e^{a x}$ for real $a$. For $f \in L^{2}([0,1]),\left(A M_{a} f\right)=e^{-a / 2} M_{a} A f$, so that $M_{a}^{-1} A M_{a}=e^{-a / 2} A$, and $\rho\left(M_{a}^{-1} A M_{a}\right)$ $=e^{-a / 2} \rho(A)=\frac{1}{2} e^{-a / 2}$. We need consider only $M_{a}$ for $a \geqq 0$ if we wish to obtain an improvement of the original estimate. Clearly $\left\|M_{a}\right\|=e^{a}$, and $\left\|M_{a}^{-1}\right\|=1$. We must therefore minimize

$$
\left\|M_{a}\right\| \cdot\left\|M_{a}^{-1}\right\| \exp \int_{0}^{t} \rho\left(M_{a}^{-1} A M_{a}\right) d s=e^{a} e^{t\left(e^{-a / 2} / 2\right)}=\exp \left(a+t\left(e^{-a / 2} / 2\right)\right)
$$

for $a \geqq 0$. I.e., we must minimize $a+t\left(e^{-a / 2} / 2\right)$ for $a \geqq 0$. We have

$$
(d / d a)\left(a+t\left(e^{-a / 2} / 2\right)\right)=1-\left(t e^{-a / 2} / 2\right) / 4 .
$$

Since this is always nonnegative for $t \leqq 4(a \geqq 0)$, the expression is minimized by choosing $a=0$ for $t \leqq 4$. For $t>4$, the derivative is negative at $a=0$. We therefore set $1-(t / 4) e^{-a / 2}=0$ to get $e^{a}=t^{2} / 16$ and $e^{-a / 2}=4 / t$ at this point, so that the minimum of the expression over $a \geqq 0$ for $t>4$ is $e^{2} t^{2} / 16$. Hence the improved general estimate is at least as good as $\|\exp (t A)\| \leqq e^{t / 2}$ for $t \leqq 4, \leqq e^{2} t^{2} / 16$ for $t>4$. Though not ideal, it is certainly better than $\|\exp (t A)\| \leqq e^{t / 2}$ for all $t$.

3. The Cauchy problem for unbounded operators in a separable Banach space.

(a) Definition. $C D(X)=$ the set of closed, densely-defined operators in $X$. $L(X)=$ the set of operators in $B(X)$ which are 1-1 with dense range.

Let $A \in L(X)$. Then $A^{-1} \in C D(X)$. Under the norm $\|x\|_{R(A)}=\left\|A^{-1} x\right\|$, the range of $A=R(A)$ becomes a Banach space since $\left\{x_{n}\right\}$ Cauchy in $R(A)$ means that $\left\{A^{-1} x_{n}\right\}$ is Cauchy in $X$, so that $\exists y \in X$ with $A^{-1} x_{n} \rightarrow y$ in $X$, so that $x_{n} \rightarrow A y$ in $R(A)$.

For $T \in B(X)$ with $T(R(A)) \subset R(A), A^{-1} T A$ is bounded by the closed graph theorem because $T A$ is bounded and has range in the domain of $A^{-1}$. For such $T$,

$$
\begin{aligned}
\|T\|_{R(A)} & =\sup _{\|x\|_{R(A)=1}}\|T x\|_{R(A)}=\sup _{\|x\|_{R(A)}=1}\left\|A^{-1} T x\right\| \\
& =\sup _{\|x\|=1}\left\|A^{-1} T A x\right\|=\left\|A^{-1} T A\right\| .
\end{aligned}
$$

If we set $\rho_{R(A)}(T)=\sup _{\alpha \geqq 0}\left(\|T+\alpha\|_{R(A)}-\alpha\right)$, then

$$
\rho_{R(A)}(T)=\sup _{\alpha \geq 0}\left(\left\|A^{-1}(T+\alpha) A\right\|-\alpha\right)=\sup _{\alpha \geq 0}\left(\left\|A^{-1} T A+\alpha\right\|-\alpha\right)=\rho\left(A^{-1} T A\right) .
$$

Finally, if $X$ is reflexive and $x_{n} \rightarrow x$ with $\left\|A^{-1} x_{n}\right\| \leqq K \quad \forall n$, then $\left\{A^{-1} x_{n}\right\}$ is Cauchy on all elements of $X^{*}$ of the form $f \circ A$, so that $\left\{A^{-1} x_{n}\right\}$ is Cauchy on all $f \in X^{*}$, being uniformly bounded. By weak completeness of the closed sphere 
$\exists y \in X$ such that $A^{-1} x_{n} \rightarrow y$ weakly. Since the graph of $A^{-1}$ is weakly closed, $x$ is in the domain of $A^{-1}$, and $A^{-1} x=y$. Also $\|y\| \leqq K$ since the norm-closed sphere is weakly closed.

(b) Although reflexivity of $X$ seems to be required to prove much in the way of existence for solutions of the Cauchy problem when unbounded operators are involved, we may derive the essential uniqueness lemma without it.

LEMMA. Let $A(\cdot)$ be a function from $[a, b]$ to $C D(X)$. Suppose $A \in L(X)$ has range in the domain of $A(t) \quad \forall t \in[a, b]$, and $A(\cdot) A \in L^{1}(B(X),[a, b])$. Suppose further that there exists a sequence $\left\{A_{n}(\cdot)\right\} \subset L^{1}(B(X),[a, b])$ for which the associated $\left\{f \circ B_{n}(t, s)\right\}$ are uniformly bounded by a constant $K(f)$ for each $f$ in $a w^{*}$-dense subspace $Y^{*}$ of $X^{*}$ as $n=1,2, \ldots$ and $a \leqq s \leqq t \leqq b$, and for which the functions $\left\|A_{n}(\cdot) A\right\|$ are all dominated by some fixed function in $L^{1}([a, b])$. Suppose $A_{n}(t) A$ $\rightarrow A(t) A$ strongly for almost all $t \in[a, b]$.

Then if $x_{i}(\cdot), i=1,2$, is a function from $[a, b]$ into the range of $A$ which is absolutely continuous and strongly differentiable a.e. with derivative $A(\cdot) x_{i}(\cdot)$, and which has $A^{-1} x_{i}(\cdot) \in L^{\infty}(X,[a, b]), x_{1}(a)=x_{2}(a)$ entails $x_{1}(t)=x_{2}(t) \quad \forall t \in[a, b]$.

Proof. Let $x(\cdot)=x_{1}(\cdot)-x_{2}(\cdot)$. Then $x(\cdot)$ satisfies the same hypotheses as $x_{1}(\cdot)$ and $x_{2}(\cdot)$, and, in addition, $x(a)=0$.

For any $n, B_{n}(t, \cdot) x(\cdot)$ is absolutely continuous on $[a, b]$ and strongly differentiable a.e. with derivative $B_{n}(t, \cdot)\left(-A_{n}(\cdot)+A(\cdot)\right) x(\cdot)$, so that for any $f \in Y^{*}$,

$$
f\left(x(t)-B_{n}(t, a) x(a)\right)=f(x(t))=\int_{a}^{t} f\left(B_{n}(t, s)\left(-A_{n}(s) A+A(s) A\right) A^{-1} x(s)\right) d s,
$$

so that $|f(x(t))| \leqq K(f) \int_{a}^{t}\left\|\left(A(s) A-A_{n}(s) A\right) A^{-1} x(s)\right\| d s \rightarrow 0$ as $n \rightarrow \infty$ because the integrand converges to zero for each $s$ and is dominated by a fixed function in $L^{1}([a, b])$. Thus $x(t)=0 \quad \forall t \in[a, b]$, and $x_{1}(\cdot)=x_{2}(\cdot)$.

(c) From now on unless otherwise stated we shall assume that $X$ is reflexive.

Note that if $x(\cdot) \in \mathscr{M}(X,[a, b])$ and if all $x(t)$ for $t \in[a, b]$ are in the range of $A \in L(X), A^{-1} x(\cdot) \in \mathscr{M}(X,[a, b])$. For if $f \in X^{*}$, there exists a sequence $\left\{f_{n} \circ A\right\}$ with $f_{n} \circ A \rightarrow f$ in $X^{*}$, so that $\left(f_{n} \cdot A\right)\left(A^{-1} x(\cdot)\right)=f_{n}(x(\cdot)) \rightarrow f\left(A^{-1} x(\cdot)\right)$ pointwise, implying that $f\left(A^{-1} x(\cdot)\right)$ is measurable on $[a, b]$.

Our fundamental existence theorems follow.

TheOREM 1. Let $A(\cdot)$ be a function from $[a, b]$ into $C D(X)$. Let $A \in L(X)$ have range in the domain of $A(t) \forall t \in[a, b]$, and let $A(\cdot) A \in L^{1}(B(X),[a, b])$. Let $C_{1}$ and $C_{2} \in B(X), C_{2}$ with dense range. Suppose $\exists$ a sequence $\left\{A_{n}(\cdot)\right\} \subset L^{1}(B(X),[a, b])$ (with associated $\left\{B_{n}(t, s)\right\}_{a \leq s \leq t \leq b}$ ) such that

(1) the range of $B_{n}(t, s) C_{1} C_{2}$ is in the domain of $A^{-1} \quad \forall n, a \leqq s \leqq t \leqq b$,

(2) $\exists K>0$ such that $\left\|B_{n}(t, s) C_{1}\right\|+\left\|A^{-1} B_{n}(t, s) C_{1} C_{2}\right\| \leqq K \quad \forall n, a \leqq s \leqq t \leqq b$,

(3) $\exists k(\cdot) \in L^{1}([a, b])$ such that $\left\|A_{n}(t) A\right\|+\|A(t) A\| \leqq k(t) \quad \forall t \in[a, b]$, and

(4) $\left(A_{n}(\cdot) A\right)^{*} f \rightarrow(A(\cdot) A)^{*} f$ strongly a.e. on $[a, b] \forall f \in X^{*}$. 
Then for $a \leqq s \leqq t \leqq b$, there exists $B_{C_{1} C_{2}}(t, s) \in B(X)$ bounded by $K$ and strongly continuous in $t$ for each such that

(1) $B_{C_{1} C_{2}}(t, t)=C_{1} \quad \forall t \in[a, b]$, and $B_{C_{1} C_{2}}(t, s) x=B_{C_{1} C_{2}}(t, s) y$ if $C_{1} x=C_{1} y$,

(2) $B_{C_{1} C_{2}}(t, s)$ carries the range of $C_{2}$ into the range of $A$, and

$$
\left\|A^{-1} B_{C_{1} C_{2}}(t, s) C_{2}\right\| \leqq K,
$$

(3) $B_{C_{1} C_{2}}(\cdot, s) C_{2}$ is absolutely continuous on $[s, b]$, and $B_{C_{1} C_{2}}(\cdot, s) C_{2} x$ is strongly differentiable a.e. on $[s, b] \quad \forall x \in X$ with derivative $A(\cdot) B_{C_{1} C_{2}}(\cdot, s) C_{2} x$, so that

$$
B_{C_{1} C_{2}}(t, s) C_{2} x=C_{1} C_{2} x+\int_{s}^{t} A(r) B_{C_{1} C_{2}}(r, s) C_{2} x d r \quad \forall x \in X
$$

Proof. Let $\left\{x_{j}\right\}$ and $\left\{f_{j}\right\}$ be sequences in $X$ and $X^{*}$, respectively, such that $\forall x \in X, f \in X^{*}, \varepsilon>0, \quad \exists j$ with $\left\|x-x_{j}\right\|<\varepsilon$ and $\left\|f-f_{j}\right\|<\varepsilon$. Fix $s$ and $t, a \leqq s \leqq t \leqq b$.

We have $\forall x \in X,\left(B_{n}(t, s)-I\right) C_{1} C_{2} x=\int_{s}^{t} A_{n}(r) B_{n}(r, s) C_{1} C_{2} x d r$, so that

$$
\begin{aligned}
\left|f\left(\left(B_{n}(t, s)-I\right)\right) C_{1} C_{2} x\right| & \leqq \int_{s}^{t}\left|f\left(A_{n}(r) A A^{-1} B_{n}(r, s) C_{1} C_{2} x\right)\right| d r \\
& \leqq K\|f\| \cdot\|x\| \int_{s}^{t} k(r) d r .
\end{aligned}
$$

Choose a subsequence $N_{1}$ of the integers such that $f_{1}\left(\left(B_{N_{1}(n)}(t, s)-I\right) C_{1} C_{2} x_{1}\right)$ converges to some value $B\left(t, s, f_{1}, x_{1}\right)$. We have

$$
\left|B\left(t, s, f_{1}, x_{1}\right)\right| \leqq K\left\|f_{1}\right\| \cdot\left\|x_{1}\right\| \int_{s}^{t} k(r) d r .
$$

Choose a subsequence $N_{2}$ of $N_{1}$ such that $f_{2}\left(\left(B_{N_{2}\left(N_{1}(n)\right)}(t, s)-I\right) C_{1} C_{2} x_{2}\right)$ converges to some value $B\left(t, s, f_{2}, x_{2}\right),\left|B\left(t, s, f_{2}, x_{2}\right)\right| \leqq K\left\|f_{2}\right\| \cdot\left\|x_{2}\right\| \int_{s}^{t} k(r) d r$. Continue, choosing a subsequence $N_{j}$ of $N_{j-1} \circ \cdots \circ N_{1}$ for which $f_{j}\left(\left(B_{N_{j} \circ \cdots \circ N_{1}(n)}(t, s)-I\right) C_{1} C_{2} x_{j}\right)$ converges to some value $B\left(t, s, f_{j}, x_{j}\right), \quad\left|B\left(t, s, f_{j}, x_{j}\right)\right| \leqq K\left\|f_{j}\right\| \cdot\left\|x_{j}\right\| \int_{s}^{t} k(r) d r$. Relabel, calling the diagonal sequence $=\left\{N_{n} \circ \cdots \circ N_{1}(n)\right\}$ simply $\{n\}$.

Then for each $j, f_{j}\left(\left(B_{n}(t, s)-I\right) C_{1} C_{2} x_{j}\right) \rightarrow B\left(t, s, f_{j}, x_{j}\right)$. Let $x \in X, f \in X^{*}$. Then $f\left(\left(B_{n}(t, s)-I\right) C_{1} C_{2} x\right)$ is Cauchy. For given $\varepsilon>0$, choose $x_{j} \in X$ and $f_{j} \in X^{*}$ such that $\left\|f-f_{j}\right\|<\min \left\{\|f\|,\left(6 K\left\|C_{2}\right\| \cdot\|x\|\right)^{-1} \varepsilon\right\}$, and $\left\|x-x_{j}\right\|<\varepsilon\left(12 K\left\|C_{2}\right\| \cdot\|f\|\right)^{-1}$ Then

$$
\begin{aligned}
\left|f\left(\left(B_{n}(t, s)-B_{m}(t, s)\right) C_{1} C_{2} x\right)\right| \leqq & \left|\left(f-f_{j}\right)\left(\left(B_{n}(t, s)-B_{m}(t, s)\right) C_{1} C_{2} x\right)\right| \\
& +\left|f_{j}\left(\left(B_{n}(t, s)-B_{m}(t, s)\right) C_{1} C_{2}\left(x-x_{j}\right)\right)\right| \\
& +\left|f_{j}\left(\left(B_{n}(t, s)-B_{m}(t, s)\right) C_{1} C_{2} x_{j}\right)\right| \\
< & (2 / 3) \varepsilon+\left|f_{f}\left(\left(B_{n}(t, s)-B_{m}(t, s)\right) C_{1} C_{2} x_{j}\right)\right| .
\end{aligned}
$$

We have only to choose $n_{0}$ such that $m, n>n_{0}$ makes the last term $<\varepsilon / 3$.

Call the limit of $f\left(\left(B_{n}(t, s)-I\right) C_{1} C_{2} x\right), B(t, s, f, x)$. 
Now let $\left\{t_{j}\right\}$ be a sequence dense in $[s, b]$. Again by using the diagonal process and ultimately relabeling the final subsequence, we may assume that $f\left(\left(B_{n}\left(t_{j}, s\right)-I\right) C_{1} C_{2} x\right)$ converges to some $B\left(t_{j}, s, f, x\right) \quad \forall x \in X, f \in X^{*}$. Then $f\left(\left(B_{n}(t, s)-I\right) C_{1} C_{2} x\right)$ converges to some $B(t, s, f, x) \quad \forall x \in X, f \in X^{*}, t \in[s, b]$. For, taking $s \leqq t_{j} \leqq t$, we have

$$
\begin{aligned}
& \left|f\left(\left(B_{n}(t, s)-I\right) C_{1} C_{2} x\right)-f\left(\left(B_{m}(t, s)-I\right) C_{1} C_{2} x\right)\right| \\
& \leqq \\
& \quad+\left|f\left(\left(B_{n}(t, s)-B_{n}\left(t_{j}, s\right)\right) C_{1} C_{2} x\right)\right|+\left|f\left(\left(B_{m}(t, s)-B_{m}\left(t_{j}, s\right)\right) C_{1} C_{2} x\right)\right| \\
& \quad+\left|f\left(\left(B_{m}\left(t_{j}, s\right)-B_{n}\left(t_{j}, s\right)\right) C_{1} C_{2} x\right)\right| .
\end{aligned}
$$

The last term can be made arbitrarily small for fixed $j$ by taking $m$ and $n$ suitably large. Since

$$
\begin{aligned}
\left|f\left(\left(B_{n}(t, s)-B_{n}\left(t_{j}, s\right)\right) C_{1} C_{2} x\right)\right| & \leqq \int_{t_{j}}^{t}\left|f\left(A_{n}(r) A A^{-1} B_{n}(r, s) C_{1} C_{2} x\right)\right| d r \\
& \leqq K\|f\| \cdot\|x\| \int_{t_{j}}^{t} k(r) d r
\end{aligned}
$$

the first two terms can be made arbitrarily small by choosing $t$, sufficiently close to $t$.

Consider now $f\left(\left(B_{n}(t, s)-I\right) C_{1} x\right)$. This is Cauchy $\forall x \in X, f \in X^{*}, t \in[s, b]$, because $f \circ\left(B_{n}(t, s)-I\right) C_{1}$ is a bounded sequence of linear functionals (bounded by $\left.\|f\|\left(K+\left\|C_{1}\right\|\right)\right)$ converging pointwise on a dense subset of $X$, namely, the range of $C_{2}$.

Thus $f\left(B_{n}(t, s) C_{1} x\right) \rightarrow$ some $f\left(B_{C_{1} C_{2}}(t, s) x\right)$. This expression is clearly linear in $f$ and $x$ since each of the convergents is, and since each $f\left(B_{n}(t, s) C_{1} x\right)$ is bounded by $K\|f\| \cdot\|x\|,\left|f\left(B_{C_{1} C_{2}}(t, s) x\right)\right| \leqq K\|f\| \cdot\|x\|$. By reflexivity of $X, B_{C_{1} C_{2}}(t, s) \in B(X)$ with $\left\|B_{C_{1} C_{2}}(t, s)\right\| \leqq K$. Since each $B_{n}(s, s)=I, B_{C_{1} C_{2}}(s, s)=C_{1}$. If we anticipate the remainder of this proof by assuming that $B_{C_{1} C_{2}}(\cdot, s) C_{2}$ is absolutely continuous on $[s, b]$ (or even only continuous), then $B_{C_{1} C_{2}}(\cdot, s)$ is strongly continuous on $[s, b]$ since bounded strong convergence on the dense subset consisting of the range of $C_{2}$ implies strong convergence on all of $X$. Clearly $B_{C_{1} C_{2}}(t, s) x=0$ if $C_{1} x=0$.

We have now that each $f\left(B_{C_{1} C_{2}}(\cdot, s) x\right)$ is a pointwise limit on $[s, b]$ of the measurable (indeed, continuous) functions $f\left(B_{n}(\cdot, s) C_{1} x\right)$. Hence $B_{C_{1} C_{2}}(\cdot, s) \in$ $L^{\infty}(B(X),[s, b])$ with $\left\|B_{C_{1} C_{2}}(\cdot, s)\right\|_{\infty} \leqq K$, and $B_{C_{1} C_{2}}(\cdot, s) C_{2} \in L^{\infty}(B(X),[s, b])$ with $\left\|B_{C_{1} C_{2}}(\cdot, s) C_{2}\right\| \leqq K\left\|C_{2}\right\|$. By an earlier remark, $B_{C_{1} C_{2}}(t, s) C_{2} x$ is in the domain of $A^{-1} \quad \forall x \in X, t \in[s, b]$, and $\left\|A^{-1} B_{C_{1} C_{2}}(t, s) C_{2} x\right\| \leqq K\|x\|$. I.e., $A^{-1} B_{C_{1} C_{2}}(\cdot, s) C_{2}$ belongs to $L^{\infty}(B(X),[s, b])$ with $\left\|A^{-1} B_{C_{1} C_{2}}(\cdot, s) C_{2}\right\|_{\infty} \leqq K\left(A^{-1} B_{C_{1} C_{2}}(\cdot, s) C_{2}\right.$ is measurable by the remark immediately preceding this theorem).

Let us show that $\forall x \in X, f \in X^{*}, t \in[s, b]$,

$$
f\left(B_{C_{1} C_{2}}(t, s) C_{2} x\right)=f\left(C_{1} C_{2} x\right)+\int_{s}^{t} f\left((A(r) A)\left(A^{-1} B_{C_{1} C_{2}}(r, s) C_{2}\right) x\right) d r
$$


We have

$$
\begin{aligned}
& \mid f\left(C_{1} C_{2} x\right)+\int_{s}^{t} f\left((A(r) A)\left(A^{-1} B_{C_{1} C_{2}}(r, s) C_{2} x\right) d r-f\left(B_{n}(t, s) C_{1} C_{2} x\right) \mid\right. \\
& \leqq \\
& \int_{s}^{t}\left|f\left(\left(A(r) A-A_{n}(r) A\right)\left(A^{-1} B_{n}(r, s) C_{1} C_{2}\right) x\right)\right| d r \\
& \quad+\int_{s}^{t}\left|f\left((A(r) A)\left(\left(A^{-1} B_{n}(r, s) C_{1} C_{2}\right)-\left(A^{-1} B_{C_{1} C_{2}}(r, s) C_{2}\right)\right) x\right)\right| d r .
\end{aligned}
$$

The integrand of the first term is dominated by $K\|f\| \cdot\|x\| k(r)$, and it goes to zero pointwise a.e. on $[s, t]$ since $\left(A_{n}(r) A\right)^{*} f \rightarrow(A(r) A)^{*} f$ strongly for almost all $r \in[s, b]$, and $\left\|A^{-1} B_{n}(r, s) C_{1} C_{2} x\right\| \leqq K\|x\| \quad \forall r \in[s, b]$. Thus the first term goes to zero as $n \rightarrow \infty$. The integrand of the second term is similarly dominated, and

$$
f \circ\left((A(r) A)\left(A^{-1} B_{n}(r, s) C_{1} C_{2}-A^{-1} B_{C_{1} C_{2}}(r, s) C_{2}\right) x\right) \rightarrow 0
$$

as $n \rightarrow \infty$ since for fixed $r$ and $s, A^{-1} B_{n}(r, s) C_{1} C_{2} \rightarrow A^{-1} B_{C_{1} C_{2}}(r, s) C_{2}$ weakly. Thus the second term goes to zero. I.e., $f\left(B_{n}(t, s) C_{1} C_{2} x\right)$ converges to the right side of the desired equality. But it also converges to $f\left(B_{C_{1} C_{2}}(t, s) C_{2} x\right)$, so that

$$
B_{C_{1} C_{2}}(t, s) C_{2} x=C_{1} C_{2} x+\int_{s}^{t} A(r) B_{C_{1} C_{2}}(r, s) C_{2} x d r \quad \forall t \in[s, b]
$$

the integrand being in $L^{1}(B(X),[s, b])$.

All remaining conclusions of the theorem now follow directly.

THEOREM 2. Suppose in addition to the hypothesis of Theorem 1 that $A_{n}(\cdot) A x$ $\rightarrow A(\cdot) A x$ strongly a.e. on $[a, b]$ for each $x \in X$, and that $\exists C_{3} \in B(X)$ such that $C_{3}$ is 1-1 and $\exists K^{\prime}>0$ with $\left\|C_{3} B_{n}(t, s)\right\| \leqq K^{\prime} \quad \forall n, a \leqq s \leqq t \leqq b$. Then if $x(\cdot) \in L^{\infty}(X,[s, b])$ is absolutely continuous with $x(t)$ in the domain of $A^{-1} \quad \forall t \in[s, b]$ in such wise that $A^{-1} x(\cdot) \in L^{\infty}(X,[s, b])$, and if $x(\cdot)$ is strongly differentiable a.e. on $[s, b]$ with derivative $A(\cdot) x(\cdot)$, then $x(s)=C_{1} C_{2} x$ entails $x(t)=B_{C_{1} C_{2}}(t, s) C_{2} x \quad \forall t \in[s, b]$.

Proof. Apply the uniqueness lemma to $x(\cdot)$ and to $B_{C_{1} C_{2}}(\cdot, s) C_{2} x$.

Corollary 1. If $C(\cdot) \in L^{\infty}(B(X),[s, b])$ is such that $C(s)=C_{1}, C(\cdot) C_{2}$ is absolutely continuous on $[s, b]$ with range in the domain of $A^{-1}$ in such wise that $A^{-1} C(\cdot) C_{2} \in L^{\infty}(B(X),[s, b])$, and if $C(\cdot) C_{2} x$ is strongly differentiable a.e. on $[s, b]$ with derivative $A(\cdot) C(\cdot) C_{2} x \quad \forall x \in X$, then $C(\cdot)=B_{C_{1} C_{2}}(\cdot, s)$.

Proof. By the theorem $C(\cdot) C_{2} x=B_{C_{1} C_{2}}(\cdot, s) C_{2} x \quad \forall x \in X$. Since the range of $C_{2}$ is dense in $X, C(\cdot)=B_{C_{1} C_{2}}(\cdot, s)$.

Corollary 2. Let $s_{0} \in[s, b]$. Let $x \in X$. If $B_{C_{1} C_{2}}\left(s_{0}, s\right) C_{2} x=C_{1} y$ for some $y \in X$, then $B_{C_{1} C_{2}}(t, s) C_{2} x=B_{C_{1} C_{2}}\left(t, s_{0}\right) C_{1}^{-1} B_{C_{1} C_{2}}\left(s_{0}, s\right) C_{2} x$ for all $t \in\left[s_{0}, b\right]$. If the range of $B_{C_{1} C_{2}}\left(s_{0}, s\right)$ lies in the range of $C_{1}$, then

$$
B_{C_{1} C_{2}}(t, s)=B_{C_{1} C_{2}}\left(t, s_{0}\right) C_{1}^{-1} B_{C_{1} C_{2}}\left(s_{0}, s\right) \quad \forall t \in\left[s_{0}, b\right] .
$$

Proof. Let $x(t)=B_{C_{1} C_{2}}(t, s) C_{2} x$ for $t \in\left[s, s_{0}\right],=B_{C_{1} C_{2}}\left(t, s_{0}\right) C_{1}^{-1} B_{C_{1} C_{2}}\left(s_{0}, s\right) C_{2} x$ for 
$t \in\left[s_{0}, b\right]$. Then $x(\cdot)$ satisfies the hypotheses of the theorem, so that $B_{C_{1} C_{2}}(t, s) C_{2} x$ $=x(t) \quad \forall t \in[s, b]$, in particular, for $t \in\left[s_{0}, b\right]$.

If the range of $B_{C_{1} C_{2}}\left(s_{0}, s\right)$ lies in the range of $C_{1}, B_{C_{1} C_{2}}\left(t, s_{0}\right) C_{1}^{-1} B_{C_{1} C_{2}}\left(s_{0}, s\right)$ is well-defined, and $B_{C_{1} C_{2}}(t, s) C_{2} x=B_{C_{1} C_{2}}\left(t, s_{0}\right) C_{1}^{-1} B_{C_{1} C_{2}}\left(s_{0}, s\right) C_{2} x$ by the first part of the corollary $\forall x \in X$, so that $B_{C_{1} C_{2}}(t, s)=B_{C_{1} C_{2}}\left(t, s_{0}\right) C_{1}^{-1} B_{C_{1} C_{2}}\left(s_{0}, s\right)$ for $t \in\left[s_{0}, b\right]$ by density of the range of $C_{2}$.

THEOREM 3. Suppose in addition to the hypotheses of Theorems 1 and 2 that $C_{1} \in L(X)$. Then $C_{3} B_{C_{1} C_{2}}(t, s) C_{1}^{-1} \in B(X), a \leqq s \leqq t \leqq b$, and $\left\|C_{3} B_{C_{1} C_{2}}(t, s) C_{1}^{-1}\right\| \leqq K^{\prime}$. Further, if $B_{n}(t, s) C_{1} \rightarrow B_{C_{1} C_{2}}(t, s)$ weakly, $C_{3} B_{n}(t, s) \rightarrow C_{3} B_{C_{1} C_{2}}(t, s) C_{1}^{-1}$ weakly. Also, $C_{3} B_{C_{1} C_{2}}(t, \cdot) C_{1}^{-1} A x$ is absolutely continuous on $[a, t]$, and $C_{3} B_{C_{1} C_{2}}(t, \cdot) C_{1}^{-1} A x$ is strongly differentiable a.e. on $[a, t]$ with derivative $-C_{3} B_{C_{1} C_{2}}(t, \cdot) C_{1}^{-1} A(\cdot) A x$.

Proof. Let us assume that $B_{n}(t, s) C_{1} \rightarrow B_{C_{1} C_{2}}(t, s)$ weakly. Then $C_{3} B_{n}(t, s) C_{1}$ $\rightarrow C_{3} B_{C_{1} C_{2}}(t, s)$ weakly. Let $y=C_{1} x,\|y\| \leqq 1$. We have

$$
C_{3} B_{n}(t, s) C_{1} x=C_{3} B_{n}(t, s) y \rightarrow C_{3} B_{C_{1} C_{2}}(t, s) x=C_{3} B_{C_{1} C_{2}}(t, s) C_{1}^{-1} y
$$

weakly. Then $\left\|C_{3} B_{C_{1} C_{2}}(t, s) C_{1}^{-1} y\right\| \leqq \lim \inf \left\|C_{3} B_{n}(t, s) y\right\| \leqq K^{\prime}$, so that

$$
\left\|C_{3} B_{C_{1} C_{2}}(t, s) C_{1}^{-1}\right\| \leqq K^{\prime} .
$$

Since $C_{3} B_{n}(t, s)$ is bounded by $K^{\prime} \quad \forall n$ and since $C_{3} B_{n}(t, s)$ converges weakly to $C_{3} B_{C_{1} C_{2}}(t, s) C_{1}^{-1}$ on the range of $C_{1}$, which is dense, $C_{3} B_{n}(t, s) \rightarrow C_{3} B_{C_{1} C_{2}}(t, s) C_{1}^{-1}$ weakly.

Let us show that

$$
f\left(C_{3} B_{C_{1} C_{2}}(t, s) C_{1}^{-1} A x\right)=f\left(C_{3} A x\right)+\int_{3}^{t} f\left(C_{3} B_{C_{1} C_{2}}(t, r) C_{1}^{-1} A(r) A x\right) d r .
$$

We have $B_{n}(t, s) x=x+\int_{s}^{t} B_{n}(t, r) A_{n}(r) x d r$, so that

$$
\begin{aligned}
&\left|f\left(C_{3} A x\right)+\int_{8}^{t} f\left(C_{3} B_{C_{1} C_{2}}(t, r) C_{1}^{-1} A(r) A x\right) d r-f\left(C_{3} B_{n}(t, s) A x\right)\right| \\
& \leqq \int_{8}^{t}\left|f\left(C_{3} B_{n}(t, r)\left(A(r) A-A_{n}(r) A x\right)\right)\right| d r \\
& \quad+\int_{8}^{t}\left|f\left(\left(C_{3} B_{n}(t, r)-C_{3} B_{C_{1} C_{2}}(t, r) C_{1}^{-1}\right) A(r) A x\right)\right| d r .
\end{aligned}
$$

The integrand of the first integral is dominated by $K^{\prime}\|f\| \cdot\|x\| k(r)$, and since $A_{n}(r) A \rightarrow A(r) A$ strongly a.e., the integrand goes to zero pointwise as $n \rightarrow \infty$. Thus the integral goes to zero as $n \rightarrow \infty$. The integrand of the second integral is dominated by $2 K^{\prime}\|f\| \cdot\|x\| k(r)$, and since $C_{3} B_{n}(t, r) \rightarrow C_{3} B_{C_{1} C_{2}}(t, r) C_{1}^{-1}$ weakly for fixed $t$ and $r$, the integrand goes pointwise to zero as $n \rightarrow \infty$, and hence this integral, too, goes to zero as $n \rightarrow \infty$. But $f\left(C_{3} B_{n}(t, s) A x\right) \rightarrow f\left(C_{3} B_{C_{1} C_{2}}(t, s) C_{1}^{-1} A x\right)$, so that $C_{3} B_{C_{1} C_{2}}(t, s) C_{1}^{-1} A x=C_{3} A x+\int_{s}^{t} C_{3} B_{C_{1} C_{2}}(t, r) C_{1}^{-1} A(r) A x d r$, the integrand of the integral being in $L^{1}(B(X),[s, t])$.

The remaining conclusions of the theorem are now immediate. 
At least one generalization of these theorems is possible. Suppose $U(\cdot) \in$ $L^{\infty}(B(X),[a, b])$ is absolutely continuous, and suppose that $U(\cdot) x$ is strongly differentiable a.e. $\forall x \in X$ with derivative $U^{\prime}(\cdot) x$, where $U^{\prime}(\cdot) \in L^{1}(B(X),[a, b])$. If, in addition, each $U(t)$ is invertible, we may write $U^{\prime}(\cdot)=\left(U^{\prime}(\cdot) U(\cdot)^{-1}\right) U(\cdot)$, where $U^{\prime}(\cdot) U(\cdot)^{-1} \in L^{1}(B(X),[a, b])$ by continuity, and, hence, boundedness of $U(\cdot)^{-1}$. Then, since $U(\cdot) U(a)^{-1}$ is the identity at $a, U(t) U(a)^{-1} x=x+$ $\int_{a}^{t}\left(U^{\prime}(s) U(s)^{-1}\right)\left(U(s) U(a)^{-1}\right) x d s$, and, consequently,

$$
U(a) U(t)^{-1} x=x-\int_{a}^{t}\left(U(a) U(s)^{-1}\right)\left(U^{\prime}(s) U(s)^{-1}\right) x d s
$$

$\forall x \in X$ by the fundamental propositions for bounded operators. Thus $U(\cdot)^{-1}$ is absolutely continuous, and $U(\cdot)^{-1} x$ is strongly differentiable a.e. $\forall x \in X$ with derivative $-U(\cdot)^{-1} U^{\prime}(\cdot) U(\cdot)^{-1} x$.

Having made these remarks, we may now generalize the three above theorems as follows.

TheOREM 1'. Let $U(\cdot) \in L^{\infty}(B(X),[a, b])$ be absolutely continuous, and let $U(t)$ be invertible $\forall t \in[a, b]$. Set $K_{0}$ equal to $\|U(\cdot)\|_{\infty}\left\|U(\cdot)^{-1}\right\|_{\infty}$. Let $U(\cdot) x$ be strongly differentiable a.e. $\forall x \in X$ with derivative $U^{\prime}(\cdot) x$, where

$$
U^{\prime}(\cdot) \in L^{1}(B(X),[a, b]) \text {. }
$$

Let $\bar{A}(\cdot)$ be a function from $[a, b]$ to $C D(X)$ such that $A(\cdot)=-U(\cdot)^{-1} U^{\prime}(\cdot)$ $+U(\cdot)^{-1} \bar{A}(\cdot) U(\cdot)$ satisfies the hypotheses of Theorem 1 with $A, C_{1}, C_{2}$, and $K$ as given in that theorem. Set $C_{1}(t)=U(t) C_{1} U(t)^{-1}, C_{2}(t)=U(t) C_{2} U(t)^{-1}$, and $P(t)=U(t) A U(t)^{-1} \quad \forall t \in[a, b]$. (Clearly all of $C_{1}(\cdot), C_{2}(\cdot)$, and $P(\cdot)$ are absolutely continuous on $[a, b]$ and strongly differentiable a.e. with the obvious derivatives. The range of $C_{2}(t)$ is dense in $X \quad \forall t \in[a, b]$, and $P(t) \in L(X) \quad \forall t \in[a, b]$.)

Then $\bar{A}(\cdot) P(\cdot) \in L^{1}(B(X),[a, b])$, and for $a \leqq s \leqq t \leqq b, \quad \exists \bar{B}_{C_{1} C_{2}}(t, s) \in B(X)$ bounded by $K K_{0}$ and strongly continuous in $t$ for each such that

(1) $\bar{B}_{C_{1} C_{2}}(t, t)=C_{1}(t) \quad \forall t \in[a, b]$, and $\bar{B}_{C_{1} C_{2}}(t, s) x=\bar{B}_{C_{1} C_{2}}(t, s) y$ if $C_{1}(s) x=C_{1}(s) y$,

(2) $\bar{B}_{C_{1} C_{2}}(t, s)$ carries the range of $C_{2}(s)$ into the domain of $P(t)^{-1}$, and

$$
\left\|P(t)^{-1} \bar{B}_{C_{1} C_{2}}(t, s) C_{2}(s)\right\| \leqq K K_{0},
$$

(3) $\bar{B}_{C_{1} C_{2}}(\cdot, s) C_{2}(s)$ is absolutely continuous on $[s, b]$ and $\bar{B}_{C_{1} C_{2}}(\cdot, s) C_{2}(s) x$ is strongly differentiable a.e. on $[s, b] \quad \forall x \in X$ with derivative $\bar{A}(\cdot) \bar{B}_{C_{1} C_{2}}(\cdot, s) C_{2}(s) x$, so that $\bar{B}_{C_{1} C_{2}}(t, s) C_{2}(s) x=C_{1}(s) C_{2}(s) x+\int_{s}^{t} \bar{A}(r) \bar{B}_{C_{1} C_{2}}(r, s) C_{2}(s) x d r \quad \forall x \in X$.

Proof. Since $A(\cdot) A \in L^{1}(B(X),[a, b]), A(\cdot) A+U(\cdot)^{-1} U^{\prime}(\cdot) A$ belongs to $L^{1}(B(X),[a, b])$, and, consequently, $\bar{A}(\cdot) U(\cdot) A U(\cdot)^{-1}=\bar{A}(\cdot) P(\cdot)$ belongs to $L^{1}(B(X),[a, b])$. Let $B_{C_{1} C_{2}}(t, s)$ be as in Theorem 1 , and set $\bar{B}_{C_{1} C_{2}}(t, s)=$ $U(t) B_{C_{1} C_{2}}(t, s) U(s)^{-1}$. Then $\left\|\bar{B}_{C_{1} C_{2}}(t, s)\right\| \leqq K K_{0}$. By strong continuity and boundedness of $B_{C_{1} C_{2}}(t, s)$ in $t$, and by uniform continuity of $U(t), \bar{B}_{C_{1} C_{2}}(t, s)$ is strongly continuous in $t$. 
We have $\bar{B}_{C_{1} C_{2}}(t, t)=U(t) B_{C_{1} C_{2}}(t, t) U(t)^{-1}=C_{1}(t) \quad \forall t \in[a, b]$. If $C_{1}(s) x=$ $C_{1}(s) y$, then $C_{1} U(s)^{-1} x=C_{1} U(s)^{-1} y$, so that

$$
B_{C_{1} C_{2}}(t, s) U(s)^{-1} x=B_{C_{1} C_{2}}(t, s) U(s)^{-1} y,
$$

and $\bar{B}_{C_{1} C_{2}}(t, s) x=\bar{B}_{C_{1} C_{2}}(t, s) y$.

The range of $\bar{B}_{C_{1} C_{2}}(t, s) C_{2}(s)$ is the range of $U(t) B_{C_{1} C_{2}}(t, s) U(s)^{-1} U(s) C_{2} U(s)^{-1}$, which has the same range as $U(t) B_{C_{1} \mathrm{C}_{2}}(t, s) C_{2}$, which has range in the range of $U(t) A$, which lies in the domain of $P(t)^{-1}=U(t) A^{-1} U(t)^{-1}$. We have

$$
\left\|P(t)^{-1} \bar{B}_{C_{1} C_{2}}(t, s) C_{2}(s)\right\|=\left\|U(t) A^{-1} B_{C_{1} C_{2}}(t, s) C_{2} U(s)^{-1}\right\| \leqq K K_{0} .
$$

Since $\bar{B}_{C_{1} C_{2}}(\cdot, s) C_{2}(s)=U(\cdot)\left(B_{C_{1} C_{2}}(\cdot, s) C_{2}\right) U(s)^{-1}$, it is absolutely continuous on $[a, b]$, and it is strongly differentiable a.e. on all $x \in X$ with derivative

$$
\begin{aligned}
U^{\prime}(\cdot)\left(B_{C_{1} C_{2}}(\cdot, s) C_{2}\right) U(s)^{-1}+U(\cdot)\left(-U(\cdot)^{-1} U^{\prime}(\cdot)+U(\cdot)^{-1} \bar{A}(\cdot) U(\cdot)\right) \\
\cdot B_{C_{1} C_{2}}(\cdot, s) C_{2} U(s)^{-1}=\bar{A}(\cdot) \bar{B}_{C_{1} C_{2}}(\cdot, s) C_{2}(s) .
\end{aligned}
$$

THEOREM 2'. Suppose in addition to the hypotheses of Theorem $1^{\prime}$ the hypotheses of Theorem 2 hold for $A(\cdot)$. Then if $x(\cdot) \in L^{\infty}(X,[s, b])$ is absolutely continuous with $x(t)$ in the domain of $P(t)^{-1} \quad \forall t \in[s, b]$ in such wise that $P(t)^{-1} x(t) \in L^{\infty}(X,[s, b])$, and if $x(\cdot)$ is strongly differentiable a.e. on $[s, b]$ with derivative $\bar{A}(\cdot) x(\cdot)$, then $x(s)=C_{1}(s) C_{2}(s) x$ entails $x(t)=\bar{B}_{C_{1} C_{2}}(t, s) C_{2}(s) x \quad \forall t \in[s, b]$.

Proof. It suffices to show that $U(t)^{-1} x(t)=B_{C_{1} C_{2}}(t, s) C_{2} U(s)^{-1} x \quad \forall t \in[s, b]$. But $U(\cdot)^{-1} x(\cdot)$ is absolutely continuous, $U(t)^{-1} x(t)$ is in the domain of $A^{-1}$ because $x(t)$ is in the domain of $P(t)^{-1}=U(t) A^{-1} U(t)^{-1}$, and $A^{-1} U(\cdot)^{-1} x(\cdot)$ $=U(\cdot)^{-1} P(\cdot)^{-1} x(\cdot) \in L^{\infty}(X,[s, b])$. Further, $U(\cdot)^{-1} x(\cdot)$ is strongly differentiable a.e. on $[s, b]$ with derivative

$$
\begin{aligned}
U(\cdot)^{-1} x(\cdot) & +U(\cdot)^{-1} x^{\prime}(\cdot)=-U(\cdot)^{-1} U^{\prime}(\cdot) U(\cdot)^{-1} x(\cdot)+U(\cdot)^{-1} \bar{A}(\cdot) x(\cdot) \\
& =\left(-U(\cdot)^{-1} U^{\prime}(\cdot)+U(\cdot)^{-1} \bar{A}(\cdot) U(\cdot)\right) U(\cdot)^{-1} x(\cdot)=A(\cdot)\left(U(\cdot)^{-1} x(\cdot)\right) .
\end{aligned}
$$

Finally, $U(s)^{-1} x(s)=U(s)^{-1} C_{1}(s) C_{2}(s) x=C_{1} C_{2} U(s)^{-1} x$.

By Theorem 2, $U(t)^{-1} x(t)=B_{C_{1} C_{2}}(t, s) C_{2} U(s)^{-1} x \quad \forall t \in[a, b]$.

Corollary $1^{\prime}$. If $C(\cdot) \in L^{\infty}(B(X),[s, b])$ is such that $C(s)=C_{1}(s), C(\cdot) C_{2}(s)$ is absolutely continuous on $[s, b]$ with range in the domain of $P(t)^{-1}$ in such wise that $P(t)^{-1} C(\cdot) C_{2}(s)$ belongs to $L^{\infty}(B(X),[s, b])$, and if $C(\cdot) C_{2}(s) x$ is strongly differentiable a.e. on $[s, b]$ with derivative $\bar{A}(\cdot) C(\cdot) C_{2}(s) x \quad \forall x \in X$, then $C(\cdot)=\bar{B}_{C_{1} C_{2}}(\cdot, s)$.

Proof. By Theorem $2^{\prime}, C(\cdot) C_{2}(s) x=\bar{B}_{C_{1} C_{2}}(\cdot, s) C_{2}(s) x \quad \forall x \in X$. Since $C_{2}(s)$ has dense range, $C(\cdot)=\bar{B}_{C_{1} C_{2}}(\cdot, s)$.

Corollary $2^{\prime}$. Let $s_{0} \in[s, b]$, and let $x \in X$. If $\bar{B}_{C_{1} C_{2}}\left(s_{0}, s\right) C_{2}(s) x=C_{1}\left(s_{0}\right) y$ for some $y \in X$, then

$$
\bar{B}_{C_{1} C_{2}}(t, s) C_{2}(s) x=\bar{B}_{C_{1} C_{2}}\left(t, s_{0}\right) C_{1}\left(s_{0}\right)^{-1} \bar{B}_{C_{1} C_{2}}\left(s_{0}, s\right) C_{2}(s) x \quad \forall t \in\left[s_{0}, b\right]
$$


If the range of $\bar{B}_{C_{1} C_{2}}\left(s_{0}, s\right)$ lies in the range of $C_{1}\left(s_{0}\right)$, then

$$
\bar{B}_{C_{1} C_{2}}(t, s)=\bar{B}_{C_{1} C_{2}}\left(t, s_{0}\right) C_{1}\left(s_{0}\right)^{-1} \bar{B}_{C_{1} C_{2}}\left(s_{0}, s\right) \quad \forall t \in\left[s_{0}, b\right]
$$

Proof. Exactly as for Corollary 2.

THEOREM 3'. Suppose in addition to the hypotheses of Theorems 1' and 2', $C_{1}(t) \in L(X) \quad \forall t \in[a, b]$, i.e., $C_{1} \in L(X)$. Let $C_{3}$ and $K^{\prime}$ be as implicitly contained in the statement of Theorem $2^{\prime}$ (recall that $C_{3}$ is 1-1). Set $C_{3}(t)=U(t) C_{3} U(t)^{-1}$.

Then $C_{3}(t) \bar{B}_{C_{1} C_{2}}(t, s) C_{1}(s)^{-1} \in B(X)$ for $a \leqq s \leqq t \leqq b$, and

$$
\left\|C_{3}(t) \bar{B}_{C_{1} C_{2}}(t, s) C_{1}(s)^{-1}\right\| \leqq K^{\prime} K_{0} .
$$

Also, $C_{3}(t) \bar{B}_{C_{1} C_{2}}(t, \cdot) C_{1}(\cdot)^{-1} P(\cdot) x$ is strongly differentiable a.e. on $[a, t]$ with derivative

$$
C_{3} \bar{B}_{C_{1} C_{2}}(t, \cdot) C_{1}(\cdot)^{-1}\left(-\bar{A}(\cdot) P(\cdot)+P^{\prime}(\cdot)\right) x \quad \forall x \in X .
$$

Proof. Since $C_{3}(t) \bar{B}_{C_{1} C_{2}}(t, s) C_{1}(s)^{-1}=U(t)\left(C_{3} B_{C_{1} C_{2}}(t, s) C_{1}^{-1}\right) U(s)^{-1}$, it is bounded by $K^{\prime} K_{0}$ by Theorem 3. Also,

$$
C_{3}(t) \bar{B}_{C_{1} C_{2}}(t, \cdot) C_{1}(\cdot)^{-1} P(\cdot)=U(t)\left(C_{3} B_{C_{1} C_{2}}(t, \cdot) C_{1}^{-1} A\right) U(\cdot)^{-1},
$$

which is absolutely continuous on $[a, t]$ by Theorem 3 , and which is almost everywhere strongly differentiable $\forall x \in X$ with derivative

$$
\begin{aligned}
& U(t)\left(C_{3} B_{C_{1} C_{2}}(t, \cdot) C_{1}^{-1} A\right)^{\prime} U(\cdot)^{-1}+U(t)\left(C_{3} B_{C_{1} C_{2}}(t, \cdot) C_{1}^{-1} A\right) U(\cdot)^{-1 \prime} \\
& \quad=U(t)\left(C_{3} B_{C_{1} C_{2}}(t, \cdot) C_{1}^{-1}\right)\left(\left(-A(\cdot) A U(\cdot)^{-1}-A U(\cdot)^{-1} U^{\prime}(\cdot) U(\cdot)^{-1}\right)\right) \\
& =C_{3}(t) \bar{B}_{C_{1} C_{2}}(t, \cdot) C_{1}(\cdot)^{-1}\left(\left(-U(\cdot) A(\cdot) U(\cdot)^{-1} P(\cdot)-P(\cdot) U^{\prime}(\cdot) U(\cdot)^{-1}\right)\right) .
\end{aligned}
$$

But $A(\cdot)=-U(\cdot)^{-1} U^{\prime}(\cdot)+U(\cdot)^{-1} \bar{A}(\cdot) U(\cdot)$, so that $-U(\cdot) A(\cdot) U(\cdot)^{-1}=$ $U^{\prime}(\cdot) U(\cdot)^{-1}-\bar{A}(\cdot)$, and the expression in double parentheses is $-\bar{A}(\cdot) P(\cdot)+$ $\left(U^{\prime}(\cdot) U(\cdot)^{-1} P(\cdot)-P(\cdot) U^{\prime}(\cdot) U(\cdot)^{-1}\right)$. But since $P(\cdot)=U(\cdot) A U(\cdot)^{-1}$,

$$
\begin{aligned}
P^{\prime}(\cdot) & =U^{\prime}(\cdot) A U(\cdot)^{-1}-U(\cdot) A U(\cdot)^{-1} U^{\prime}(\cdot) U(\cdot)^{-1} \\
& =U^{\prime}(\cdot) U(\cdot)^{-1} P(\cdot)-P(\cdot) U^{\prime}(\cdot) U(\cdot)^{-1}
\end{aligned}
$$

(d) We shall immediately take up some specific cases of the above theorems. We deal first with Kato's basic theorem. Though we do not prove precisely the same result as he does, the change seems in the direction of improvement and generalization.

Consider first a function $N(\cdot) \in L^{\infty}(B(X),[a, b])$ and an associated operator $A \in L(X)$. We assume each $N(t)$ to be in $L(X)$ and to have the same range as $A$. Furthermore, we assume that $\exists M \geqq 1$ such that $\left\|A^{-1} N(\cdot)\right\|_{\infty} \leqq M$ and $\left\|N(\cdot)^{-1} A\right\|_{\infty}$ $\leqq M$ (each $N(t)^{-1} A$ and $A^{-1} N(t)$ is clearly bounded). Finally, we assume that $N(\cdot)$ is of bounded variation productwise over every subinterval of $[a, b]$, with 
variation bounded by $M$. I.e., we assume that for any subdivision $a \leqq t_{0} \leqq t_{1} \leqq \cdots$ $\leqq t_{n} \leqq b, \prod_{i=1}^{n}\left\|N\left(t_{i}\right)^{-1} N\left(t_{i-1}\right)\right\| \leqq M$.

[Digression: Kato assumes that $N(\cdot)^{-1} A$ is of ordinary bounded variation. This implies $N(\cdot)$ is of bounded variation productwise. For (essentially reproducing his proof), if we assume the variation of $N(\cdot)^{-1} A$ to be bounded by $M_{0}$, we have

$$
\begin{aligned}
\left\|N\left(t_{i}\right)^{-1} N\left(t_{i-1}\right)\right\| & =\left\|\left(N\left(t_{i}\right)^{-1}-N\left(t_{i-1}\right)^{-1}\right) N\left(t_{i-1}\right)+1\right\| \\
& \leqq 1+\left\|\left(N\left(t_{i}\right)^{-1}-N\left(t_{i-1}\right)^{-1}\right) A\left(A^{-1} N\left(t_{i-1}\right)\right)\right\| \\
& \leqq 1+\left\|\left(N\left(t_{i}\right)^{-1}-N\left(t_{i-1}\right)^{-1}\right) A\right\| \cdot M
\end{aligned}
$$

so that

$$
\begin{aligned}
\prod_{i=1}^{n}\left\|N\left(t_{i}\right)^{-1} N\left(t_{i-1}\right)\right\| & \leqq \prod_{i=1}^{n}\left(1+M\left\|\left(N\left(t_{i}\right)^{-1}-N\left(t_{i-1}\right)^{-1}\right) A\right\|\right) \\
& \leqq \exp \left(M \sum_{i=1}^{n}\left\|\left(N\left(t_{i}\right)^{-1}-N\left(t_{i-1}\right)^{-1}\right) A\right\|\right) \leqq e^{M M_{0}}
\end{aligned}
$$

since $\ln (x+1) \leqq x$ for $x \geqq 0$, and so the product $\prod_{i=1}^{n}\left(1+x_{i}\right) \leqq \exp \left(\sum_{i=1}^{n} x_{i}\right)$.

However, suppose $f$ is a function on $[a, b]$ with values in $[0, \pi / 2]$ (where $\sin x \geqq x / 2)$ which is not of bounded variation, and we set $N(t)=e^{i f(t)} A$. Since $N\left(t_{i}\right)^{-1} N\left(t_{i-1}\right)=\exp \left(i\left(f\left(t_{i-1}\right)-f\left(t_{i}\right)\right)\right),\left\|N\left(t_{i}\right)^{-1} N\left(t_{i-1}\right)\right\|=1$, so that $N(\cdot)$ is of bounded variation productwise over every subinterval of $[a, b]$ with bound 1 . But

$$
\begin{aligned}
\left\|\left(N\left(t_{i}\right)^{-1}-N\left(t_{i-1}\right)^{-1}\right) A\right\| & =\left\|e^{-i f\left(t_{i}\right)}-e^{-i f\left(t_{i-1}\right)}\right\|=\left\|1-e^{i\left(f\left(t_{i}\right)-f\left(t_{i-1}\right)\right)}\right\| \\
& =\left(\left(1-\cos \left(f\left(t_{i}\right)-f\left(t_{i-1}\right)\right)\right)^{2}+\sin ^{2}\left(f\left(t_{i}\right)-f\left(t_{i-1}\right)\right)\right)^{1 / 2} \\
& \geqq \sin \left|f\left(t_{i}\right)-f\left(t_{i-1}\right)\right| \geqq \frac{1}{2}\left|f\left(t_{i}\right)-f\left(t_{i-1}\right)\right|,
\end{aligned}
$$

so that $N(\cdot)^{-1} A$ is not of bounded variation.]

Suppose now that $A(\cdot) \in L^{1}(B(X),[a, b])$, and that for each $t, A(t)$ preserves the range of $A$ in such a way that $\tilde{A}(\cdot)=A^{-1} A(\cdot) A$ belongs to $L^{1}(B(X),[a, b])$. Then for the associated $\widetilde{B}(t, s), a \leqq s \leqq t \leqq b, B(t, s)$ preserves the range of $A$ in such a way that $\tilde{B}(t, s)=A^{-1} B(t, s) A$. For if we consider $A(t)$ as an operator $A_{0}(t)$ in the Banach space $R(A), A_{0}(\cdot) \in L^{1}(R(A),[a, b])$, and there exists an associated $B_{0}(t, s)$ for $a \leqq s \leqq t \leqq b$. For $x \in R(A), B_{0}(\cdot, s) x$ is absolutely continuous in the norm of $R(A)$, and, hence, in the norm of $X$. Since it is strongly differentiable a.e. on $[s, b]$ in the norm of $R(A)$ with derivative $A_{0}(\cdot) B_{0}(\cdot, s) x=A(\cdot) B_{0}(\cdot, s) x$, the same holds in the norm of $X$, so that $B_{0}(\cdot, s) x=B(\cdot, s) x$. Thus $B_{0}(t, s)=B(t, s) \mid R(A) . B_{0}(\cdot, s)$ is absolutely continuous on $[s, b]$ in the norm of $R(A)$, i.e., $A^{-1} B_{0}(\cdot, s) A$ $=A^{-1} B(\cdot, s) A$ is absolutely continuous in the norm of $X$ on $[s, b]$. Similarly, $B_{0}(\cdot, s) x$ is a.e. strongly differentiable in the norm of $R(A) \quad \forall x \in R(A)$ with derivative $A_{0}(\cdot) B_{0}(\cdot, s) x=A(\cdot) B(\cdot, s) x$, so that $A^{-1} B(\cdot, s) A x$ is a.e. 'strongly differentiable in the norm of $X \quad \forall x \in X$ with derivative $\left(A^{-1} A(\cdot) A\right)\left(A^{-1} B(\cdot, s) A\right) x$. 
Thus $\widetilde{B}(t, s)=A^{-1} B(t, s) A$ by uniqueness. Let us consider $\left\|A^{-1} B(t, s) A\right\|$ $=\|\widetilde{B}(t, s)\|$. Recall that $\widetilde{B}(\cdot, s)=I+\sum_{k=1}^{\infty} B_{k}(\cdot)$, where $B_{k}(t)$ is defined by

$$
\begin{aligned}
B_{k}(t) x= & \int_{t \geq t_{n} \geq \ldots \geq t_{1} \geq_{s}} \tilde{A}\left(t_{n}\right) \cdots \tilde{A}\left(t_{1}\right) x d t_{1} \cdots d t_{n} \\
= & \int_{t \geq t_{n} \geq \ldots \geq t_{1} \geq_{s}}\left(\left(A^{-1} N\left(t_{n}\right)\right) \cdot\left(N\left(t_{n}\right)^{-1} A\left(t_{n}\right) N\left(t_{n}\right)\right) \cdot\left(N\left(t_{n}\right)^{-1} N\left(t_{n-1}\right)\right)\right. \\
& \cdot\left(N\left(t_{n-1}\right)^{-1} A\left(t_{n-1}\right) N\left(t_{n-1}\right)\right) \cdots\left(N\left(t_{2}\right)^{-1} N\left(t_{1}\right)\right) \\
& \left.\cdot\left(N\left(t_{1}\right)^{-1} A\left(t_{1}\right) N\left(t_{1}\right)\right) \cdot\left(N\left(t_{1}\right)^{-1} A\right) x\right) d t_{1} \cdots d t_{n} .
\end{aligned}
$$

Thus

$$
\begin{aligned}
\left\|\widetilde{B}_{k}(t) x\right\| \leqq & M^{3}\|x\| \int_{s}^{t}\left\|N\left(t_{n}\right)^{-1} A\left(t_{n}\right) N\left(t_{n}\right)\right\| d t_{n} \\
& \cdot \int_{8}^{t_{n}}\left\|N\left(t_{n-1}\right)^{-1} A\left(t_{n-1}\right) N\left(t_{n-1}\right)\right\| d t_{n-1} \cdots \int_{8}^{t_{2}}\left\|N\left(t_{1}\right)^{-1} A\left(t_{1}\right) N\left(t_{1}\right)\right\| d t_{1}
\end{aligned}
$$

by boundedness of product variation of $N(\cdot)$ by $M$ and by $\left\|A^{-1} N(\cdot)\right\|_{\infty}$ and $\left\|N(\cdot)^{-1} A\right\|_{\infty} \leqq M$. Thus exactly as in the first lemma for the Cauchy problem for bounded operators,

$$
\left\|\tilde{B}_{k}(t)\right\| \leqq\left(M^{3} / n !\right)\left(\int_{s}^{t}\left\|N(r)^{-1} A(r) N(r)\right\| d r\right)^{n},
$$

so that

$$
\|\tilde{B}(t, s)\| \leqq M^{3} \exp \left(\int_{s}^{t}\left\|N(r)^{-1} A(r) N(r)\right\| d r\right)
$$

since $M \geqq 1$. Exactly as in the boundedness proposition we get

$$
\left\|e^{n(t-s)} \tilde{B}(t, s)\right\| \leqq M^{3} \exp \left(\int_{s}^{t}\left\|N(r)^{-1}(A(r)+n) N(r)\right\| d r\right),
$$

so that

$$
\|\tilde{B}(t, s)\| \leqq M^{3} \exp \left(\int_{s}^{t}\left(\left\|N(r)^{-1} A(r) N(r)+n\right\|-n\right) d r\right),
$$

and

$$
\|\widetilde{B}(t, s)\| \leqq M^{3} \exp \left(\int_{s}^{t} \rho\left(N(r)^{-1} A(r) N(r)\right) d r\right) .
$$

We may now prove the following theorem.

THEOREM. Let $A \in L(X)$. Let $U(\cdot) \in L^{\infty}(B(X),[a, b])$ be absolutely continuous with each $U(t)$ invertible, and let $U(\cdot) x$ be a.e. strongly differentiable $\forall x \in X$ with derivative $U^{\prime}(\cdot) x$, where $U^{\prime}(\cdot) \in L^{1}(B(X),[a, b])$. If $P(t)=U(t) A U(t)^{-1}$, assume that $U^{\prime}(t)$ takes the range of $A$ into the domain of $P(t)^{-1}$ in such a way that

$$
P(\cdot)^{-1} U^{\prime}(\cdot) A \in L^{1}(B(X),[a, b]) \text {. }
$$


Suppose now that $\bar{A}(\cdot)$ is a function from $[a, b]$ to $C D(X)$ with the following properties:

(1) the range of $P(t)$ is the domain of $\bar{A}(t)$, and $\bar{A}(\cdot) P(\cdot)$ belongs to $L^{1}(B(X),[a, b])$,

(2) the spectrum of $\bar{A}(t)$ lies in a left half-plane,

(3) $\exists m(\cdot) \in L^{1}([a, b])$ and strictly positive $\varepsilon(\cdot)$ with $\varepsilon(\cdot)^{-1} \in L^{1}([a, b])$ such that for almost all $t, 0 \leqq \varepsilon \leqq \varepsilon(t)$ entails $\left\|U(t)^{-1}(1-\varepsilon \bar{A}(t))^{-1} U(t)\right\| \leqq 1+m(t) \varepsilon$,

(4) there exists a measurable function $n(\cdot)$ on $[a, b]$ such that

$$
N(\cdot)=U(\cdot)^{-1}(1-n(\cdot) \bar{A}(\cdot))^{-1} U(\cdot)
$$

(which has the same range as $A$ ) has $N(\cdot)^{-1} A$ and $A^{-1} N(\cdot)$ belonging to $L^{\infty}(B(X),[a, b])$, and has product variation over every subinterval of $[a, b]$ bounded by a constant $M$.

Then for $a \leqq s \leqq t \leqq b \quad \exists \bar{B}(t, s) \in B(X)$ simultaneously strongly continuous in $s$ and $t$ with $\|\bar{B}(t, s)\| \leqq\|U(t)\| \exp \left(\int_{s}^{t}\left(m(r)+\left\|U(r)^{-1} U^{\prime}(r)\right\| d r\right)\right)$ such that

(1) $\bar{B}(t, t)=I \quad \forall t \in[a, b]$,

(2) $\bar{B}(t, s) P(s)$ has range in the domain of $P(t)^{-1}$, and $P(\cdot)^{-1} \bar{B}(\cdot, s) P(s)$ $\in L^{\infty}(B(X),[s, b])$,

(3) $\bar{B}(\cdot, s) P(s)$ is absolutely continuous on $[s, b]$, and $\bar{B}(\cdot, s) P(s) x$ is strongly differentiable a.e. on $[s, b] \quad \forall x \in X$ with derivative $\bar{A}(\cdot) \bar{B}(\cdot, s) P(s) x$,

(4) $\bar{B}(t, \cdot) P(\cdot)$ is absolutely continuous on $[a, t]$, and $\bar{B}(t, \cdot) P(\cdot) x$ is differentiable a.e. $\forall x \in X$ with derivative $\bar{B}(t, \cdot)\left(P^{\prime}(\cdot)-\bar{A}(\cdot) P(\cdot)\right) x$,

(5) $\bar{B}\left(t, s_{0}\right) \bar{B}\left(s_{0}, s\right)=\bar{B}(t, s)$ for $a \leqq s \leqq s_{0} \leqq t \leqq b$.

$\bar{B}(\cdot, s) \in L^{\infty}(B(X),[s, b])$ is uniquely characterized by properties (1), (2), and (3).

Proof. Set $A(\cdot)=-U(\cdot)^{-1} U^{\prime}(\cdot)+U(\cdot)^{-1} \bar{A}(\cdot) U(\cdot)$. We shall show that $A(\cdot)$ satisfies the hypotheses of Theorem 1 with $C_{1}=I$ and $C_{2}=A$.

Let $\varepsilon_{0}(t)=\min \left\{\varepsilon(t),|2 m(t)|^{-1}\right\}$, and set $\varepsilon_{n}(t)=\varepsilon_{0}(t) / n$. Set $A_{n}(t)=-U(t)^{-1} U^{\prime}(t)$ $+U(t)^{-1} \bar{A}(t)\left(1-\varepsilon_{n}(t) \bar{A}(t)\right)^{-1} U(t)$. We have

so that

$$
\begin{aligned}
\left\|A_{n}(t) A\right\| \leqq & \left\|U(t)^{-1}\right\| \cdot\left\|U^{\prime}(t)\right\| \cdot\|A\| \\
& +\left\|U(t)^{-1}\left(1-\varepsilon_{n}(t) \bar{A}(t)\right)^{-1} U(t)\right\| \cdot\left\|U(t)^{-1}\right\| \cdot\|\bar{A}(t) P(t)\| \cdot\|U(t)\|,
\end{aligned}
$$

$$
\left\|A_{n}(t) A\right\| \leqq\left\|U(\cdot)^{-1}\right\|_{\infty}\|A\|\left\|U^{\prime}(t)\right\|+(1+1 / 2 n)\left\|U(\cdot)^{-1}\right\|_{\infty}\|U(\cdot)\|_{\infty}\|\bar{A}(t) P(t)\|,
$$

and, similarly,

$$
\|A(\cdot) A\| \leqq\left\|U(\cdot)^{-1}\right\|_{\infty}\|A\|\left\|U^{\prime}(t)\right\|+\left\|U(\cdot)^{-1}\right\|_{\infty}\|U(\cdot)\|_{\infty}\|\bar{A}(t) P(t)\| .
$$

Thus $\exists k(\cdot) \in L^{1}([a, b])$ with $\left\|A_{n}(\cdot) A\right\|+\|A(\cdot) A\| \leqq k(\cdot)$. Since

we have

$$
\bar{A}(t)\left(1-\varepsilon_{n}(t) \bar{A}(t)\right)^{-1}=\varepsilon_{n}(t)^{-1}\left(\left(1-\varepsilon_{n}(t) \bar{A}(t)\right)^{-1}-I\right),
$$

$$
\begin{aligned}
\left\|A_{n}(t)\right\| & \leqq\left\|U(\cdot)^{-1}\right\|_{\infty}\left\|U^{\prime}(t)\right\|+8 \varepsilon_{n}(t)^{-1} \\
& \leqq\left\|U(\cdot)^{-1}\right\|_{\infty}\left\|U^{\prime}(t)\right\|+8 n\left(2 m(t)+e(t)^{-1}\right),
\end{aligned}
$$


and each $A_{n}(\cdot) \in L^{1}(B(X),[a, b])$. We may take $A_{n}(\cdot)$ to be as in Theorem 1 , then, and we have already verified hypothesis (3) of that theorem.

Let us verify hypothesis (1) of Theorem 1 , that the range of $B_{n}(t, s) A$ is in the domain of $A^{-1} \forall n$ and $a \leqq s \leqq t \leqq b$. By the remarks preceding this theorem it suffices to show that $A_{n}(t)$ preserves the range of $A$ in such a way that $A^{-1} A_{n}(\cdot) A \in$ $L^{1}(B(X),[a, b])$. Since $P(\cdot)^{-1}=U(\cdot) A^{-1} U(\cdot)^{-1}, P(\cdot)^{-1} U^{\prime}(\cdot) A \in L^{1}(B(X),[a, b])$, so that $U(\cdot) A^{-1} U(\cdot)^{-1} U^{\prime}(\cdot) A \in L^{1}(B(X),[a, b]),-A^{-1} U(\cdot)^{-1} U^{\prime}(\cdot) A \in$ $L^{1}(B(X),[a, b])$. For the other term,

$$
\begin{aligned}
&\left\|A^{-1} U(t)^{-1}\left(1-\varepsilon_{n}(t) \bar{A}(t)\right)^{-1} \bar{A}(t) U(t) A\right\| \\
& \leqq\left\|A^{-1} N(t)\right\| \cdot\left\|U(t)^{-1}\left(1-\varepsilon_{n}(t) \bar{A}(t)\right)^{-1} \bar{A}(t) U(t)\right\| \cdot\left\|N(t)^{-1} A\right\| \\
& \leqq\left\|A^{-1} N(\cdot)\right\|_{\infty}\left\|N(\cdot)^{-1} A\right\|_{\infty} B n\left(2 m(t)+\varepsilon(t)^{-1}\right) .
\end{aligned}
$$

Let us now verify hypothesis (2) of Theorem 1 for $A(\cdot)$. We have, first, for uniform boundedness of $B_{n}(t, s)$, only to show that all of the $\rho\left(A_{n}(\cdot)\right)$ are bounded from above by a fixed function in $L^{1}([a, b])$. We have

$$
\begin{aligned}
\rho\left(A_{n}(t)\right) \leqq & \rho\left(U(t)^{-1} U^{\prime}(t)\right)+\rho\left(U(t)^{-1} \bar{A}(t)\left(1-\varepsilon_{n}(t) \bar{A}(t)\right)^{-1} U(t)\right) \\
\leqq & \left\|U(t)^{-1}\right\|\left\|U^{\prime}(t)\right\| \\
& +\left(\left\|U(t)^{-1} \bar{A}(t)\left(1-\varepsilon_{n}(t) \bar{A}(t)\right)^{-1} U(t)+\varepsilon_{n}(t)^{-1}\right\|-\varepsilon_{n}(t)^{-1}\right) \\
= & \left\|U(t)^{-1}\right\|\left\|U^{\prime}(t)\right\|+\left(\varepsilon_{n}(t)^{-1}\left\|U(t)^{-1}\left(1-\varepsilon_{n}(t) \bar{A}(t)\right)^{-1} U(t)\right\|-\varepsilon_{n}(t)^{-1}\right) \\
\leqq & \left\|U(t)^{-1}\right\|\left\|U^{\prime}(t)\right\|+\varepsilon_{n}(t)^{-1}\left(1+\varepsilon_{n}(t) m(t)\right)-\varepsilon_{n}(t)^{-1} \\
= & \left\|U(t)^{-1}\right\|\left\|U^{\prime}(t)\right\|+m(t)
\end{aligned}
$$

so that

$$
\left\|B_{n}(t, s)\right\| \leqq \exp \left(\int_{s}^{t}\left(m(r)+\left\|U(r)^{-1}\right\|\left\|U^{\prime}(r)\right\|\right) d r\right) .
$$

To show uniform boundedness of $A^{-1} B_{n}(t, s) A$, we have, by the remarks directly preceding the theorem, only to show that $\rho\left(N(t)^{-1} A_{n}(t) N(t)\right)$ is bounded from above by a fixed function in $L^{1}([a, b])$. But

$$
\begin{aligned}
\rho\left(N(t)^{-1} A_{n}(t) N(t)\right) \leqq & \rho\left(N(t)^{-1} U(t)^{-1} U^{\prime}(t) N(t)\right) \\
& +\rho\left(N(t)^{-1} U(t)^{-1} \bar{A}(t)\left(1-\varepsilon_{n}(t) \bar{A}(t)\right)^{-1} U(t) N(t)\right) .
\end{aligned}
$$

Since $N(t)=U(t)^{-1}(1-n(t) \bar{A}(t))^{-1} U(t), N(t)$ commutes through the argument of the last term, so that

$$
\begin{aligned}
\rho\left(N(t)^{-1}\right. & \left.A_{n}(t) N(t)\right) \\
\leqq & \left\|N(t)^{-1} A\right\|\left\|A^{-1} U(t)^{-1} U^{\prime}(t) A\right\|\left\|A^{-1} N(t)\right\| \\
& +\rho\left(U(t)^{-1} \bar{A}(t)\left(1-\varepsilon_{n}(t) \bar{A}(t)\right)^{-1} U(t)\right) \\
\leqq & \left\|N(\cdot)^{-1} A\right\|_{\infty}\left\|U(\cdot)^{-1}\right\|_{\infty}\left\|P(t)^{-1} U^{\prime}(t) A\right\|\left\|A^{-1} N(\cdot)\right\|_{\infty}+m(t),
\end{aligned}
$$

as before. 
Let us now verify hypothesis (4) of Theorem 1 , that $\left(A_{n}(t) A\right)^{*} \rightarrow(A(t) A)^{*}$ strongly for almost all $t \in[a, b]$. First, $\left\|\left(A_{n}(t) A\right)^{*}\right\|=\left\|A_{n}(t) A\right\|$ is uniformly bounded in $\boldsymbol{n}$ for fixed $t$. For

$$
\begin{aligned}
\left\|A_{n}(t) A\right\| & \leqq\left\|U(t)^{-1} U^{\prime}(t) A\right\|+\left\|U(t)^{-1}\left(1-\varepsilon_{n}(t) \bar{A}_{n}(t)\right)^{-1} \bar{A}(t) U(t) A\right\| \\
& \leqq\left\|U(t)^{-1} U^{\prime}(t) A\right\|+\left(1+\varepsilon_{n}(t) m(t)\right)\left\|U(t)^{-1}\right\|\|\bar{A}(t) P(t)\|\|U(t)\|,
\end{aligned}
$$

and $\left(1+\varepsilon_{n}(t) m(t)\right) \leqq 1+1 / 2 n<2$. Thus it suffices to show strong convergence on a dense subspace of $X^{*}$, for example, the set of $f \circ N(t)$. Clearly, it suffices to show that

$$
(f \circ N(t)) \circ\left(U(t)^{-1} \bar{A}(t)\left(1-\varepsilon_{n}(t) \bar{A}(t)\right)^{-1} U(t)\right) A \rightarrow(f \circ N(t)) \circ\left(U(t)^{-1} \bar{A}(t) U(t)\right) A .
$$

I.e., we must show that $f \circ\left(N(t) U(t)^{-1} \bar{A}(t)\right) \circ \varepsilon_{n}(t) \bar{A}(t)\left(1-\varepsilon_{n}(t) \bar{A}(t)\right)^{-1} \circ U(t) A$ goes to zero. Since $\varepsilon_{n}(t) \rightarrow 0$, it suffices to show that

$$
f \circ\left(N(t) U(t)^{-1} \bar{A}(t)\right) \circ \bar{A}(t)\left(1-\varepsilon_{n}(t) \bar{A}(t)\right)^{-1} \circ U(t) A
$$

is uniformly bounded. But

$$
N(t) U(t)^{-1} \bar{A}(t)=U(t)^{-1}(1-n(t) \bar{A}(t))^{-1} \bar{A}(t)=U(t)^{-1} \bar{A}(t)(1-n(t) \bar{A}(t))^{-1},
$$

so that this is bounded, $\bar{A}(t) U(t) A=\bar{A}(t) P(t) U(t)$, so that this is bounded, and

$$
\begin{aligned}
\left\|\left(1-\varepsilon_{n}(t) \bar{A}(t)\right)^{-1}\right\| & \leqq\|U(t)\|\left\|U(t)^{-1}\right\|(1+1 / 2 n) \\
& <2\|U(t)\|\left\|U(t)^{-1}\right\| \quad \forall n .
\end{aligned}
$$

Thus by Theorem 1', (1), (2), and (3) of this theorem hold. To verify the hypotheses of Theorem 2', and, a fortiori, of Theorem $3^{\prime}$, we need only show that $A_{n}(t) A \rightarrow A(t) A$ strongly for fixed $t$. Since we have already shown that $\left\|A_{n}(t) A\right\|$ is uniformly bounded in $n$, it suffices to show strong convergence on elements of the form $A^{-1} N(t) A x$, i.e., it suffices to show that $A_{n}(t) N(t) A x$ converges strongly to $A(t) N(t) A x \quad \forall x \in X$. As before,

$$
\begin{aligned}
\left(A_{n}(t) N(t)-A(t) N(t)\right) A x \\
=\left(U(t)^{-1}\right)(\bar{A}(t) U(t) N(t)) U(t)^{-1} \varepsilon_{n}(t)\left(1-\varepsilon_{n}(t) \bar{A}(t)\right)^{-1} \bar{A}(t) U(t) A x \\
=\varepsilon_{n}(t)\left(U(t)^{-1}(\bar{A}(t) U(t) A)\left(A^{-1} N(t)\right) U(t)^{-1}\left(1-\varepsilon_{n}(t) \bar{A}(t)\right)^{-1}(\bar{A}(t) U(t) A) x\right),
\end{aligned}
$$

and $\varepsilon_{n}(t) \rightarrow 0$, while the other term remains bounded by

$$
\left\|U(t)^{-1}\right\|^{2}\|\bar{A}(t) P(t)\|\|U(t)\|\left\|A^{-1} N(t)\right\| 2\|U(t)\|\left\|U(t)^{-1}\right\|\|\bar{A}(t) P(t)\|\|U(t)\|\|x\| .
$$

Thus Theorems $2^{\prime}$ and $3^{\prime}$ and Corollary $2^{\prime}$ hold, proving (4), (5), and the uniqueness assertion of this theorem. The bounds mentioned have been proved in passing. Only joint strong continuity of $\bar{B}(t, s)$ in $s$ and $t$ remains to be shown. Assuming for convenience that $\delta$ and $\delta^{\prime}$ are small and $\geqq 0$, we have for $x \in X$,

$$
\begin{aligned}
\left\|\left(\bar{B}\left(t+\delta, s+\delta^{\prime}\right)-\bar{B}(t, s)\right) x\right\| \leqq & \|(\bar{B}(t+\delta, s)-\bar{B}(t, s)) x\| \\
& +\left\|\bar{B}\left(t+\delta, s+\delta^{\prime}\right)\right\|\left\|\left(\bar{B}\left(s+\delta^{\prime}, s\right)-I\right) x\right\| .
\end{aligned}
$$


(e) We shall now take up some specific cases of the existence theorems in the case that $X$ is a Hilbert space. In this case approximation of unbounded operators by use of projections on which they are bounded seems most natural and seems, at least, to be possible in most of the cases where approximations using resolvents can be used.

Because the additional hypothesis that $X$ is a Hilbert space makes possible a number of existence theorems, we shall treat only two relatively simple ones by way of example.

THEOREM. Let $A \in L(X)$ be self-adjoint. Let $U(\cdot) \in L^{\infty}(B(X),[a, b])$ have each $U(t)$ unitary, and let $U(\cdot) x$ be a.e. strongly differentiable $\forall x \in X$ with derivative $U^{\prime}(\cdot) x$, where $U^{\prime}(\cdot) \in L^{1}(B(X),[a, b])$. If $P(\cdot)=U(\cdot) A U(\cdot)^{-1}$, assume that $U^{\prime}(t)$ takes the range of $A$ into the domain of $P(\cdot)$ in such fashion that $P(\cdot)^{-1} U^{\prime}(\cdot) A$ $\in L^{1}(B(X),[a, b])$.

Suppose now that $\bar{A}(\cdot)$ is a function from $[a, b]$ to $C D(X)$ with the following properties:

(1) each $\bar{A}(t)$ is skew-adjoint,

(2) the domain of $\bar{A}(t)$ contains the range of $P(t)$, and $\bar{A}(\cdot) P(\cdot) \in L^{1}(B(X),[a, b])$,

(3) $\exists m(\cdot) \in L^{1}([a, b])$ such that $\left[\bar{A}(\cdot), P(\cdot)^{2}\right] \leqq m(\cdot) P(\cdot)^{2}$.

Then for $a \leqq s \leqq t \leqq b \quad \exists \bar{B}(t, s) \in B(X)$ jointly strongly continuous in $t$ and $s$ such that $\bar{B}(t, s)$ is isometric, and

(1) $\bar{B}(t, t)=I \quad \forall t \in[a, b]$,

(2) $\bar{B}(t, s)$ takes the range of $P(s)$ into the domain of $P(t)^{-1}$, and

$$
P(\cdot)^{-1} \bar{B}(\cdot, s) P(s) \in L^{\infty}(B(X),[s, b]),
$$

(3) $\bar{B}(\cdot, s) P(s)$ is absolutely continuous on $[s, b]$, and $\bar{B}(\cdot, s) P(s) x$ is strongly differentiable a.e. on $[s, b] \quad \forall x \in X$ with derivative $\bar{A}(\cdot) \bar{B}(\cdot, s) P(s) x$,

(4) $\bar{B}(t, \cdot) P(\cdot)$ is absolutely continuous on $[a, t]$, and $\bar{B}(t, \cdot) P(\cdot) x$ is strongly differentiable a.e. $\forall x \in X$ with derivative $\bar{B}(t, \cdot)\left(P^{\prime}(\cdot)-\bar{A}(\cdot) P(\cdot)\right) x$,

(5) $\bar{B}\left(t, s_{0}\right) \bar{B}\left(s_{0}, s\right)=\bar{B}(t, s)$ for $a \leqq s \leqq s_{0} \leqq t \leqq b$.

$\bar{B}(\cdot, s) \in L^{\infty}(B(X),[s, b])$ is uniquely characterized by properties (1), (2), and (3).

Proof. Note first that since $U(t) x=U(s) x+\int_{s}^{t} U^{\prime}(r) x d r \quad \forall x \in X$ entails $U(t)^{*} x=U(s)^{*} x+\int_{s}^{t}\left(U^{\prime}(r)\right)^{*} x d r \quad \forall x \in X$ by the discussion for bounded operators in Hilbert space, $U^{\prime}(\cdot)^{*}$ is the strong derivative of $U(\cdot)^{-1}$, so that $U^{\prime}(\cdot)^{*}$ $=-U(\cdot)^{-1} U^{\prime}(\cdot) U(\cdot)^{-1}$. Thus $\left(U(t)^{-1} U^{\prime}(t)\right)^{*}=U^{\prime}(t)^{*} U(t)=-U(t)^{-1} U^{\prime}(t)$, so that each $U(t)^{-1} U^{\prime}(t)$ is skew-adjoint.

Let us show that the hypotheses of Theorem 1 hold for $A(\cdot)=-U(\cdot)^{-1} U^{\prime}(\cdot)$ $+U(\cdot)^{-1} \bar{A}(\cdot) U(\cdot)$ with $C_{1}=I$ and $C_{2}=A$.

Since

$$
\begin{aligned}
\|A(t) A\| & \leqq\left\|U(t)^{-1} U^{\prime}(t) A\right\|+\left\|U(t)^{-1} \bar{A}(t) P(t) U(t)\right\| \\
& \leqq\left\|U^{\prime}(t)\right\|\|A\|+\|\bar{A}(t) P(t)\|,
\end{aligned}
$$

we have $A(\cdot) A \in L^{1}(B(X),[a, b])$. 
If $\left\{E_{\lambda}\right\}$ is the spectral family for $A$, set $P_{n}=\left(1-E_{1 / n}\right)+E_{-1 / n}$. Then $A^{-1} P_{n} \in B(X)$, and so $\left\|A(t) P_{n}\right\| \leqq\left\|A(t) A\left(A^{-1} P_{n}\right)\right\| \leqq n\|A(t) A\|$. Set $A_{n}(\cdot)=P_{n} A(\cdot) P_{n}$. Then each $A_{n}(t)$ is skew-adjoint because each $A(\cdot)$ is, and $A_{n}(\cdot) \in L^{1}(B(X),[a, b]) \quad \forall n$. Since, by the discussion for bounded operators in Hilbert space, each $B_{n}(t, s)$ is thus unitary, $\left\|B_{n}(t, s)\right\| \leqq 1 \quad \forall n, a \leqq s \leqq t \leqq b$. Thus the first part of hypothesis (2) of Theorem 1 is satisfied.

Since the range of $A_{n}(t)$ lies in the range of $P_{n}$, which lies in the domain of $A^{-1}$, we may consider $A^{-1} A_{n}(\cdot) A$. By an earlier discussion, $A^{-1} A_{n}(\cdot) A \in L^{1}(B(X),[a, b])$ will imply that the range of $B_{n}(t, s) A$ lies in the domain of $A^{-1}$, thus proving hypothesis (1) of Theorem 1. But $\left\|\left(A^{-1} P_{n}\right) A(\cdot)\left(P_{n} A\right)\right\| \leqq\left\|A^{-1} P_{n}\right\|\|A(\cdot) A\|\left\|P_{n}\right\|$ $\leqq n\|A(\cdot) A\|$.

Let us now verify the remainder of hypothesis (2) of Theorem 1 , that $A^{-1} B_{n}(t, s) A$ is uniformly bounded. We compute $\rho\left(A^{-1} A_{n}(\cdot) A\right)$. We have

$$
\begin{aligned}
& \rho\left(A^{-1} A_{n}(t) A\right)=\rho\left(A^{-1} P_{n} A(t) A P_{n}\right) \\
& \leqq \rho\left(-P_{n} A^{-1} U(t)^{-1} U^{\prime}(t) A P_{n}\right)+\rho\left(\left(A^{-1} P_{n}\right) U(t)^{-1} \bar{A}(t) U(t)\left(A P_{n}\right)\right) \\
& \leqq\left\|P(t)^{-1} U^{\prime}(t) A\right\| \\
&+\frac{1}{2} \sup \sigma\left(\left(A^{-1} P_{n}\right) U(t)^{-1} \bar{A}(t) U(t)\left(A P_{n}\right)-\left(A P_{n}\right) U(t)^{-1} \bar{A}(t) U(t)\left(A^{-1} P_{n}\right)\right) .
\end{aligned}
$$

Since

$$
\bar{A}(t) P(t)^{2}-P(t)^{2} \bar{A}(t) \leqq m(t) P(t)^{2},
$$

or

$$
\bar{A}(t) U(t) A^{2} U(t)^{-1}-U(t) A^{2} U(t)^{-1} \bar{A}(t) \leqq m(t) U(t) A^{2} U(t)^{-1},
$$

or

$$
U(t)^{-1} \bar{A}(t) U(t) A^{2}-A^{2} U(t)^{-1} \bar{A}(t) U(t) \leqq m(t) A^{2},
$$

it follows that

$$
P_{n} U(t)^{-1} \bar{A}(t) U(t) A^{2} P_{n}-P_{n} A^{2} U(t)^{-1} \bar{A}(t) U(t) P_{n} \leqq m(t) A^{2} P_{n},
$$

and, therefore,

$$
\left(A^{-1} P_{n}\right) U(t)^{-1} \bar{A}(t) U(t)\left(A P_{n}\right)-\left(A P_{n}\right) U(t)^{-1} \bar{A}(t) U(t)\left(A^{-1} P_{n}\right) \leqq m(t) P_{n} .
$$

Thus $\rho\left(A^{-1} A_{n}(\cdot) A\right) \leqq\left\|P(\cdot)^{-1} U^{\prime}(\cdot) A\right\|+\frac{1}{2} m(\cdot)$, and

$$
\begin{aligned}
\left\|A^{-1} B_{n}(t, s) A\right\| & \leqq \exp \left(\int_{s}^{t}\left(\frac{1}{2} m(r)+\left\|P(r)^{-1} U^{\prime}(r) A\right\|\right) d r\right) \\
& \leqq \exp \left(\frac{1}{2}\|m(\cdot)\|_{1}+\left\|P(\cdot)^{-1} U^{\prime}(\cdot) A\right\|_{1}\right) .
\end{aligned}
$$

For hypothesis (3) of Theorem 1, we have $\left\|A_{n}(t) A\right\|=\left\|P_{n} A(t) A P_{n}\right\| \leqq\|A(t) A\|$, so that $k(\cdot)=2\|A(\cdot) A\| \in L^{1}([a, b])$ satisfies $\left\|A_{n}(\cdot) A\right\|+\|A(\cdot) A\| \leqq k(\cdot)$.

For hypothesis (4) of Theorem 1 we must show that $\left(A_{n}(t) A\right)^{*}$ converges strongly 
to $(A(t) A)^{*}$ for almost all $t$. But $\left\|\left(A_{n}(t) A\right)^{*}\right\|=\left\|A_{n}(t) A\right\| \leqq\|A(t) A\|$, so that $\left\|\left(A_{n}(t) A\right)^{*}\right\|$ is uniformly bounded for fixed $t$. Thus we need only show that $A A_{n}(t)$ $\rightarrow \overline{A A(t)}$ on a dense subset of $X$. For $x$ in the range of $P_{k}$ and for $n \geqq k, A A_{n}(t) x$ $=\left(A P_{n}\right) A(t) x$, and $A P_{n} \rightarrow A$ strongly.

Thus conclusions (1), (2), and (3) of this theorem together with strong continuity of $\bar{B}(\cdot, s)$ follow from Theorem $1^{\prime}$. To verify the hypotheses of Theorem 2 , and, a fortiori, of Theorem 3 , for $A(\cdot)$, we need only show that $A_{n}(t) A \rightarrow A(t) A$ strongly for almost all $t$. Again by uniform boundedness of $\left\|A_{n}(t) A\right\|$ for fixed $t$, it suffices to show strong convergence on $x$ in the range of $P_{k}$ for some $k$. For $n \geqq k, A_{n}(t) A x$ $\rightarrow P_{n} A(t) A x$, and $P_{n} \rightarrow I$ strongly.

Thus the uniqueness assertion holds by Corollary $1^{\prime}$, conclusion (4) holds by Theorem 3', and conclusion (5) holds by Corollary 2'. As before, joint strong continuity of $\bar{B}(t, s)$ in $t$ and $s$ follows from conclusion (5), boundedness of $\bar{B}(t, s)$, and strong continuity in $t$.

It remains only to show that $\bar{B}(t, s)$ is isometric. But

$$
\begin{aligned}
(\bar{B}(\cdot, s) P(s) x, \bar{B}(\cdot, s) P(s) x)^{\prime} \\
\quad=(\bar{A}(\cdot) \bar{B}(\cdot, s) P(s) x, \bar{B}(\cdot, s) P(s) x)+(\bar{B}(\cdot, s) P(s) x, \bar{A}(\cdot) \bar{B}(\cdot, s) P(s) x)=0
\end{aligned}
$$

$\forall x \in X$, so that $\|\bar{B}(t, s) P(s) x\|^{2}=\|P(s) x\|^{2} \quad \forall x \in X$.

None of the examples up to now has made use of the auxiliary operator $C_{1}$ of Theorem 1. In the following example it is used to control the bounds of the $B_{n}(t, s)$ which appear. This time we do not attempt a formulation in terms of the generalized theorems.

THEOREM. Let $\mathscr{A}$ be a weakly-closed ring of operators in $X$ with commutor $\mathscr{A}$. Let $A(\cdot)$ be a function from $[a, b]$ to $C D(X)$ such that each $A(t)$ is affiliated with $\mathscr{A}$. Suppose $\exists A \in \tilde{A} \cap L(X)$ (which by polar decomposition we may take to be positive self-adjoint) such that the range of $A$ lies in the domain of each $A(t)$, and $A(\cdot) A$ $\in L^{1}(B(X),[a, b])$.

Then $\exists C \in \mathscr{A}^{+} \cap L(X)$ such that $C=g(A)$ for some nonnegative Borel function $g$, and for $a \leqq s \leqq t \leqq b \quad \exists B(t, s)$ in the weakly-closed ring $\mathscr{A}^{\prime}$ generated by $\mathscr{A}$ and $A$, simultaneously strongly continuous in $t$ and $s$, such that

(1) $B(t, t)=C \quad \forall t \in[a, b]$, and $\|B(t, s)\| \leqq K\|A\|, a \leqq s \leqq t \leqq b$, (where $K$ may be chosen equal to 2),

(2) the range of $B(t, s)^{\circ}$ lies in the domain of $A^{-1}$ for $a \leqq s \leqq t \leqq b$, and $A^{-1} B(\cdot, s)$ $\in L^{\infty}(B(X),[s, b])$,

(3) $B(\cdot, s)$ is absolutely continuous on $[s, b]$, and $B(\cdot, s) x$ is a.e. strongly differentiable $\forall x \in X$ with derivative $A(\cdot) B(\cdot, s) x$,

(4) $B(t, \cdot)$ is absolutely continuous on $[a, t]$, and $B(t, \cdot) x$ is a.e. strongly differentiable $\forall x \in X$ with derivative

$$
-\overline{B(t, \cdot) A(\cdot) x} \quad\left(-\overline{B(t, s) A(s)}=-A^{-1} A \overline{B(t, s) A(s)}=-A^{-1} B(t, s) A(s) A\right),
$$


(5) the range of $B(t, s) C$ lies in the domain of $C^{-1}$, and $B(t, s) C=$ $B\left(t, s_{0}\right) C^{-1} B\left(s_{0}, s\right) C$ for $s \leqq s_{0} \leqq t \leqq b$.

$B(\cdot, s) \in L^{\infty}(B(X),[s, b])$ is uniquely characterized by properties (1), (2), and (3).

Proof. Let us construct $C_{1}$. Let $\left\{E_{\lambda}\right\}$ be the spectral family of $A$. Set $P_{n}=E_{1 / n}^{\perp}$. Since the range of $A$ lies in the domain of every $A(t)$, the range of $A\left(A^{-1} P_{n}\right)=P_{n}$ lies in the domain of every $A(t)$, and

$$
\left\|A(\cdot) P_{n}\right\|=\left\|A(\cdot) A\left(A^{-1} P_{n}\right)\right\| \leqq\|A(\cdot) A\|\left\|A^{-1} P_{n}\right\| \leqq n\|A(\cdot) A\|,
$$

so that $A(\cdot) P_{n} \in L^{1}(B(X),[a, b]) \quad \forall n$. Letting $a_{n}=\left\|A(\cdot) P_{n}\right\|_{1}$, so that $a_{n} \uparrow$, set $C_{1}=\frac{1}{2} e^{-a_{1}} P_{1}+\sum_{n=2}^{\infty} 2^{-n} e^{-a_{n}}\left(P_{n}-P_{n-1}\right)$, set $C_{2}=A$, and set $C=C_{1} C_{2}=C_{1} A$.

For the $A_{n}(\cdot)$ of Theorem 1 let us take $A(\cdot) P_{n}$. Since $B_{n}(t, s)$ is a uniform limit of sums of strong integrals of operators in $\mathscr{A}^{\prime}, B_{n}(t, s) \in \mathscr{A}^{\prime}$, so that $B_{n}(t, s) C_{1} C_{2}$ $=B_{n}(t, s) C_{1} A=A B_{n}(t, s) C_{1}$ has range in the domain of $A^{-1}$, and $A^{-1} B_{n}(t, s) C_{1} A$ $=B_{n}(t, s) C_{1}$. We have shown hypothesis (1) of Theorem 1 to hold and will have shown hypothesis (2) also to hold as soon as we show that $\left\|B_{n}(t, s) C_{1}\right\|$ is uniformly bounded for $a \leqq s \leqq t \leqq b, \quad \forall n$.

Consider $\left(B_{n}(t, s)-I\right) P_{m}+I$ for $m \leqq n$. We have

$$
\left(B_{n}(t, s)-I\right) P_{m} x=\int_{s}^{t} A(r) P_{n} B_{n}(r, s) P_{m} x d r=\int_{s}^{t} A(r) P_{m} B_{n}(r, s) P_{m} x d r,
$$

so that $\left(B_{n}(t, s)-I\right) P_{m}+I=B_{m}(t, s)$ by uniqueness. Thus

$$
\begin{aligned}
\left\|B_{n}(t, s) C_{1}\right\| & \leqq\left\|\left(B_{n}(t, s)-I\right) C_{1}\right\|+\left\|C_{1}\right\| \leqq\left\|\left(B_{n}(t, s)-I\right) P_{n} C_{1}\right\|+\frac{1}{2} \\
& \leqq\left\|\left(B_{n}(t, s)-I\right) P_{1} C_{1}\right\|+\sum_{k=2}^{n}\left\|\left(B_{n}(t, s)-I\right)\left(P_{k}-P_{k-1}\right) C_{1}\right\|+1 \\
& \leqq\left\|\left(B_{1}(t, s)-I\right)\right\|\left\|P_{1} C_{1}\right\|+\sum_{k=2}^{n}\left\|\left(B_{n}(t, s)-I\right) P_{k}\right\|\left\|\left(P_{k}-P_{k-1}\right) C_{1}\right\|+1 \\
& \leqq \sum_{k=1}^{n} 2^{-k} e^{-a_{k}}\left\|B_{k}(t, s)-I\right\|+1 \leqq \sum_{k=1}^{n} 2^{-k} e^{-a_{k}}\left(e^{a_{k}}-1\right)+1 \leqq 2 .
\end{aligned}
$$

For hypothesis (3) of Theorem 1 we have $\left\|A_{n}(\cdot) A\right\| \leqq\left\|A(\cdot) P_{n} A\right\|=\left\|A(\cdot) A P_{n}\right\|$ $\leqq\|A(\cdot) A\|$, and we need only choose $k(\cdot) \in L^{1}([a, b])=2\|A(\cdot) A\|$.

Finally, $\left(A_{n}(t) A\right)^{*} \rightarrow(A(t) A)^{*}$ strongly for each $t$. As usual, since we have just shown that $\left\|\left(A_{n}(\cdot) A\right)^{*}\right\|=\left\|A_{n}(\cdot) A\right\| \leqq\|A(\cdot) A\|$, we need only show strong convergence for $x$ in the range of some $P_{k}$. But then for $n \geqq k, A A_{n}(t)^{*} x=A A(t)^{*} P_{n} x=$ $A A(t) * x$.

To verify the hypotheses of Theorems 2 and 3 with $C_{3}=C_{1}$, we need only show that $A_{n}(t) A \rightarrow A(t) A$ strongly, and, again, for $x$ in the range of $P_{k}$, when $n \geqq k$ we have $A_{n}(t) A x=A(t) P_{n} A x=A(t) A P_{n} x=A(t) A x$.

Now if $\tilde{B}(t, s)$ is the relevant solution according to Theorem 1 , it is in $\mathscr{A}^{\prime}$ since it is a weak limit of operators $B_{n}(t, s) C_{1}$. Setting $B(t, s)=\widetilde{B}(t, s) A$, we may immediately verify all the assertions of the present theorem except joint strong 
continuity of $B(t, s)$ in $t$ and $s$. But this is immediate in the usual way: assuming $\delta$ and $\delta^{\prime}>0$ and small,

$$
\begin{aligned}
& \left\|\left(B\left(t+\delta, s+\delta^{\prime}\right)-B(t, s)\right) C x\right\| \\
& \quad \leqq\|(B(t+\delta, s)-B(t, s)) C x\|+\left\|B\left(t+\delta, s+\delta^{\prime}\right) C^{-1}\left(B\left(s+\delta^{\prime}, s\right)-I\right) C x\right\| \\
& \quad \leqq\|(B(t+\delta, s)-B(t, s)) C x\|+\left\|B\left(t+\delta, s+\delta^{\prime}\right)\right\|\left\|\left(B\left(s+\delta^{\prime}, s\right)-I\right) x\right\| .
\end{aligned}
$$

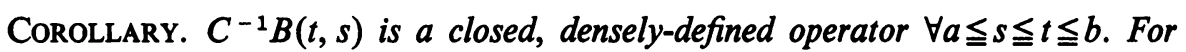
elements $x$ in the range of $C, C^{-1} B(\cdot, s) x$ and $C^{-1} B(t, \cdot) x$ are absolutely continuous on $[s, b]$ and $[a, t]$, respectively, and they are a.e. strongly differentiable with derivatives $A(\cdot) C^{-1} B(\cdot, s) x$ and $-C^{-1} B(t, \cdot)(A(\cdot) x)$. If $B_{1}(t, s)$ is related to $-A(\cdot)^{*}$ in the same way that $B(t, s)$ is related to $A(\cdot), C$ may be chosen the same for $B$ and $B_{1}$. $B_{1}(\cdot, s)^{*}$ and $B_{1}(t, \cdot)^{*}$ are absolutely continuous on $[s, b]$ and $[a, t]$, respectively, and $B_{1}(\cdot, s)^{*} x$ and $B_{1}(t, \cdot)^{*} x$ are a.e. strongly differentiable $\forall x \in X$ with derivatives $\left[-B_{1}(\cdot, s)^{*} A(\cdot)\right]^{-} x$ and $A(\cdot) B_{1}(t, \cdot)^{*} x . C^{-1} B_{1}(t, s)^{*}$ is a closed, densely-defined operator, and

$$
\overline{\left(C^{-1} B_{1}(t, s)^{*}\right)\left(C^{-1} B(t, s)\right)}=I,
$$

and

$$
\overline{\left(C^{-1} B(t, s)\right)\left(C^{-1} B_{1}(t, s)^{*}\right)}=I .
$$

Proof. Only the last statement is not immediate. But $C^{-1} B_{1}(t, s)^{*} C^{-1} B(t, s) C^{2} x$ $=B_{1}(t, s)^{*} B(t, s) x$. By taking derivatives in $t, B_{1}(t, s)^{*} B(t, s) x=B_{1}(s, s)^{*} B(s, s) x$ $=C^{2} x \quad \forall t \in[s, b]$, so that $\left(C^{-1} B_{1}(t, s)^{*}\right)\left(C^{-1} B(t, s)\right)$ is the identity on the range of $C^{2}$.

A similar computation taking derivatives in $s$ for

$$
B(t, s) B_{1}(t, s)^{*} x=C^{-1} B(t, s) C^{-1} B_{1}(t, s)^{*} C^{2} x
$$

shows that $\left(C^{-1} B(t, s)\right)\left(C^{-1} B_{1}(t, s)^{*}\right)$ is the identity on the range of $C^{2}$ as well.

4. The Cauchy problem for measurable operators affiliated with a weakly-closed ring of operators in a separable Hilbert space. (a) Some observations on measurable functions from a measure space $\Omega$ to $B(X)$ when $X$ is a separable Hilbert space are in order here.

For $T \in B(X)$, let us define $|T|$ by the polar decomposition $T=U|T|$. Unless otherwise indicated, let us assume all spectral families of projections $\left\{E_{\lambda}\right\}$ to be normalized from above from now on, so that $E_{\lambda_{0}}=\bigcap_{\lambda>\lambda_{0}} E_{\lambda_{1}}$.

Suppose now that $A(\cdot) \in \mathscr{M}(B(X), \Omega)$, where each $A(t)$ is positive self-adjoint with spectral family $\left\{E_{\lambda}(t)\right\}$. Suppose first that $\|A(\cdot)\|<M$ a.e. If $f$ is any continuous function on $[0, M]$, there exists a sequence $\left\{p_{n}\right\}$ of polynomials converging uniformly to $f$. Thus $\left(p_{n}(A(\cdot)) x, y\right) \rightarrow(f(A(\cdot)) x, y)$ pointwise, so that $f(A(\cdot)) \in$ $\mathscr{M}(B(X), \Omega)$. If $\lambda<M$, set $f_{n}(x)=1$ for $0 \leqq x \leqq \lambda,=n(1 / n+\lambda-x)$ for $\lambda \leqq x \leqq \lambda+1 / n$, 
$=0$ for $\lambda+1 / n \leqq x \leqq M$. Then $\left(f_{n}(A(\cdot)) x, y\right) \rightarrow\left(E_{\lambda}(\cdot) x, y\right)$ pointwise, so that $E_{\lambda}(\cdot) \in \mathscr{M}(B(X), \Omega)$. Since $\|A(\cdot)\|$ is in general measurable, $E_{\lambda}(\cdot) \in \mathscr{M}(B(X), \Omega)$ generally by splitting up $\Omega$ into a countable number of pieces on which $\|A(\cdot)\|$ is uniformly bounded.

Let $A(\cdot) \in \mathscr{M}(B(X), \Omega)$ be arbitrary, $=U(\cdot)|A(\cdot)|$. Then $A(\cdot)^{*} A(\cdot) \in \mathscr{M}(B(X), \Omega)$, so that $\left(A(\cdot)^{*} A(\cdot)\right)^{1 / 2}=|A(\cdot)| \in \mathscr{M}(B(X), \Omega)$. If $\left\{E_{\lambda}(t)\right\}$ is the spectral family for $|A(t)|, \int_{1 / n}^{n}(1 / \lambda) d E_{\lambda}(\cdot)$ is measurable, so that

$$
U(\cdot)|A(\cdot)| \int_{1 / n}^{n}(1 / \lambda) d E_{\lambda}(\cdot)=U(\cdot)\left(E_{n}^{-}(\cdot)-E_{1 / n}(\cdot)\right),
$$

and, ultimately, $U(\cdot) \in \mathscr{M}(B(X), \Omega)$.

In general let us make the following definition.

Definition. Let $A(\cdot)$ be a function from the measure space $\Omega$ to $C D(X)$, where $X$ is a separable Hilbert space. Let $A(\cdot)=U(\cdot)|A(\cdot)|$ be the polar decomposition for $A(\cdot)$, and let $\left\{E_{\lambda}(\cdot)\right\}$ be the spectral family of $|A(\cdot)|$. Then $A(\cdot)$ will be called measurable on $\Omega$ if $A(\cdot) E_{\lambda}(\cdot)$ is measurable on $\Omega \quad \forall \lambda \geqq 0$.

Note that $A(\cdot)$ measurable implies that $A(\cdot)^{*}$ is measurable since $A(\cdot)^{*}=$ $U(\cdot)^{*}\left(U(\cdot)|A(\cdot)| U(\cdot)^{*}\right)$, and the spectral family for $U(\cdot)|A(\cdot)| U(\cdot)^{*}$ is $U(\cdot) E_{\lambda}(\cdot) U(\cdot)^{*}$, so that

$$
\begin{aligned}
A(\cdot)^{*}\left(U(\cdot) E_{\lambda}(\cdot) U(\cdot)^{*}\right) & =U(\cdot)^{*}\left(U(\cdot) E_{\lambda}(\cdot) U(\cdot)^{*}\right)\left(U(\cdot)|A(\cdot)| U\left(\cdot \dot{j}^{*}\right)\right. \\
& =E_{\lambda}(\cdot)|A(\cdot)| U(\cdot)^{*} \\
& =\left(U(\cdot)|A(\cdot)| E_{\lambda}(\cdot)\right)^{*} \in \mathscr{M}(B(X), \Omega) .
\end{aligned}
$$

Note also that $U(\cdot) E_{n}(\cdot) \in \mathscr{M}(B(X), \Omega) \quad \forall n \geqq 0$, so that $U(\cdot) \in \mathscr{M}(B(X), \Omega)$. Consider now $P(\cdot) \in \mathscr{M}(B(X), \Omega)$, where each $P(t)$ is a projection. Since $\left(\left(P_{1}(\cdot) P_{2}(\cdot)\right)^{n} x, y\right) \rightarrow\left(P_{1}(\cdot) \cap P_{2}(\cdot) x, y\right), P_{1}(\cdot) \cap P_{2}(\cdot) \in \mathscr{M}(B(X), \Omega)$. Also,

$$
P_{1}(\cdot) \cup P_{2}(\cdot)=1-\left(1-P_{1}(\cdot)\right) \cap\left(1-P_{2}(\cdot)\right),
$$

so that $P_{1}(\cdot) \cup P_{2}(\cdot)$ is measurable. Thus $\bigcup_{i=1}^{\infty} P_{i}(\cdot) \in \mathscr{M}(B(X), \Omega)$.

[If $\mathscr{A}$ is a weakly-closed ring of operators in $X$, and if $P$ is a projection in $\mathscr{A}$, then $P^{\prime}$, the central support of $P,=\bigcup_{i=1}^{\infty} U_{i} P U_{i}^{-1}$, where $\left\{U_{i}\right\}$ ranges over a countable set of unitaries dense in the separable metric space of all unitaries of $\mathscr{A}$ under the strong topology. For if $x$ is in the range of $P$ and if $U$ is any unitary in $\mathscr{A}$, there exists a sequence $\left\{U_{i_{k}}\right\}$ with $U_{i_{k}} x \rightarrow U x$, which means that $\bigcup_{i=1}^{\infty} U_{i} P U_{i}^{-1}$ contains all images of elements in the range of $P$ under unitaries in $\mathscr{A}$, which means that it stable under all unitaries of $\mathscr{A}$, which means that it is in $\mathscr{A}$, which means that it is in the center of $\mathscr{A}$.

Then if $P(\cdot) \in \mathscr{M}(B(X), \Omega)$, with each $P(t)$ a projection in $\mathscr{A}, P^{\prime}(\cdot) \in \mathscr{M}(B(X), \Omega)$.

Suppose $A \in \mathscr{A}^{+}$, with $A=\int_{0}^{M} \lambda d E_{\lambda}$. Set $\tilde{E}_{\lambda}^{+}$equal to the central support of $\left(1-E_{\lambda}\right)$. Then $\left\{E_{\lambda}\right\}$ is a spectral family continuous from above. For $E_{\lambda_{0}}=\bigcap_{\lambda_{\lambda}>\lambda_{0}} E_{\lambda}$ 
is equivalent to $\widetilde{E}_{\lambda_{0}}^{\perp}=\bigcup_{\lambda>\lambda_{0}} \tilde{E}_{\lambda}^{\perp}$. Set $\tilde{A}=\int_{0}^{M} \lambda d \widetilde{E}_{\lambda}$. Since $\tilde{E}_{\lambda}^{\perp}$ is the central support of $E_{\lambda}^{\perp}, \widetilde{E}_{\lambda} \leqq E_{\lambda}$. Then $\forall x \in X,\left(\tilde{E}_{\lambda} x, x\right) \leqq\left(E_{\lambda} x, x\right)$. But then

$$
\begin{aligned}
(\tilde{A} x, x) & =\int_{0}^{M} \lambda d\left(\tilde{E}_{\lambda} x, x\right)=M(x, x)-\int_{0}^{M}\left(\tilde{E}_{\lambda} x, x\right) d \lambda \\
& \geqq M(x, x)-\int_{0}^{M}\left(E_{\lambda} x, x\right) d \lambda=\int_{0}^{M} \lambda d\left(E_{\lambda} x, x\right)=(A x, x) .
\end{aligned}
$$

If $A^{\prime}$ is any central operator in $\mathscr{A}$ with $A^{\prime} \geqq A$,

$$
A^{\prime n}-A^{n}=\left(\left(A^{\prime}-A\right)^{1 / 2}\left(A^{\prime n-1}+A^{\prime n-2} A+\cdots+A^{\prime} A^{n-2}+A^{n-1}\right)^{1 / 2}\right)^{2} \geqq 0,
$$

so that $A^{\prime n} \geqq A^{n} \quad \forall n \geqq 0$. Then if $A^{\prime}=\int_{0}^{M} \lambda d E_{\lambda}^{\prime}$ and if $x$ is a unit vector in the range of $E_{\lambda_{0}}^{\prime},\left(A^{n} x, x\right) \leqq\left(A^{\prime n} x, x\right) \leqq \lambda_{0}^{n} \quad \forall n \geqq 0$, so that $\int_{0}^{M}\left(\lambda / \lambda_{0}\right)^{n} d\left(E_{\lambda} x, x\right) \leqq 1 \quad \forall n \geqq 0$, which means $\left(E_{\lambda_{0}} x, x\right)=1$, which means $x$ is in the range of $E_{\lambda_{0}}$, which means $E_{\lambda_{0}}^{\prime} \leqq E_{\lambda_{0}}$, which means $E_{\lambda_{0}}^{\prime \perp} \geqq E_{\lambda_{0}}^{\perp}$, which means $E_{\lambda_{0}}^{\prime \perp} \geqq \tilde{E}_{\lambda_{0}}^{\perp}$, which means $E_{\lambda_{0}}^{\prime} \leqq \tilde{E}_{\lambda_{0}}$, which means $\left(A^{\prime} x, x\right) \geqq(\tilde{A} x, x)$, just as above, which means $A^{\prime} \geqq \tilde{A}$, so that $\tilde{A}$ is the least central operator in $\mathscr{A}$ majorizing $A$.

If $A(\cdot) \in \mathscr{M}(B(X), \Omega)$ with each $A(t) \in \mathscr{A}^{+}$, then $\tilde{A}(\cdot) \in \mathscr{M}(B(X), \Omega)$ since the associated $\widetilde{E}_{\lambda}(\cdot) \in \mathscr{M}(B(X), \Omega) \quad \forall \lambda \geqq 0$.]

(b) Let $\mathscr{M}(\mathscr{A})$ be the *-algebra of measurable operators affiliated with $\mathscr{A}$. We shall determine some ring-theoretic conditions for solution of the Cauchy problem for measurable functions on $[a, b]$ with values in $\mathscr{M}(\mathscr{A})$.

If $\operatorname{tr}$ is any trace on the ring $\mathscr{A}$, there exists a sequence of vectors $\left\{x_{n}\right\}$ such that $\operatorname{tr} A=\sum_{i=1}^{\infty}\left(A x_{i}, x_{i}\right) \quad \forall A \in \mathscr{A}^{+}$. Thus if $A(\cdot)$ is a measurable function on a measure space $\Omega$ with values in $\mathscr{M}(\mathscr{A})$, we have

$$
\operatorname{tr}|A(\cdot)|=\lim _{k_{\uparrow} \infty} \operatorname{tr}\left(|A(\cdot)| E_{k}(\cdot)\right)=\lim _{k \uparrow \infty} \sum_{i=1}^{\infty}\left(|A(\cdot)| E_{k}(\cdot) x_{i}, x_{i}\right),
$$

so that $\operatorname{tr}|A(\cdot)|$ is measurable.

We may now give some ring-theoretic conditions for solution of the Cauchy problem. The essential details of the construction amount to a direct generalization of the basic propositions for bounded operators. We first prove a lemma.

LEMMA. Let $\Omega$ be a finite measure space for which $L^{2}(\Omega)$ is separable. Let $\mathscr{A}$ be the multiplication ring of $L^{2}(\Omega)$. Then if $A(\cdot)$ is a measurable function from a finite measure space $\Omega^{\prime}$ to operators affiliated with $\mathscr{A}$, there exists a measurable function $k\left(\omega, \omega^{\prime}\right)$ on $\Omega \times \Omega^{\prime}$ such that for almost all $\omega^{\prime} \in \Omega^{\prime}, A\left(\omega^{\prime}\right)$ is multiplication by $k\left(\omega, \omega^{\prime}\right)$.

Proof. Let $\left\{E_{\lambda}(\cdot)\right\}$ be the spectral family associated with $|A(\cdot)|$. Set $A_{1:}(\cdot)$ $=A(\cdot) E_{n}(\cdot)$. Then if $v \in L^{2}(\Omega)$ is the function constantly equal to one, $A_{n}(\cdot) v$ $\in L^{\infty}\left(L^{2}(\Omega), \Omega^{\prime}\right) \subset L^{2}\left(L^{2}(\Omega), \Omega^{\prime}\right)$.

Thus there exists a sequence $\left\{v_{n}^{j}(\cdot)\right\}$ of countably-valued measurable functions 
from $\Omega^{\prime}$ to $L^{2}(\Omega)$ such that $\left\|A_{n}(\cdot) v-v_{n}^{j}(\cdot)\right\| \leqq 1 / j$ a.e. on $\Omega^{\prime}$ (the norm being the norm in $\left.L^{2}(\Omega)\right)$. Certainly $v_{n}^{j}\left(\omega^{\prime}\right)(\omega)=k_{n}^{j}\left(\omega, \omega^{\prime}\right)$ is measurable on $\Omega \times \Omega^{\prime}$. Also,

$$
\begin{aligned}
\int_{\Omega \times \Omega^{\prime}}\left|k_{n}^{i}\left(\omega, \omega^{\prime}\right)-k_{n}^{j}\left(\omega, \omega^{\prime}\right)\right|^{2} d \omega d \omega^{\prime} & \\
& \leqq \int_{\Omega^{\prime}} 2\left(1 / i^{2}+1 / j^{2}\right) d \omega^{\prime}=2\left(1 / i^{2}+1 / j^{2}\right) \int_{\Omega^{\prime}} d \omega^{\prime},
\end{aligned}
$$

so that $\left\{k_{n}^{\prime}\left(\omega, \omega^{\prime}\right)\right\}_{j=1}^{\infty}$ is Cauchy in $L^{2}\left(\Omega \times \Omega^{\prime}\right)$. Let $k_{n}\left(\omega, \omega^{\prime}\right) \in L^{2}\left(\Omega \times \Omega^{\prime}\right)$ be a limit function. Then $k_{n}\left(\omega, \omega^{\prime}\right)=A_{n}\left(\omega^{\prime}\right) v$ (in $L^{2}(\Omega)$, and, hence, in $L^{\infty}(\Omega)$ ) for almost all $\omega^{\prime} \in \Omega^{\prime}$ since

$$
\int_{\Omega^{\prime}} d \omega^{\prime}\left(\int_{\Omega}\left|k_{n}\left(\omega, \omega^{\prime}\right)-k_{n}^{j}\left(\omega, \omega^{\prime}\right)\right|^{2} d \omega\right) \rightarrow 0
$$

so that

$$
\int_{\Omega}\left|k_{n}\left(\omega, \omega^{\prime}\right)-k_{n}^{s} m\left(\omega, \omega^{\prime}\right)\right|^{2} d \omega \rightarrow 0
$$

for almost all $\omega^{\prime}$ for an appropriate subsequence $\left\{j_{m}\right\}$. Thus for almost all $\omega^{\prime} \in \Omega^{\prime}$, $A_{n}\left(\omega^{\prime}\right)$ is multiplication by $k_{n}\left(\omega, \omega^{\prime}\right)$.

Application of this to $E_{n}(\cdot)$ gives a function $p_{n}\left(\omega, \omega^{\prime}\right)$, which, since it is equal to 0 or 1 a.e. on $\Omega \times \Omega^{\prime}$, must be the characteristic function of a measurable set $G_{n}$. Clearly $k_{m} \chi_{G_{n}}=k_{n}$ almost everywhere $\forall m \geqq n$, so that, except on a null set of $G_{n}, k_{m}$ remains constant when $m \geqq n$. Then $\left\{k_{n}\right\}$ converges to a measurable function $k$ a.e. on $\Omega \times \Omega^{\prime}$. Since $k \chi_{G_{n}}=k_{n}, A\left(\omega^{\prime}\right)$ is multiplication by $k\left(\omega, \omega^{\prime}\right)$ for almost all $\omega^{\prime} \in \Omega^{\prime}$.

THEOREM. Let $\mathscr{A}$ be a discrete ring, and let $A_{\alpha}(\cdot)$ be a measurable function from $[a, b]$ to $\mathscr{M}(\mathscr{A}), \alpha=1,2$. Let $A_{1}(\cdot) \in L^{1}(B(X),[a, b])$, and let $\left[\operatorname{tr} A_{2}(\cdot)^{*} A_{2}(\cdot)\right]^{1 / 2}$ $\in L^{1}([a, b])$, where $\operatorname{tr}$ is an essential trace on $\mathscr{A}$. Set $A_{0}(\cdot)=A_{1}(\cdot)+A_{2}(\cdot)$. Then $\exists B(t, s) \in \mathscr{M}(\mathscr{A}) \quad \forall a \leqq s \leqq t \leqq b$ with the following properties:

(1) $B(t, t)=I \quad \forall t \in[a, b]$,

(2) there exists a central $A \in \mathscr{A}^{+} \cap L(X)$ whose range is in the domain of every $B(t, s)$, and which has $B(\cdot, s) A$ in $L^{\infty}(B(X),[s, b]) \quad \forall s \in[a, b]$ (indeed, $B(t, s) A$ is uniformly bounded over all $a \leqq s \leqq t \leqq b)$,

(3) if $x \in X$ has $(T x, x) \leqq k(x)$ tr $T \quad \forall T \in \mathscr{A}^{+}, B(\cdot, s) A x$ is absolutely continuous on $[s, b]$ and a.e. strongly differentiable on $[s, b]$ with derivative $A_{0}(\cdot) B(\cdot, s) A x($ such $x$ are dense in $X$ ),

(4) for $x$ as above, $B(t, \cdot) A x$ is absolutely continuous on $[a, t]$ and a.e. strongly differentiable on $[a, t]$ with derivative $-B(t, \cdot) A A_{0}(\cdot) x$,

(5) if $\bar{B}(t, s)$ is related to $-A_{0}(\cdot)^{*}$ as $B(t, s)$ is related to $A_{0}(\cdot), \bar{B}(t, s)$ has all of the above properties with respect to $-A_{0}(\cdot)^{*}$ and the same $A$, and $\bar{B}(t, s)^{*}=B(t, s)^{-1}$,

(6) $\forall s_{0} \in[s, t], B\left(t, s_{0}\right) B\left(s_{0}, s\right)=B(t, s)$,

(7) $B(t, s) A$ is jointly strongly continuous in $t$ and $s$.

Properties (1), (2), and (3) uniquely characterize $B(t, s)$. Further, $\forall a \leqq s \leqq t \leqq b$, $\int_{a}^{t}\left|A_{0}(r)\right|^{\sim} E_{n}(r) d r$ is a monotone increasing sequence of central operators in $\mathscr{A}$ 
converging a.e. to an unbounded positive central operator $\int_{s}^{t}\left|A_{0}(\tilde{r})\right| d r$ affiliated with $\mathscr{A}$, and $|B(t, s)-I|+I \leqq \exp \left(\int_{s}^{t}\left|A_{0}(\tilde{r})\right| d r\right) .\left(\left\{E_{\lambda}(r)\right\}\right.$ is the spectral family for $\left|A_{0}(r)\right|^{\sim}$.)

Proof. Suppose $\left\{P_{i}\right\}$ is a sequence of mutually orthogonal central projections in $\mathscr{A}$ with $\sum_{i=1}^{\infty} P_{i}=I$. Set $X_{i}=P_{i} X$, set $\mathscr{A}_{i}=\mathscr{A} P_{i}$, and set $\operatorname{tr}_{i} T=\operatorname{tr} T \quad \forall T \in \mathscr{A}_{i}^{+}$. Then $\left[\operatorname{tr}_{i} A_{2}(\cdot)^{*} A_{2}(\cdot) P_{i}\right]^{1 / 2} \in L^{1}([a, b])$.

Suppose that all of the assertions of the theorem hold for $A_{1}(\cdot) P_{i}+A_{2}(\cdot) P_{i}$. Let $B_{i}(t, s)$ and $A_{t}$ be the relevant " $B(t, s)$ " and " $A$ ". Set

$$
a_{i}=\sup _{a \leqq s \leqq t \leqq b}\left\{\left\|\bar{B}_{i}(t, s) A\right\|,\left\|B_{i}(t, s) A\right\|\right\},
$$

and let $A=\sum_{i=1}^{\infty} a_{i}^{-1} A_{i}$. Let $B(t, s)=\sum_{i=1}^{\infty} B_{i}(t, s)$.

Then assertions (1) and (2) certainly hold. For assertion (3), let $x \in X$ be such that $(T x, x) \leqq k(x) \operatorname{tr} T \quad \forall T \in \mathscr{A}^{+}$. We have $\forall t \in[a, b]$,

$$
\operatorname{tr}\left(A B(t, s)^{*} A_{2}(t)^{*} A_{2}(t) B(t, s) A\right) \leqq K^{2} \operatorname{tr} A_{2}(t)^{*} A_{2}(t)
$$

( $K^{2}$ is independent of $s$ and $t$ ), so that $\left\|A_{2}(t) B(t, s) A x\right\|^{2} \leqq k(x) K^{2} \operatorname{tr} A_{2}(t)^{*} A_{2}(t)$, which means $\left\|A_{2}(\cdot) B(\cdot, s) A x\right\| \in L^{1}([s, b])$, and, therefore, $\left(A_{1}(\cdot)+A_{2}(\cdot)\right) B(\cdot, s) A x$ $\in L^{1}(B(X),[s, b])$. Thus $\int_{s}^{t} A_{0}(r) B(r, s) A x d r$ exists, and we have only to show that it is equal to $B(t, s) A x-A x$. But this is immediate upon application of $P_{i}$.

Assertion (4) follows in exactly the same manner. For assertion (5) we need only take $\bar{B}(t, s)=\sum_{i=1}^{\infty} \bar{B}_{i}(t, s)$. Assertion (6) is immediate. Assertion (7) follows from joint strong continuity on elements in the range of $\sum_{i=1}^{n} P_{i}$ and uniform boundedness of $B(t, s) A$.

Uniqueness follows by application of $P_{i}$ and the observation that properties (1), (2), and (3) are inherited when everything is reduced to $\mathscr{A}_{i}$. The operator bound is immediate.

Since $\mathscr{A}$ is discrete and $X$ is separable, there exists a sequence of mutually orthogonal central projections $\left\{P_{n}\right\}$ such that each $P_{n}$ is the sum of $n$ orthogonal equivalent abelian projections, $n=1,2, \ldots, \infty$. By the above discussion we may assume that some $P_{n}=I$.

We assume then that there exists a sequence $\left\{P_{t}\right\}_{i=1}^{n}$ of mutually orthogonal equivalent abelian projections with $\sum_{i=1}^{n} P_{i}=I$. Let $U_{i j} \in \mathscr{A}$ be a partial isometry with $U_{i j}^{*}=U_{j i}$, and $U_{i j} U_{j i}=P_{i}$. Since $P_{1} \mathscr{A} P_{1}$ is abelian, it is isomorphic to the multiplication algebra $\mathscr{M}$ of $L^{\infty}(\Omega)$ over $L^{2}(\Omega)$, where $\Omega$ is a finite measure space with $L^{2}(\Omega)$ separable. The integral of a function in $L^{\infty}(\Omega)$ gives a trace $\operatorname{tr}_{1}$ on $P_{1} \mathscr{A} P_{1}$. If we set $\operatorname{tr}^{\prime} T=\sum_{j=1}^{n} \operatorname{tr}_{1}\left(U_{1 j} T U_{j 1}\right) \forall T \in \mathscr{A}^{+}, \operatorname{tr}^{\prime}$ is clearly additive, homogeneous with respect to nonnegative real numbers, and normal. For all $T \in \mathscr{A}$,

$$
\begin{aligned}
\operatorname{tr}^{\prime} T^{*} T & =\sum_{j=1}^{n} \operatorname{tr}_{1}\left(U_{1 j} T^{*} T U_{j 1}\right)=\sum_{j, k=1}^{n} \operatorname{tr}_{1}\left(\left(U_{1 j} T^{*} U_{k 1}\right)\left(U_{1 k} T U_{j 1}\right)\right) \\
& =\sum_{j, k=1}^{n} \operatorname{tr}_{1}\left(\left(U_{1 k} T U_{j 1}\right)\left(U_{1 j} T^{*} U_{k 1}\right)\right)=\sum_{k=1}^{n} \operatorname{tr}_{1}\left(U_{1 k} T T^{*} U_{k 1}\right)=\operatorname{tr}^{\prime} T T^{*},
\end{aligned}
$$


so that $\operatorname{tr}^{\prime}$ is a trace. Since for $T \in \mathscr{A}^{+}, \operatorname{tr}^{\prime} T=0$ if and only if $P_{i} T P_{i}=0 \quad \forall i$, if and only if $\left\|T^{1 / 2} P_{2} x\right\|^{2}=0 \quad \forall x \in X$, if and only if $T=0, \operatorname{tr}^{\prime}$ is essential.

Let $S$ be an unbounded 1-1 positive operator affiliated with the center of $\mathscr{A}$ such that $\operatorname{tr} T=\operatorname{tr}^{\prime} S T \quad \forall T \in \mathscr{A}^{+}$. Let $S$ have spectral resolution $\left\{E_{\lambda}\right\}$, and set $Q_{k}=E_{2^{k}}-E_{2^{k-1}}$. Then $\left\{Q_{k}\right\}$ is a sequence of mutually orthogonal central projections with $\sum_{-\infty}^{\infty} Q_{k}=I$, and $\operatorname{tr} T Q_{k}=\operatorname{tr}^{\prime} S Q_{k} T \leqq 2^{k} \operatorname{tr}^{\prime} T Q_{k}, \operatorname{tr}^{\prime} T Q_{k}=\operatorname{tr} T S^{-1} Q_{k}$ $\leqq 2^{1-k} \operatorname{tr} T Q_{k}$. If we assume, as we may, that some $Q_{k}=I$, then we may assume the isomorphism of $P_{1} \mathscr{A} P_{1}$ with the multiplication algebra $\mathscr{M}$ of $L^{\infty}(\Omega)$ over $L^{2}(\Omega)$ to be such that $\operatorname{tr}$ gives the same measure as $\operatorname{tr}_{1}$ on $\Omega$ since $\operatorname{tr}$ and $\operatorname{tr}_{1}$ give equivalent measures inducing a unitary transformation of $L^{2}(\Omega)$. Call the isomorphism $\theta$.

Consider now $\theta\left(U_{1 i} A_{\alpha}(\cdot) U_{j 1}\right)$. This is a measurable function from $[a, b]$ to unbounded operators affiliated with $\mathscr{M}$. Thus by the lemma there exists a function $k_{i j}^{\alpha}(\omega, t)$ measurable on $\Omega \times[a, b]$ with $\theta\left(U_{1 i} A_{\alpha}(t) U_{j 1}\right)$ multiplication by $k_{i j}^{\alpha}(\cdot, t)$ for almost all $t \in[a, b]$. Further, for almost all $t, k_{i j}^{\alpha}(\omega, t)$ defines a bounded matrix operator on $l^{2}\left(\{j\}_{j=1}^{n}\right)$ with norm $\left\|k_{i j}^{\alpha}(\omega, t)\right\|_{l^{2}} \quad \forall \omega \in \Omega$ if we set $k_{i j}^{\alpha}(\omega, t)$ equal to zero on a suitable null set of $\Omega \times[a, b]$, an adjustment which changes $k_{i j}^{\alpha}(\omega, t)$ only on a null set of $\Omega$ for almost all $t$ since a null set of $\Omega \times[a, b]$ has null $t$-sections for almost all $t$ by the Fubini theorem. Indeed, the function $\kappa_{i j}^{\alpha}(\omega, t)$ for $\left|A_{\alpha}(\cdot)\right|^{\sim}$ has $\tilde{k}_{i j}^{\alpha}(\omega, t)=0$ for $i \neq j$, and $\tilde{k}_{11}^{\alpha}(\omega, t)=\tilde{k}_{i i}^{\alpha}(\omega, t) \geqq 0 \quad \forall i$. If $F$ is the set of all finite sequences of complex rationals with $l^{2}$-norm $\leqq 1$, the function

$$
\sup _{v \in F}\left\|\left(k_{i j}^{\alpha}(\omega, t)\right)(v)\right\|_{t^{2}}
$$

is measurable and $\geqq 0$ on $\Omega \times[a, b]$, and must by its properties relative to $A_{\alpha}(\cdot)$ be equal a.e. on $\Omega \times[a, b]$ to $\tilde{k}_{11}^{\alpha}(\cdot, t)$.

We have

$$
\begin{aligned}
\operatorname{tr} A_{2}(t)^{*} A_{2}(t) & =\sum_{j=1}^{n} \operatorname{tr}\left(U_{1 j} A_{2}(t)^{*} A_{2}(t) U_{j 1}\right) \\
& =\sum_{i, j=1}^{n} \operatorname{tr}\left(U_{1 j} A_{2}(t)^{*} U_{i 1} U_{1 i} A_{2}(t) U_{j 1}\right) \\
& =\int_{\Omega} \sum_{i, j=1}^{n} k_{i j}^{2}(\omega, t) k_{i j}^{2}(\omega, t) d \omega \geqq \int_{\Omega} k_{11}^{2}(\omega, t)^{2} d \omega
\end{aligned}
$$

since the Hilbert-Schmidt norm majorizes the operator norm, so that

$$
\int_{\Omega} \mathscr{k}_{11}^{2}(\omega, \cdot) d \omega \in L^{1}([a, b])
$$

and, consequently, $\int_{a}^{b} \tilde{k}_{11}^{2}(\cdot, t) d t \in L^{1}(\Omega)$ by the Fubini theorem. Set $\Omega_{m}=$ $\left\{\omega \in \Omega: m-1 \leqq \int_{a}^{b} \hat{k}_{11}(\omega, t)^{2} d t<m\right\}$, so that the $\Omega_{m}$ are disjoint and $\Omega=\bigcup_{m=1}^{\infty} \Omega_{m}$ up to a null set. Let $Q_{m}=\sum_{j=1}^{n} U_{j 1} \theta^{-1}\left(\chi_{\Omega_{m}}\right) U_{1 j}$. Then $\forall T \in \mathscr{A}$,

$$
\begin{aligned}
Q_{m} T & =\sum_{j=1}^{n} U_{j 1} \theta^{-1}\left(\chi_{\Omega_{m}}\right) U_{1 j} T=\sum_{j, k=1}^{n} U_{j 1} \theta^{-1}\left(\chi_{\Omega_{m}}\right) U_{1 j} T U_{k j} U_{j k} \\
& =\sum_{j, k=1}^{n} P_{j} T U_{k j} U_{j 1} \theta^{-1}\left(\chi_{\Omega_{m}}\right) U_{1 j} U_{j k}=T Q_{m},
\end{aligned}
$$


so that $Q_{m}$ is a central projection. Since $\sum_{m=1}^{\infty} Q_{m}=I$, we may, as twice before, assume that $Q_{m}=I$ for some $m$, so that $\int_{a}^{b} \tilde{k}_{11}^{0}(\cdot, t) d t \leqq M$ a.e. on $\Omega$.

Let $K(\omega, t)$ be the linear transformation on $l^{2}\left(\{j\}_{j=1}^{n}\right)$ defined by the matrix $k_{i j}^{0}(\omega, t)$. By the propositions for bounded operators $\exists B(\omega, t, s)$ for almost all $\omega$ and $\forall a \leqq s \leqq t \leqq b$ with $\|B(\omega, t, s)-I\|_{l^{2}}+1 \leqq \exp \left(\int_{s}^{t} k_{11}^{0}(\omega, r) d r\right) \leqq e^{M}$, and with $B(\omega, \cdot, s)$ absolutely continuous on $[s, b]$ and a.e. strongly differentiable with derivative $K(\omega, \cdot) B(\omega, \cdot, s)$.

Since $K(\omega, t)$ is measurable from $\Omega \times[a, b]$ to $B\left(l^{2}\left(\{j\}_{j=1}^{n}\right)\right)=B(H), K\left(\omega, t_{n}\right)$ $\cdots K\left(\omega, t_{1}\right)$ is measurable from $\Omega \times[a, b]^{n}$ to $B(H)$. We have by the Fubini theorem, defining $B_{n}(\omega, t, s)$ by

$$
B_{n}(\omega, t, s) h=\int_{t \geqq t_{n} \geqq \ldots \geqq t_{1} \geqq s} K\left(\omega, t_{n}\right) \cdots K\left(\omega, t_{1}\right) h d t_{1} \cdots d t_{n}
$$

for $h \in H$, that

$$
\left(B_{n}(\omega, t, s) h, h^{\prime}\right)=\int_{t \geqq t_{n} \geqq \ldots \geqq t_{1} \geqq s}\left(K\left(\omega, t_{n}\right) \cdots K\left(\omega, t_{1}\right) h, h^{\prime}\right) d t_{1} \cdots d t_{n}
$$

is measurable in $\omega, t$, and $s$. Thus $B(\omega, t, s)=I+\sum_{n=1}^{\infty} B_{n}(\omega, t, s)$ is measurable in $\omega, t$, and $s$. Set $B(t, s)=\sum_{i, j=1}^{n} U_{i 1} \theta^{-1}\left(B(\cdot, t, s)_{i j}\right) U_{1 j}$, and set $A$ of assertion (2) of the theorem equal to $I$. Certainly $\|B(t, s)\| \leqq e^{M} \quad \forall a \leqq s \leqq t \leqq b$, and $B(t, t)=I$ $\forall t \in[a, b]$. Measurability of $B(t, s)$ in $t$ and $s$ is also clear (since it may be expressed in the form " $\rho(B(t, s))$ is measurable in $t$ and $s$ for any positive normal form $\rho$ on $\mathscr{A} ")$.

Suppose $(T x, x) \leqq k(x) \operatorname{tr} T \quad \forall T \in \mathscr{A}^{+}$for some $x \in X$. Then

$$
\begin{aligned}
\left(A_{2}(\cdot) B(\cdot, s) x, A_{2}(\cdot) B(\cdot, s) x\right) & =\left(B(\cdot, s)^{*} A_{2}(\cdot)^{*} A_{2}(\cdot) B(\cdot, s) x, x\right) \\
& \leqq k(x) \operatorname{tr}\left(B(\cdot, s)^{*} A_{2}(\cdot)^{*} A_{2}(\cdot) B(\cdot, s)\right) \\
& \leqq k(x) e^{2 M} \operatorname{tr} A_{2}(\cdot)^{*} A_{2}(\cdot),
\end{aligned}
$$

so that $\left\|A_{2}(\cdot) B(\cdot, s) x\right\| \in L^{1}([s, b])$, and so

$$
A_{0}(\cdot) B(\cdot, s) x \in L^{1}(B(X),[s, b]) .
$$

We wish to show that

$$
B(t, s) x=x+\int_{s}^{t} A_{0}(r) B(r, s) x d r .
$$

Note that the set of $x \in X$ with $(T x, x) \leqq k(x) \operatorname{tr} T$, or $\left\|T^{1 / 2} x\right\|^{2} \leqq k(x) \operatorname{tr} T$, forms a linear space $\left(T \in \mathscr{A}^{+}\right)$. For

$$
\left\|T^{1 / 2} \lambda x\right\|^{2} \leqq|\lambda|^{2} k(x) \operatorname{tr} T,
$$

and

$$
\begin{aligned}
\left\|T^{1 / 2}(x+y)\right\|^{2} & \leqq\left(\left\|T^{1 / 2} x\right\|+\left\|T^{1 / 2} y\right\|\right)^{2} \leqq 2\left(\left\|T^{1 / 2} x\right\|^{2}+\left\|T^{1 / 2} y\right\|^{2}\right) \\
& \leqq 2(k(x)+k(y)) \operatorname{tr} T .
\end{aligned}
$$


Since tr $T=\sum_{i=1}^{\infty}\left(T x_{i}, x_{i}\right)$ for some separating sequence of vectors $\left\{x_{i}\right\}$, the space of $x \in X$ with $\left\|T^{1 / 2} x\right\|^{2} \leqq k(x) \operatorname{tr} T$ is a separating set for $\mathscr{A}$. For any unitary $U \in \tilde{A}$, $\left\|T^{1 / 2}(U x)\right\|^{2}=\left\|U T^{1 / 2} x\right\|^{2}=\left\|T^{1 / 2} x\right\|^{2} \leqq k(x) \operatorname{tr} T$, so that the space of $x \in X$ with $\left\|T^{1 / 2} x\right\|^{2} \leqq k(x) \operatorname{tr} T$ is dense in $X$.

Thus it suffices to show that $(B(t, s) x, y)=(x, y)+\int_{s}^{t}\left(A_{0}(r) B(r, s) x, y\right) d r \quad \forall y$ with $\left\|T^{1 / 2} y\right\|^{2} \leqq k(y) \operatorname{tr} T$. By decomposing each term in the form

$$
(x, y)=\frac{1}{4}((x+y, x+y)-(x-y, x-y)+i(x+i y, x+i y)-i(x-i y, x-i y)),
$$

we see that it suffices to show that $(B(t, s) x, x)=(x, x)+\int_{s}^{t}\left(A_{0}(r) B(r, s) x, x\right) d r$ $\forall x \in X$ with $\left\|T^{1 / 2} x\right\|^{2} \leqq k(x) \operatorname{tr} T$ for $T \in \mathscr{A}^{+}$. Since $T \rightarrow(T x, x)$ is a positive normal form on $\mathscr{A}$, it defines a positive normal form on $B(H) \otimes \mathscr{M}$, given by $T(\cdot) \rightarrow \sum_{m=1}^{\infty} \int_{\Omega}\left(T(\omega) f_{m}(\omega), f_{m}(\omega)\right) d \omega$, where $f_{m}(\omega) \in H$ for each $\omega \in \Omega$ with $\sum_{m=1}^{\infty} \int_{\Omega}\left\|f_{m}(\omega)\right\|^{2} d \omega<\infty$, and where $T(\omega) \in B(H)$ for each $\omega$ is defined by the matrix $T_{i j}(\cdot)=\theta\left(U_{1 i} T U_{j 1}\right)$. Since

$$
\left(B(\omega, t, s) f_{m}(\omega), f_{m}(\omega)\right)=\left(f_{m}(\omega), f_{m}(\omega)\right)+\int_{s}^{t}\left(K(\omega, r) B(\omega, r, s) f_{m}(\omega), f_{m}(\omega)\right) d r
$$

for almost all $\omega \in \Omega$, we have

$$
\begin{aligned}
\int_{\Omega}\left(B(\omega, t, s) f_{m}(\omega), f_{m}(\omega)\right) d \omega= & \int_{\Omega}\left\|f_{m}(\omega)\right\|^{2} d \omega \\
& +\int_{s}^{t} d r \int_{\Omega}\left(K(\omega, r) B(\omega, r, s) f_{m}(\omega), f_{m}(\omega)\right) d \omega
\end{aligned}
$$

by the Fubini theorem because

$$
\begin{aligned}
\int_{s}^{t} d r & \int_{\Omega}\left|\left(K(\omega, r) B(\omega, r, s) f_{m}(\omega), f_{m}(\omega)\right)\right| d \omega \\
& \leqq e^{M} \int_{s}^{t} d r \int_{\Omega}\left\|f_{m}(\omega)\right\|\left\|K(\omega, r)^{*} f_{m}(\omega)\right\| d r \\
& \leqq e^{M} \int_{s}^{t} d r\left(\int_{\Omega}\left\|f_{m}(\omega)\right\|^{2} d \omega\right)^{1 / 2}\left(\int_{\Omega}\left\|K(\omega, r)^{*} f_{m}(\omega)\right\|^{2} d \omega\right)^{1 / 2} \\
& \leqq e^{M}\left\|f_{m}\right\|_{2} \int_{s}^{t}\left(A_{0}(r) A_{0}(r)^{*} x, x\right)^{1 / 2} d r \\
& \leqq e^{M}\left\|f_{m}\right\|_{2}\left(\|x\| \int_{s}^{t}\left\|A_{1}(r)\right\| d r+k(x) \int_{s}^{t}\left(\operatorname{tr} A_{2}(r)^{*} A_{2}(r)\right)^{1 / 2} d r\right),
\end{aligned}
$$

which is finite. By the Lebesgue dominated convergence theorem

$$
\begin{aligned}
\sum_{m=1}^{\infty} \int_{\Omega}\left(B(\omega, t, s) f_{m}(\omega), f_{m}(\omega)\right) d \omega= & \sum_{m=1}^{\infty} \int_{\Omega}\left\|f_{m}(\omega)\right\|^{2} d \omega \\
& +\int_{\Omega}^{t} d r \sum_{m=1}^{\infty} \int_{\Omega}\left(K(\omega, r) B(\omega, r, s) f_{m}(\omega), f_{m}(\omega)\right) d \omega
\end{aligned}
$$


since

$$
\begin{aligned}
\sum_{m=1}^{N} \mid \int_{\Omega}(K(\omega, r) & \left.B(\omega, r, s) f_{m}(\omega), f_{m}(\omega)\right) d \omega \mid \\
& \leqq e^{M} \sum_{m=1}^{N} \int_{\Omega}\left\|f_{m}(\omega)\right\|\left\|K(\omega, r)^{*} f_{m}(\omega)\right\| d \omega \\
& \leqq e^{M} \sum_{m=1}^{N}\left(\int_{\Omega}\left\|f_{m}(\omega)\right\|^{2}\right)^{1 / 2}\left(\int_{\Omega}\left\|K(\omega, r)^{*} f_{m}(\omega)\right\|^{2}\right)^{1 / 2} \\
& \leqq e^{M}\left(\sum_{m=1}^{N} \int_{\Omega}\left\|f_{m}(\omega)\right\|^{2} d \omega\right)^{1 / 2}\left(\sum_{m=1}^{N} \int_{\Omega}\left\|K(\omega, r)^{*} f_{m}(\omega)\right\|^{2}\right)^{1 / 2} \\
& \leqq e^{M}\left(\sum_{m=1}^{\infty} \int_{\Omega}\left\|f_{m}(\omega)\right\|^{2}\right)^{1 / 2}\left(A_{0}(r) A_{0}(r)^{*} x, x\right)^{1 / 2} \\
& \leqq e^{M}(x, x)\left(\|x\|\left\|A_{1}(r)\right\|+k(x)\left(\operatorname{tr} A_{2}(r)^{*} A_{2}(r)\right)^{1 / 2}\right) \in L^{1}([a, b]) .
\end{aligned}
$$

But then $(B(t, s) x, x)=(x, x)+\int_{s}^{t}\left(A_{0}(r) B(r, s) x, x\right) d r$.

Assertion (4) is proved in exactly the same manner. Assertions (5) and (6) follow from the previous assertions and the isomorphism of $\mathscr{A}$ with $B(H) \otimes \mathscr{M}$. Assertion (7) follows from boundedness of $B(t, s)$ and absolute continuity of $B(t, s) x$ in $t$ and $s$ separately for $x$ with $(T x, x) \leqq k(x) \operatorname{tr} T$ for $T \in \mathscr{A}^{+}$, together with (6). The operator bound has already been established in the course of computation.

Only uniqueness of $B(t, s)$ remains to be shown. Suppose $C(\cdot)$ is a function from $[s, b]$ to $\mathscr{M}(\mathscr{A})$ with $C(s)=I$, with an associated central $A \in \mathscr{A}^{+} \cap L(X)$ whose range is in the domain of every $C(t)$, and which has $C(\cdot) A \in L^{\infty}(B(X),[s, b])$, and $C(\cdot) A x$ absolutely continuous and a.e. strongly differentiable on $[s, b]$ with derivative $A_{0}(\cdot) C(\cdot) A x \quad \forall x$ with $(T x, x) \leqq k(x) \operatorname{tr} T \quad \forall T \in \mathscr{A}^{+}$. (Note that for $S \in \mathscr{A}$,

$$
(T S x, S x)=\left(S^{*} T S x, x\right) \leqq k(x) \operatorname{tr} S^{*} T S \leqq\|S\|^{2} k(x) \operatorname{tr} T,
$$

so that

$$
\begin{aligned}
\left\|A_{2}(\cdot) C(\cdot) A x\right\|^{2} & =\left(A_{2}(\cdot)^{*} A_{2}(\cdot) C(\cdot) A x, C(\cdot) A x\right) \\
& \leqq\|C(\cdot) A\|^{2} k(x) \operatorname{tr} A_{2}(\cdot)^{*} A_{2}(\cdot)
\end{aligned}
$$

and thus $\left\|A_{2}(\cdot) C(\cdot) A x\right\|$ is in $L^{1}[(s, b])$.) Let $x$ and $y$ have $(T x, x)$ and $(T y, y)$ dominated by $\operatorname{tr} T \forall T \in \mathscr{A}^{+}$. Then $(C(\cdot) A x, \bar{B}(\cdot, s) y)$ is differentiable with derivative $\left(A_{0}(\cdot) C(\cdot) A x, \bar{B}(\cdot, s) y\right)+\left(C(\cdot) A x,-A_{0}(\cdot)^{*} \bar{B}(\cdot, s) y\right)=0$, so that

$$
(\bar{B}(t, s) * C(t) A x, y)=(A x, y) \quad \forall t \in[s, b],
$$

which means that $\bar{B}(t, s)^{*} C(t) A=A \quad \forall t \in[s, b]$, which means that $C(t) A=$ $B(t, s) A \quad \forall t \in[s, b]$, which means that $C(t)=B(t, s) \quad \forall t \in[s, b]$.

(c) If the ring $\mathscr{A}$ is not discrete, no theorem like the above one seems to be available. However, some kind of control over the traces of the operators involved can help in keeping domains under control. Let us give an example of an operator $A$ affiliated with a finite ring $\mathscr{A}$ for which $\sum_{k=0}^{\infty}(1 / k !) A^{k} x$ converges weakly for no nonzero $x \in X$. 
We take the ring $\mathscr{A}$ to be one of the standard examples of a finite factor of type $\mathrm{II}_{1}$. Let $C$ be the group of reals mod one. Let $H_{n} \cong L^{2}(C)$, and set $X=\oplus_{-\infty}^{\infty} H_{n}$. Let $a \in(0,1)$ be a fixed irrational number. For $f=\left\{f_{n}\right\} \in X$, let $(U f)_{n}(x)=f_{n-1}(x-a)$, so that $U$ is unitary on $X$. Let $(S f)_{n}=x f_{n}(x)$. Then the ring $\mathscr{A}$ generated by $U$ and $S$ is a factor of type $\mathrm{II}_{1}$. If $m(\cdot)$ is any measurable function on $C$, and if $(T f)_{n}(x)$ $=m(x) f_{n}(x)$ for suitable $f \in X$, then $U T=A$ is affiliated with $\mathscr{A}$. If we take $m(x)$ $=\exp \left(x^{-4}\right), A$ will furnish the example desired.

To see this, let us first note that there exist arbitrarily large integers $m$ such that the set of iterates $\{j a\}_{j=1}^{(m+2)^{2}}$ intersects any open subinterval of $[0,1)$ of length $\geqq 1 / m$. For by the box argument some $r a$ and $s a$ for $1 \leqq r<s \leqq\left(m^{\prime}+1\right)$ must fall in an interval $\left(k / m^{\prime},(k+1) / m^{\prime}\right)$, so that $0<(s-r) a<1 / m^{\prime}$ with $(s-r) \leqq m^{\prime}$, i.e., $0<r a<1 / m^{\prime}$ for some integer $r$ between 1 and $m^{\prime}$. Let $m$ be such that $1 /(m+1)$ $<r a<1 / m \leqq 1 / m^{\prime}$. Then $\{s r a\}_{s=1}^{m+1}$ intersects every open subinterval of $[0,1)$ of length $\geqq 1 / m$, and $s r \leqq(m+1) m^{\prime}<(m+1)^{2}$.

Suppose now that for some nonzero $f=\left\{f_{n}\right\} \in X, \sum_{n=0}^{N}(1 / k !) A^{k} f$ were weakly convergent as $N \rightarrow \infty$. Then $(1 / k !) A^{k} f \rightarrow 0$ weakly as $k \rightarrow \infty$, so that $(1 / k !)^{2}\left\|A^{k} f\right\|^{2}$ $\leqq$ some constant $K \quad \forall k$. Let $N$ be such that $f_{N} \neq 0$. We have $(1 / k !)^{2}\left\|\left(A^{k} f\right)_{N+k}\right\|^{2}$ $\leqq K \quad \forall k$, or

$$
(1 / k !)^{2} \int_{0}^{1}\left(\prod_{j=1}^{k} \exp (x-j a)^{-4}\right)^{2}\left|f_{N}(x-k a)\right|^{2} d x \leqq K \quad \forall k,
$$

so that

$$
(1 / k !) \inf \prod_{j=1}^{k} \exp (x-j a)^{-4} \leqq K^{\prime} \quad \forall k \quad\left(K^{\prime}=K^{1 / 2} /\left\|f_{N}\right\|\right) .
$$

Consider $k=(m+1)^{2}$, where $m$ is as described above. For any $x \in[0,1)$, some $j a \in(x-1 / m, x)$ for $j$ between 1 and $k$, so that inf $\prod_{j=1}^{k} \exp (x-j a)^{-4} \geqq e^{m^{4}}=$ $\exp \left(k^{1 / 2}-1\right)^{4} \geqq e^{k^{2} / 2}$ for $k \geqq 50$. Thus $(1 / k !) e^{k^{2} / 2} \leqq K^{\prime}$ for arbitrarily large $k$, so that $k^{-k} e^{k^{2} / 2} \leqq K^{\prime}$ for arbitrarily large $k$, or $e^{k(k / 2-\ln k)} \leqq K^{\prime}$ for arbitrarily large $k$, which is impossible.

Note that $T=|A|$ has very badly divergent trace $\left(\operatorname{tr} T=\int_{0}^{1} e^{x^{-4}} d x\right)$. If we consider only operators $A$ which are sums of bounded operators and operators whose absolute values have finite trace, exponentiation is always possible.

We preface the statement of the theorem by remarking that if $A \in \mathscr{M}(\mathscr{A})$, and if $A=U T=R+V S$, where $U T$ and $V S$ are polar decompositions, $R$ is bounded with $\|R\|=r$, and $\operatorname{tr} S<\infty$, then $\operatorname{tr}\left(T E_{r+2}^{1}\right) \leqq(r+1) \operatorname{tr}(S)$ (where $\left\{E_{\lambda}\right\}$ is the spectral family for $T$ ). For if $E_{r+2}^{\perp}=0$, this is immediate. Otherwise, if $v$ is any unit vector in the range of $E_{r+2}^{\perp},(r+2) \leqq\|R v\|+\|V S v\| \leqq r+\|S v\|$, so that $2 \leqq\|S v\|$, $E_{r+2}^{1} \cap F_{1}=0$ in consequence (writing $\left\{F_{\lambda}\right\}$ for the spectral family of $S$ ), and thus $E_{r+2}^{\perp} \prec F_{1}^{\perp}$, giving $\operatorname{tr}\left(E_{r+2}^{\perp}|R|^{2} E_{r+2}^{\perp}\right)^{1 / 2} \leqq r(\operatorname{tr} S)$, and, hence, $\operatorname{tr}\left(T E_{r+2}^{\perp}\right) \leqq(r+1) \operatorname{tr}(S)$.

THEOREM. Let $A \in \mathscr{M}(\mathscr{A})$ be the sum of a bounded operator and an operator whose absolute value has finite trace for an essential trace $\operatorname{tr}$ on $\mathscr{A}$. 
Then $\forall R>0 \quad \exists C_{R} \in \mathscr{A}^{+} \cap L(X)$ with measurable inverse such that the range of $C_{R}$ lies in the domain of $A^{k} \quad \forall k \geqq 0$, and $\left\|\left(z^{k} / k !\right) A^{k} C_{R}\right\|<2^{-k}$ for $|z|<R$, and $\sum_{k=0}^{\infty}(1 / k !) z^{k} A^{k} C_{R}$ is analytic on $|z|<R$ with values in $\mathscr{A}$. It is equal to $e^{z A} C_{R}$, where $e^{z A}$ is a function on the complex numbers to $\mathscr{M}(\mathscr{A})$ with $A e^{z A}=e^{z A} A, e^{\left(z_{1}+z_{2}\right) A}$ $=e^{z_{1} A} e^{z_{2} A}$, and $\left(e^{z A}\right)^{*}=e^{z_{A}^{*}}$.

The function $F(z)=e^{z A}$ from the complex plane to $\mathscr{M}(\mathscr{A})$ is uniquely characterized by the property that $F(0)=I$, and that $\forall R>0, \exists C_{R} \in \mathscr{A}^{+} \cap L(X)$ with measurable inverse such that the range of $C_{R}$ lies in the domains of $F(z)$ and $A F(z) \quad \forall z$ with $|z|<R$, and $F(z) C_{R}$ is analytic in $|z|<R$ with derivative $A F(z) C_{R}$.

Proof. Let $A=U T$ be the polar decomposition of $A$, and let $\left\{E_{\lambda}\right\}$ be the spectral family of $T$. Since $A$ is measurable, $\exists \lambda_{0}>0$ with $E_{\lambda_{0}}^{\perp}$ finite. By the remark we may assume that $\operatorname{tr}\left(T E_{\lambda_{0}}^{\perp}\right)<\infty$.

Choose $R>0$, and set $r=\left(4 R^{2}\right)^{-1}$. Let $Q_{n}^{k}$ be the null space of $E_{\lambda_{0}+(n+k) r}^{\perp} A^{n}$, so that $Q_{n}^{k \perp} \prec E_{\lambda_{0}+(n+k) r}^{\perp}$ and the range of $A^{n} Q_{n}^{k}$ lies in the range of $E_{\lambda_{0}+(n+k) r}$. Set $P_{k}=\bigcap_{n=0}^{\infty} Q_{n}^{k}$. Since $Q_{n}^{k+1} \geqq Q_{n}^{k}, P_{k} \uparrow, k=1,2, \ldots$

We have

$$
\begin{aligned}
\operatorname{tr} P_{k}^{\perp} & =\operatorname{tr}\left(\bigcup_{n=0}^{\infty} Q_{n}^{k \perp}\right) \leqq \sum_{n=0}^{\infty} \operatorname{tr} Q_{n}^{k \perp} \leqq \sum_{n=0}^{\infty} \operatorname{tr}\left(E_{\lambda_{0}+(n+k) r}^{\perp}\right) \\
& =\sum_{n=0}^{\infty}(1+n) \operatorname{tr}\left(E_{\lambda_{0}+(n+1+k) r}-E_{\lambda_{0}+(n+k) r}\right) \\
& \leqq r^{-1} \sum_{n=0}^{\infty}\left(\lambda_{0}+(n+k) r\right) \operatorname{tr}\left(E_{\lambda_{0}+(n+1+k) r}-E_{\lambda_{0}+(n+k) r}\right) \\
& \leqq r^{-1} \operatorname{tr}\left(T E_{\lambda_{0}+k r}^{\perp}\right) \downarrow 0 \text { as } k \rightarrow \infty,
\end{aligned}
$$

so that $P_{k} \uparrow I$ and $P_{k}$ is cofinite.

For fixed $k$,

$$
A^{n} P_{k}=\left(A E_{\lambda_{0}+(n-1+k) r}\right)\left(A E_{\lambda_{0}+(n-2+k) r}\right) \cdots\left(A E_{\lambda_{0}+k r}\right) P_{k}
$$

so that $\left\|A^{n} P_{k}\right\| \leqq \prod_{j=1}^{n}\left(\lambda_{0}+(j+(k-1)) r\right)$, so that

$$
\begin{aligned}
(1 / n !)\left\|A^{n} P_{k}\right\| & \leqq r^{n} \prod_{j=1}^{n}\left(\left(\lambda_{0} /(j+(k-1)) r\right)+1\right)(((k-1) / j)+1) \\
& \leqq r^{n} \prod_{j=1}^{n}((K / j)+1)^{2} \leqq r^{n} \exp \left(2 K \sum_{j=1}^{n}(1 / j)\right) \\
& \leqq r^{n} \exp (2 K(\ln n+1)) \leqq\left(e^{2 K}\right) r^{n / 2} \exp (2 K \ln n+(n / 2) \ln r) \\
& \leqq\left(e^{2 K}\right)(2 R)^{-n} \exp \left(2 K \ln n-(n / 2) \ln 4 R^{2}\right) \leqq K^{\prime}(R, k)(2 R)^{-n}
\end{aligned}
$$

(where $K$ depends on $\lambda_{0}, r$, and $k$; and $\lambda_{0}$ is considered forever fixed). Thus

$$
\left\|(1 / n !) z^{n} A^{n} P_{k}\right\| \leqq K^{\prime}(R, k) 2^{-n} \text { for }|z|<R .
$$

Set $C_{R}=\sum_{k=1}^{\infty} 2^{-k / 2}\left(K^{\prime}(R, k)+1\right)^{-1}\left(P_{k}-P_{k-1}\right)$, where $P_{0}=0$. Then $C_{R} \in \mathscr{A}^{+}$ $\cap L(X)$, and $C_{R}^{-1}$ is measurable because $P_{k}$ is cofinite for $k \geqq 1$. 
Let $x \in X$ have norm one, and set $x_{k}=\left(P_{k}-P_{k-1}\right) x$. Then

and

$$
C_{R} x=\sum_{k=1}^{\infty} 2^{-k / 2}\left(K^{\prime}(R, k)+1\right)^{-1} x_{k}
$$

$$
\begin{aligned}
\|(1 / n !) z^{n} A^{n} \sum_{k=1}^{M} 2^{-k / 2} & \left(K^{\prime}(R, k)+1\right)^{-1} x_{k} \| \\
& \leqq \sum_{k=1}^{M} 2^{-k / 2}\left(K^{\prime}(R, k)+1\right)^{-1}\left\|(1 / n !) z^{n} A^{n} x_{k}\right\| \\
& <\sum_{k=1}^{M} 2^{-k / 2}\left\|x_{k}\right\| 2^{-n} \\
& \leqq 2^{-n}\left(\sum_{k=1}^{\infty} 2^{-k}\right)^{1 / 2}\left(\sum_{k=1}^{\infty}\left\|x_{k}\right\|^{2}\right)^{1 / 2}=2^{-n}
\end{aligned}
$$

so that $\left\|(1 / n !) z^{n} A^{n} C_{R}\right\|<2^{-n} \quad \forall n \geqq 0, \forall z$ with $|z|<R$.

Thus $F\left(z, A, C_{R}\right)=\sum_{n=0}^{\infty}(1 / n !) z^{n} A^{n} C_{R}$ is an analytic function from $|z|<R$ into $\mathscr{A}$ with derivative $A F\left(z, A, C_{R}\right)$, and with $F\left(0, A, C_{R}\right)=C_{R}$.

Suppose $F(z)$ is a function on $|z|<R$ to $\mathscr{M}(\mathscr{A})$ with $F(0)=I$, and suppose that $\exists C \in \mathscr{A}^{+} \cap L(X)$ with measurable inverse such that the range of $C$ lies in the domains of $F(z)$ and $A F(z)$, and $F(z) C$ is analytic on $|z|<R$ with derivative $A F(z) C$.

Set $D=\left(\left(C^{-1}\right)^{2}+\left(C_{R}^{-1}\right)^{2}\right)^{-1}$. Then $C^{-1} D$ and $C_{R}^{-1} D$ are bounded. Consider $F\left(z, A, C_{R}\right) C_{R}^{-1} D-F(z) D$. This is analytic on $|z|<R$ with derivative

$$
A\left(F\left(z, A, C_{R}\right) C_{R}^{-1} D-F(z) D\right) \text {. }
$$

But it is equal to zero at $z=0$, so that all derivatives are also equal to zero at $z=0$, so that $F\left(z, A, C_{R}\right) C_{R}^{-1} D-F(z) D=0$ in $|z|<R$, and $F(z)=F\left(z, A, C_{R}\right) C_{R}^{-1}$ in $|z|<R$.

Thus $F\left(z, A, C_{R}\right) C_{R}^{-1}$ is independent of $R$. Denote it by $e^{z A}$. For any $x \in X$ in the range of $C_{R}$ which also has $A x$ in the range of $C_{R} \quad\left(A e^{z A}-e^{z A} A\right) x$ is an analytic function on $|z|<R$ which is equal to zero at $z=0$. Since its $n$th derivative is $A^{n}\left(A e^{z A}-e^{z A} A\right) x$, it is zero at $z=0$, so that $\left(A e^{z A}-e^{z A} A\right) x=0$ for all $x$ in the range of $C_{R}$ which have $A x$ also in the range of $C_{R}$, which means that $A e^{z A}-e^{2 A} A=0$ since such $x$ are dense in $X$.

Since $\left(e^{3 A} C_{R}\right)^{*}=C_{R}\left(e^{3 A}\right)^{*}$ has derivatives with respect to $z$ at zero equal to $C_{R} A^{* n}$, a uniqueness result exactly like the first one above, but with auxiliary operators applied on the left, gives $\left(e^{2 A}\right)^{*}=e^{2 A^{*}}$, or $\left(e^{2 A}\right)^{*}=e^{5 A^{*}}$.

Finally, consider $e^{z A} e^{z_{0} A}\left(\left|z_{0}\right|+|z|<R\right)$. For $x$ in the range of $C_{R}$ with $e^{z_{0} A} x$ in the range of $C_{R},\left(e^{z A} e^{z_{0} A}-e^{\left(z+z_{0}\right) A}\right) x$ is an analytic function in $|z|<R-\left|z_{0}\right|$ equal to zero at $z=0$ and with all derivatives thus equal to zero at $z=0$, so that $e^{z A} e^{z_{0} A}=e^{\left(z+z_{0}\right) A}$ by density of the $x$ 's.

Some weak generalization of the above theorem is possible. Let $A(\cdot)$ be a measurable function from a measure space $\Omega$ to unbounded operators affiliated with a ring $\mathscr{A}$, and let $P \in \mathscr{A}$ be a projection. Then the family of projections $Q \in \mathscr{A}$ with 
$P^{\perp} A(\cdot) Q=0$ a.e. forms an ascending net. Since the unit ball of $\mathscr{A}$ is metrizable in the strong topology ( $X$ is separable), there exists a sequence $\left\{Q_{n}\right\}$ of such projections converging strongly to the limit projection $Q_{0}$, so that $P{ }^{\perp} A(\cdot) Q_{0}=0$ a.e. Set $\theta_{A(\cdot)}(P)=Q_{0}$.

THEOREM. Let $A \in \mathscr{A}^{+} \cap L(X)$ have measurable inverse, and let $\mathrm{tr}$ be an essential trace on $\mathscr{A}$. Let $\mu$ be a measure on a right half line $[\alpha, \infty), \alpha>0$, for which $\int_{\alpha}^{\infty} \lambda d \mu(\lambda)<\infty$.

Then if $A(\cdot)$ is a measurable function from $[a, b]$ to $\mathscr{M}(\mathscr{A})$ such that

(1) $A(\cdot) A \in L^{1}(B(X),[a, b])$, and

(2) $\exists \varepsilon>0$ such that $\operatorname{tr}\left(\theta_{A \cdot(\cdot)}^{m}\left(E_{\lambda}^{\perp}\right)^{\perp}\right) \leqq \mu([1 / \lambda, \infty))$ for $\varepsilon>\lambda>0$ and $\forall m \geqq 0$, where $\left\{E_{\lambda}\right\}$ is the spectral family for $A$, then $\forall a \leqq s \leqq t \leqq b \quad \exists B(t, s) \in \mathscr{M}(\mathscr{A})$ with properties

(1) $B(t, t)=I \quad \forall t \in[a, b]$, and

(2) $\exists C \in \mathscr{A}^{+} \cap L(X)$ with measurable inverse such that $A^{-1} B(\cdot, s) C$ belongs to $L^{\infty}(B(X),[s, b])$, and such that $B(\cdot, s) C$ is absolutely continuous on $[s, b]$ and a.e. strongly differentiable with derivative $A(\cdot) B(\cdot, s) C$.

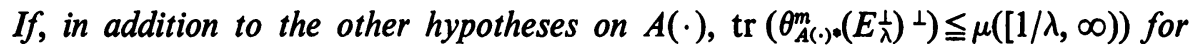
$\varepsilon>\lambda>0$ and $\forall m \geqq 0$, and $A A(\cdot)$ belongs to $L^{1}(B(X),[a, b])$, then $B(t, s)$ is uniquely characterized by the above two properties. $B(t, s)$ then has the additional properties

(3) $B\left(t, s_{0}\right) B\left(s_{0}, s\right)=B(t, s) \quad \forall a \leqq s \leqq s_{0} \leqq t \leqq b$,

(4) $\exists C^{\prime} \in \mathscr{A}^{+} \cap L(X)$ with measurable inverse such that $C^{\prime} B(t, \cdot) A(\cdot)$ belongs to $L^{1}(B(X),[a, t])$, and such that $C^{\prime} B(t, \cdot)$ is absolutely continuous on $[a, t]$ and a.e. strongly differentiable with derivative $-C^{\prime} B(t, \cdot) A(\cdot)$, and

(5) if $B_{1}(t, s)$ bears the same relation to $-A(\cdot)^{*}$ as $B(t, s)$ does to $A(\cdot)$, then $B_{1}(t, s)^{*}=B(t, s)^{-1}$.

Proof. We shall show that $A(\cdot)$ satisfies the hypotheses of Theorem 1 on the Cauchy problem for unbounded operators. We will set $C_{2}$ of that theorem equal to $I$.

Let $\lambda_{0}>1 / \varepsilon$ be such that $\int_{\lambda_{0}}^{\infty} \lambda d \mu(\lambda)<\infty$, where $\bar{E}_{\lambda}=E_{1 / \lambda}^{1}$ defines the spectral family of $A^{-1}$, set $Q_{n}^{k}=\theta_{A(\cdot)}^{n}\left(\bar{E}_{\lambda_{0}+(n+k) r}\right)$, and let $r=\left(2\|A(\cdot) A\|_{1}\right)^{-2}$. Let $P_{k}=\bigcap_{n=0}^{\infty} Q_{n}^{k}$. Since $Q_{n}^{k+1} \geqq Q_{n}^{k}$ (trivial for $n=0$, and then clear by induction), $P_{k} \uparrow, k=1,2, \ldots$

We have

$$
\begin{aligned}
\operatorname{tr} P_{k}^{\perp} & =\operatorname{tr}\left(\bigcup_{n=0}^{\infty} Q_{n}^{k \perp}\right) \leqq \sum_{n=0}^{\infty} \operatorname{tr} Q_{n}^{k \perp} \leqq \sum_{n=0}^{\infty} \mu\left(\left[\lambda_{0}+(n+k) r, \infty\right)\right) \\
& =\sum_{n=0}^{\infty}(1+n) \mu\left(\left[\lambda_{0}+(n+k) r, \lambda_{0}+(n+k+1) r\right)\right) \\
& \leqq r^{-1} \sum_{n=0}^{\infty}\left(\lambda_{0}+(n+k) r\right) \mu\left(\left[\lambda_{0}+(n+k) r, \lambda_{0}+(n+k+1) r\right)\right) \\
& \leqq r^{-1} \int_{\lambda_{0}+k r}^{\infty} \lambda d \mu(\lambda) \downarrow 0
\end{aligned}
$$

as $k \rightarrow \infty$, so that $P_{k} \uparrow I$, and $P_{k}$ is eventually cofinite. 
For almost all $t \in[a, b]$ the range of $A(t) Q_{n+1}^{k}$ lies in the range of $Q_{n}^{k+1}$, so that the range of $A(t) P_{k}$ lies in the range of $P_{k+1}$ for almost all $t \in[a, b]$. Thus for almost all $\left(t_{1}, \ldots, t_{m}\right) \in[a, b]^{m}$,

$$
\begin{aligned}
P_{i} A\left(t_{m}\right) P_{i} A\left(t_{m-1}\right) P_{i} & \cdots P_{i} A\left(t_{1}\right) P_{i} P_{k} \\
& =\left(P_{i} P_{k+m}\right) A\left(t_{m}\right)\left(P_{i} P_{k+m-1}\right) A\left(t_{m-1}\right) \cdots\left(P_{i} P_{k+1}\right) A\left(t_{1}\right) P_{i} P_{k},
\end{aligned}
$$

so that the range of $\left(P_{i} A\left(t_{m}\right) P_{i}\right)\left(P_{i} A\left(t_{m-1}\right) P_{i}\right) \cdots\left(P_{t} A\left(t_{1}\right) P_{i}\right) P_{k}$ lies in the range of $P_{k+m}$, and

$$
\begin{aligned}
\|\left(P_{i} A\left(t_{m}\right) P_{i}\right) \cdots & \left(P_{i} A\left(t_{1}\right) P_{i}\right) P_{k} \| \\
& \leqq\left\|A\left(t_{m}\right) P_{k+m-1}\right\| \cdots\left\|A\left(t_{1}\right) P_{k}\right\| \\
& \leqq\left\|A\left(t_{m}\right) A\right\|\left\|A^{-1} \bar{E}_{\lambda_{0}+(k+m-1) r}\right\| \cdots\left\|A\left(t_{1}\right) A\right\|\left\|A^{-1} \bar{E}_{\lambda_{0}+k r}\right\| \\
& \leqq\left(\prod_{j=1}^{m}\left(\lambda_{0}+(k-1+j) r\right)\right)\left\|A\left(t_{m}\right) A\right\| \cdots\left\|A\left(t_{1}\right) A\right\| \\
& \leqq m ! r^{m / 2} c(k)\left\|A\left(t_{m}\right) A\right\| \cdots\left\|A\left(t_{1}\right) A\right\| \\
& =c(k) m !\left(2\|A(\cdot) A\|_{1}\right)^{-m}\left\|A\left(t_{m}\right) A\right\| \cdots\left\|A\left(t_{1}\right) A\right\|
\end{aligned}
$$

as in the previous theorem.

Set $A_{n}(\cdot)=P_{n} A(\cdot) P_{n}$. Then $\left\|A_{n}(t)\right\| \leqq\left\|A(t) \bar{E}_{\lambda_{0}+k r}\right\| \leqq\|A(t) A\|\left\|A^{-1} \bar{E}_{\lambda_{0}+k r}\right\| \leqq$ $\left(\lambda_{0}+k r\right)\|A(t) A\|$, so that $A_{n}(\cdot) \in L^{1}(B(X),[a, b])$. Set

$$
C=\sum_{k=1}^{\infty} 2^{-k / 2}(c(k)+1)^{-1}\left(\lambda_{0}+k r+1\right)^{-1}\left(P_{k}-P_{k-1}\right),
$$

and let $C=C_{1}$ of Theorem 1. Let us verify hypotheses (1) and (2) of Theorem 1 .

We have $B_{n}(t, s)=I+\sum_{m=1}^{\infty} B_{n}^{m}(t, s)$, where $B_{n}^{m}(t, s)$ is defined by

$$
B_{n}^{m}(t, s) x=\int_{t \geq t_{m} \geq \ldots \geq t_{1} \geq s} A_{n}\left(t_{m}\right) \cdots A_{n}\left(t_{1}\right) x d t_{1} \cdots d t_{m} \quad \forall x \in X .
$$

Thus $B_{n}^{m}(t, s) P_{k}$ has range contained in the range of $P_{k+m}$, and

$$
\begin{aligned}
\left\|B_{n}^{m}(t, s) P_{k}\right\| & \leqq \int_{t \geq t_{m} \geq \ldots t_{1} \geq_{s}}\left\|A_{n}\left(t_{m}\right) \cdots A_{n}\left(t_{1}\right) P_{k}\right\| d t_{1} \cdots d t_{m} \\
& \leqq c(k) m !\left(2\|A(\cdot) A\|_{1}\right)^{-m} \int_{s}^{t}\left\|A\left(t_{m}\right) A\right\| d t_{m} \cdots \int_{s}^{t_{2}}\left\|A\left(t_{1}\right) A\right\| d t_{1} \\
& \leqq c(k) 2^{-m},
\end{aligned}
$$

so that, letting $\|x\|=1$ and $x_{k}=\left(P_{k}-P_{k-1}\right) x$, we get

$$
\begin{aligned}
\left\|A^{-1} B_{n}^{m}(t, s) C x\right\| & =\left\|A^{-1} \sum_{k=1}^{\infty} 2^{-k / 2}(c(k)+1)^{-1}\left(\lambda_{0}+k r+1\right)^{-1} B_{n}^{m}(t, s) P_{k} x_{k}\right\| \\
& \leqq \sum_{k=1}^{\infty} 2^{-k / 2}(c(k)+1)^{-1}\left(\lambda_{0}+k r+1\right)^{-1}\left\|A^{-1} \bar{E}_{\lambda_{0}+k r}\right\|\left\|B_{n}^{m}(t, s) P_{k}\right\|\left\|x_{k}\right\| \\
& \leqq 2^{-m} \sum_{k=1}^{\infty} 2^{-k / 2}\left\|x_{k}\right\| \leqq 2^{-m}
\end{aligned}
$$


so that $\left\|A^{-1} B_{n}^{m}(t, s) C\right\| \leqq 2^{-m} \quad \forall n$. It follows easily that $\left\|A^{-1} B_{n}(t, s) C\right\| \leqq 2 \quad \forall n$, $\forall a \leqq s \leqq t \leqq b$.

For hypothesis (3) of Theorem 1 we have $\left\|A_{n}(t) A\right\| \leqq\left\|A(t) P_{n} A\right\| \leqq\|A(t) A\|$. For hypothesis (4) we must show that $\left(A_{n}(t) A\right)^{*} \rightarrow(A(t) A)^{*}$ strongly for each $t \in[a, b]$. Since $\left\|\left(A_{n}(t) A\right)^{*}\right\|=\left\|A_{n}(t) A\right\| \leqq\|A(t) A\|$, it suffices to show strong convergence on a dense subspace of $X$. If $x$ is in the range of $P_{m}$, then for $n \geqq m,\left(A_{n}(t) A\right)^{*} x=$ $P_{n}(A(t) A)^{*} x$, which converges to $(A(t) A)^{*} x$.

We have only to set $B_{C_{1} C_{2}}(t, s) C^{-1}=B(t, s)$, where $B_{C_{1} C_{2}}(t, s)$ is as in Theorem 1 , and properties (1) and (2) of the present theorem are immediate.

Suppose now that $\operatorname{tr}\left(\theta_{A(\cdot) \cdot}^{m}\left(E_{\lambda}^{\perp}\right)^{\perp}\right) \leqq \mu([1 / \lambda, \infty))$ for $\varepsilon>\lambda>0$ and $\forall m \geqq 0$, and $A A(\cdot) \in L^{1}(B(X),[a, b])$. Let $\bar{A}(t)=A(t)$ for $a \leqq t \leqq b,=A(2 b-t)^{*}$ for $b<t \leqq 2 b-a$. Then $\bar{A}(\cdot) A \in L^{1}(B(X),[a, 2 b-a])$, and $\operatorname{tr}\left(\theta_{\bar{A}(\cdot)}^{m}\left(E_{\lambda}^{\perp}\right)^{\perp}\right) \leqq 2 \mu([1 / \lambda, \infty))$ for $\varepsilon>\lambda>0$ and $\forall m \geqq 0$. By following through the proof of the first part of this theorem we get that the $\bar{B}_{n}(t, s)=\bar{B}_{n}(2 b-s, 2 b-t)^{*}$ for $a \leqq s \leqq t \leqq b$ since $\bar{B}_{n}(t, s)=I+\sum_{m=1}^{\infty} \bar{B}_{n}^{m}(t, s)$, and $\bar{B}_{n}^{m}(t, s)$ is defined by

$$
\bar{B}_{n}^{m}(t, s) x=\int_{t \geqq t_{m} \geqq \ldots \geqq t_{1} \geqq s} \bar{A}_{n}\left(t_{m}\right) \cdots \bar{A}_{n}\left(t_{1}\right) x d t_{1} \cdots d t_{m},
$$

so that

$$
\begin{aligned}
\bar{B}_{n}^{m}(2 b-s, 2 b-t)^{*} x & =\int_{2 b-s \geqq t_{m} \geqq \ldots \geqq t_{1} \geqq 2 b-t} \bar{A}_{n}\left(t_{1}\right)^{*} \cdots \bar{A}_{n}\left(t_{m}\right)^{*} x d t_{1} \cdots d t_{m} \\
& =\int_{t \geqq t_{m} \geqq \ldots \geqq t_{1} \geqq s} \bar{A}_{n}\left(t_{m}\right) \cdots \bar{A}_{n}\left(t_{1}\right) x d t_{1} \cdots d t_{m} \quad \forall x \in X .
\end{aligned}
$$

Thus $\left\|A^{-1} \bar{B}_{n}(t, s) \bar{C}\right\| \leqq 2 \quad \forall a \leqq s \leqq t \leqq 2 b-a$, entails $\left\|\bar{C} \bar{B}_{n}(t, s)\right\| \leqq 2\|A\| \quad \forall a \leqq s$ $\leqq t \leqq b$.

To apply Theorem 2 on the Cauchy problem for unbounded operators and its corollaries to $\bar{B}(t, s), a \leqq s \leqq t \leqq b$, to demonstrate uniqueness and property (3) of this theorem, we need only set $C_{3}$ of Theorem 2 equal to $\bar{C}$ and show that $\bar{A}_{n}(t) A$ $\rightarrow \bar{A}(t) A$ strongly for fixed $t, a \leqq t \leqq b$. By uniform boundedness it suffices to show strong convergence on the range of each $\bar{P}_{k}$. But for $x$ with $\bar{P}_{k} x=x, \quad \forall n \geqq k$, $\bar{A}_{n}(t) A x=\bar{P}_{n} \bar{A}(t) \bar{P}_{n} A x=\bar{P}_{n} \bar{A}(t) A x \rightarrow \bar{A}(t) A x$.

Theorem 3 applies immediately to yield property (4) for $B(t, s)=\bar{B}(t, s)$, $a \leqq s \leqq t \leqq b$. Only property (5) remains to be verified. If we let $C_{1}$ and $C_{1}^{\prime}$ bear the same relation to $B_{1}(t, s)$ and $-A(\cdot)^{*}$ as $C$ and $C^{\prime}$ do to $B(t, s)$, we have that $C_{1} B_{1}(\cdot, s)^{*} B(\cdot, s) C x$ is absolutely continuous on $[s, b]$ and strongly differentiable with derivative $=0 \quad \forall x \in X$, so that $C_{1} B_{1}(t, s)^{*} B(t, s) C x=C_{1} C x \quad \forall t \in[s, b]$, and thus $B_{1}(t, s)^{*} B(t, s)=I \quad \forall a \leqq s \leqq t \leqq b$. Similarly, $C^{\prime} B(t, \cdot) B_{1}(t, \cdot)^{*} C_{1}^{\prime} x$ is absolutely continuous on $[a, t]$ and strongly differentiable with derivative $=0$ $\forall x \in X$, so that $C^{\prime} B(t, s) B_{1}(t, s)^{*} C_{1}^{\prime} x=C^{\prime} C_{1}^{\prime} x \quad \forall s \in[a, t]$, giving $B(t, s) B_{1}(t, s)^{*}$ $=I \quad \forall a \leqq s \leqq t \leqq b$, and thus $B_{1}(t, s)^{*}=B(t, s)^{-1}$.

Embellishment of the above theorem by allowing variable domains in the style 
of Theorems $1^{\prime}, 2^{\prime}$, and $3^{\prime}$ on the Cauchy problem for unbounded operators is very straightforward.

5. The Cauchy problem in simply-connected regions of the complex plane for operators in a Banach space. (a) We shall present what appear to be some basic theorems for bounded operators, but we make no attempt at a general treatment for unbounded operators. Indeed, we shall treat only one particular case for unbounded operators, having chosen it on grounds of simplicity and interest.

THEOREM. Let $R$ be a simply-connected region of the complex plane, and let $A(\cdot)$ be an analytic function from $R$ to $B(X)$. Then for all $z_{1}$ and $z_{2}$ in $R, \exists B\left(z_{1}, z_{2}\right) \in B(X)$ analytic in $z_{1}$ and such that $B(z, z)=I$ and $\left(\partial / \partial z_{1}\right) B\left(z_{1}, z_{2}\right)=A\left(z_{1}\right) B\left(z_{1}, z_{2}\right) . B\left(z_{1}, z_{2}\right)$ is uniquely characterized by these properties. It is also simultaneously analytic in $z_{1}$ and $z_{2}$, and has $\left(\partial / \partial z_{2}\right) B\left(z_{1}, z_{2}\right)=-B\left(z_{1}, z_{2}\right) A\left(z_{2}\right)$. It is invertible $\forall z_{1}, z_{2} \in R$, and $B\left(z_{1}, z_{2}\right) B\left(z_{2}, z_{3}\right)=B\left(z_{1}, z_{3}\right) \forall z_{1}, z_{2}$, and $z_{3} \in R$. Further, if the disc $\left|z-z_{0}\right| \leqq r$ is in $R$, then $\left\|B\left(z_{1}, z_{2}\right)\right\| \leqq e^{M r}$ for $\left|z_{1}-z_{0}\right|<r$ and $\left|z_{2}-z_{0}\right|<r$, where $M=$ $2 \sup _{\mid z-z_{0} \mathrm{i}<r}\|A(z)\|$.

Proof. Consider first a disc $\left|z-z_{0}\right| \leqq r$ contained in $R$. We have

$$
A(z)=\sum_{k=0}^{\infty} A_{k}\left(z-z_{0}\right)^{k}
$$

in $\left|z-z_{0}\right|<r, A_{k} \in B(X)$. Certainly

$$
B_{1}\left(z, z_{0}\right)=\sum_{k=0}^{\infty}(k+1)^{-1} A_{k}\left(z-z_{0}\right)^{k+1}
$$

is simultaneously analytic in $z$ and $z_{0}$ in $\left|z-z_{0}\right|<r, B_{1}\left(z_{0}, z_{0}\right)=0$, and $(\partial / \partial z) B_{1}\left(z, z_{0}\right)$ $=A(z)$. Similarly, we may start with $A(z) B_{1}\left(z, z_{0}\right)$ and find $B_{2}\left(z, z_{0}\right)$ simultaneously analytic in $z$ and $z_{0}$ in $\left|z-z_{0}\right|<r$ with $B_{2}\left(z_{0}, z_{0}\right)=0$ and with $(\partial / \partial z) B_{2}\left(z, z_{0}\right)=$ $A(z) B_{1}\left(z, z_{0}\right)$, and, in general, we may find $B_{n+1}\left(z, z_{0}\right)$ simultaneously analytic in $z$ and $z_{0}$ in $\left|z-z_{0}\right|<r$ such that $(\partial / \partial z) B_{n+1}\left(z, z_{0}\right)=A(z) B_{n}\left(z, z_{0}\right)$, and such that $B_{n+1}\left(z_{0}, z_{0}\right)=0$.

Then

Consider $\left\|B_{n}\left(z_{1}, z_{2}\right)\right\|$ for $\left|z_{1}-z_{0}\right|<r$ and $\left|z_{2}-z_{0}\right|<r$. Let $M=2 \sup _{\left|z-z_{0}\right|<r}\|A(z)\|$.

$$
B_{n+1}\left(z_{1} t+z_{2}(1-t), z_{2}\right)=\left(z_{1}-z_{2}\right) \int_{0}^{t} A\left(z_{1} s+z_{2}(1-s)\right) B_{n}\left(z_{1} s+z_{2}(1-s), z_{2}\right) d s,
$$

so that

$$
\left\|B_{n+1}\left(z_{1} t+z_{2}(1-t), z_{2}\right)\right\| \leqq(M r) \int_{0}^{t}\left\|B_{n}\left(z_{1} s+z_{2}(1-s), z_{2}\right)\right\| d s,
$$

and thus

$$
\left\|B_{1}\left(z_{1} t+z_{2}(1-t), z_{2}\right)\right\| \leqq(M r) t
$$

and

$$
\left\|B_{n}\left(z_{1} t+z_{2}(1-t), z_{2}\right)\right\| \leqq(M r t)^{n}(1 / n !) \leqq(M r)^{n}(1 / n !) .
$$


Thus $\left\|B_{n}\left(z_{1}, z_{2}\right)\right\| \leqq(1 / n !)(M r)^{n}$, and $B\left(z_{1}, z_{2}\right)=I+\sum_{k=1}^{\infty} B_{k}\left(z_{1}, z_{2}\right)$ is analytic in $z_{1}$ and $z_{2}$ for $\left|z_{1}-z_{0}\right|<r$ and $\left|z_{2}-z_{0}\right|<r$, with $\left\|B\left(z_{1}, z_{2}\right)-I\right\| \leqq e^{M r}-1, B\left(z_{1}, z_{1}\right)=I$, and $\left(\partial / \partial z_{1}\right) B\left(z_{1}, z_{2}\right)=A\left(z_{1}\right) B\left(z_{1}, z_{2}\right)$ for such $z_{1}$ and $z_{2}$.

Now let $z_{1}$ and $z_{2}$ be arbitrary in $R$, and let $z(t)$ be a piecewise-smooth path in $R$ with $z(0)=z_{2}$ and $z(1)=z_{1}$. Set

$$
B\left(z_{1}, z_{2}\right)=B\left(z\left(t_{n}\right), z\left(t_{n-1}\right)\right) \cdots B\left(z\left(t_{1}\right), z\left(t_{0}\right)\right),
$$

where $0=t_{0} \leqq t_{1} \leqq \cdots \leqq t_{n}=1$, and the $B\left(z\left(t_{i}\right), z\left(t_{i-1}\right)\right)$ are as determined above. By the general results on bounded operators applied to $z^{\prime}(\cdot) A(z(\cdot)), B\left(z_{1}, z_{2}\right)$ is independent of the subdivision taken. Further, by the usual deformation arguments, it is independent of the path $z(t)$ chosen. Uniqueness follows from the general results on bounded operators, and analyticity in $z_{1}$ and $z_{2}$ are direct from the expression of $B\left(z_{1}, z_{2}\right)$. The other statements follow from $B\left(z_{1}, z_{2}\right) B\left(z_{2}, z_{3}\right)$ $=B\left(z_{1}, z_{3}\right)$.

Behavior toward the boundary of $R$ is of some interest. Perhaps the simplest theorem of this sort is the one that follows. Note first that if $R$ is a convex region with closure $\bar{R}$, then any piecewise-smooth path $\gamma(t), 0 \leqq t \leqq 1$, in $\bar{R}$ may be approximated by polygonal paths $\left\{\gamma_{n}(t)\right\}$ in $R$ in such fashion that $\left\|\gamma(\cdot)-\gamma_{n}(\cdot)\right\|_{\infty} \rightarrow 0$, and $\left\|\gamma^{\prime}(\cdot)-\gamma_{n}^{\prime}(\cdot)\right\|_{1} \rightarrow 0$.

THEOREM. Let $R$ be a convex region of the complex plane, and let $A(\cdot)$ be analytic from $R$ to $B(X)$. Let $B\left(z_{1}, z_{2}\right)$ be as in the above theorem.

Suppose $\bar{A}(\cdot)$ is a function on the closure $\bar{R}$ of $R$ to $B(X)$ with $\bar{A}(z)=A(z) \quad \forall z \in R$ and with $\bar{A}(\cdot)$ continuous from $\bar{R}$ to the Banach space $B(X)$.

Then $\exists \bar{B}\left(z_{1}, z_{2}\right)$ continuous from $\bar{R} \times \bar{R}$ to $B(X)$ with $\bar{B}\left(z_{1}, z_{2}\right)=B\left(z_{1}, z_{2}\right) \quad \forall z_{1}$, $z_{2} \in R . \bar{B}(z, z)=I \quad \forall z \in \bar{R}$, and $\bar{B}\left(z_{1}, z_{2}\right) \bar{B}\left(z_{2}, z_{3}\right)=\bar{B}\left(z_{1}, z_{3}\right) \quad \forall z_{1}, z_{2}$, and $z_{3} \in \bar{R}$. If $\gamma(t), 0 \leqq t \leqq 1$, is any piecewise-smooth path in $\bar{R}$, then $\bar{B}(\gamma(\cdot), \gamma(s))$ is absolutely continuous on $[s, 1]$, and uniformly differentiable with derivative $\gamma^{\prime}(\cdot) \bar{A}(\cdot) \bar{B}(\gamma(\cdot), \gamma(s)$ ) a.e. on [s, 1]. Similarly, $\bar{B}(\gamma(t), \gamma(\cdot))$ is absolutely continuous on $[0, t]$, and uniformly differentiable with derivative $-\bar{B}(\gamma(t), \gamma(\cdot)) \bar{A}(\cdot) \gamma^{\prime}(\cdot)$ a.e.

Proof. Let $z_{1}$ and $z_{2}$ be points of $\bar{R}$, and let $\gamma(t), 0 \leqq t \leqq 1$, be a simple piecewisesmooth path in $R$ for $0<t<1$ with $\gamma(0)=z_{2}$ and $\gamma(1)=z_{1}$. Define $\bar{B}(\gamma(t), \gamma(s))$ for $0 \leqq s \leqq t \leqq 1$ by $\bar{B}(\gamma(t), \gamma(t))=I$, and $\bar{B}(\gamma(\cdot), \gamma(s))$ is absolutely continuous on [s, 1] and a.e. uniformly differentiable with derivative $\gamma^{\prime}(\cdot) \bar{A}(\cdot) \bar{B}(\gamma(\cdot), \gamma(s))$. Then $\bar{B}(\gamma(t), \gamma(s))$ is jointly uniformly continuous in $t$ and $s$, and $\bar{B}(\gamma(t), \gamma(s))$ $=B(\gamma(t), \gamma(s))$ for $0<s \leqq t<1$, so that $B(\gamma(t), \gamma(s))$ converges uniformly to $\bar{B}(\gamma(1), \gamma(0))$ as $t \uparrow 1$ and $s \downarrow 0$. Set $\bar{B}\left(z_{1}, z_{2}\right)=\bar{B}(\gamma(1), \gamma(0))$.

It is easy to see that this definition is independent of the path chosen, and that $\bar{B}\left(z_{1}, z_{2}\right)$ is continuous on $\bar{R} \times \bar{R}$. The other properties are straightforward.

(b) In at least one special case we may prove analogous theorems for unbounded operators. We let $\mathscr{A}$ be a weakly-closed ring of operators over a Hilbert space $X$ with center $\mathscr{Z}$. An operator in $C D(X)$ affiliated with $\mathscr{A}$ is measurable with respect 
to $\mathscr{Z}$ if there exists a sequence of central projections $P_{n} \uparrow I$ in the domain of the operator. We call the ${ }^{*}$-algebra of such operators $\mathscr{M}(\mathscr{A}, \mathscr{Z})$ (clearly such an operator is the closure of its restriction to the ranges of any sequence of central projections in its domain converging monotonely upwards to the identity).

THEOREM. Let $R$ be a simply-connected region of the complex plane, and let $A(\cdot)$ be a function from $R$ into $\mathscr{M}(\mathscr{A}, \mathscr{Z})$.

Suppose that $\forall z_{0} \in R, \exists \varepsilon>0$ and $A \in \mathscr{Z}^{+} \cap L(X)$ such that $A(\cdot) A$ is analytic in $\left|z-z_{0}\right|<\varepsilon$. Then $\forall z_{1}, z_{2} \in R \quad \exists B\left(z_{1}, z_{2}\right) \in \mathscr{M}(\mathscr{A}, \mathscr{Z})$ with the following properties:

(1) if $R_{0}$ is a subregion of $R$ with compact closure $\bar{R}_{0} \subset R, \quad \exists C \in \mathscr{Z}^{+} \cap L(X)$ such that $B\left(z_{1}, z_{2}\right) C \in \mathscr{A} \quad \forall z_{1}, z_{2} \in R_{0}$,

(2) $B\left(z_{1}, z_{2}\right) C$ is analytic in $z_{1}$ for $z_{1}, z_{2} \in R_{0}$, and

$$
\left(\partial / \partial z_{1}\right)\left(B\left(z_{1}, z_{2}\right) C\right)=A\left(z_{1}\right) B\left(z_{1}, z_{2}\right) C,
$$

(3) $B(z, z)=I \quad \forall z \in R$.

These properties uniquely characterize $B\left(z_{1}, z_{2}\right)$. Moreover, $B\left(z_{1}, z_{2}\right) C$ is simultaneously analytic in $z_{1}$ and $z_{2}$ over $R_{0}$ with $\left(\partial / \partial z_{2}\right)\left(B\left(z_{1}, z_{2}\right) C\right)=-B\left(z_{1}, z_{2}\right) A\left(z_{2}\right) C$, and $B\left(z_{1}, z_{2}\right) B\left(z_{2}, z_{3}\right)=B\left(z_{1}, z_{3}\right) \quad \forall z_{1}, z_{2}$, and $z_{3} \in R$.

Proof. Let $R_{0}$ be a simply-connected subregion of $R$ with compact closure $\bar{R}_{0} \subset R$, and let $A_{1}, \ldots, A_{n} \in \mathscr{Z}^{+} \cap L(X)$ be such that $A(\cdot) A_{i}$ is analytic in $\left|z-z_{i}\right|$ $<\varepsilon_{i}$, where the discs $\left|z-z_{i}\right|<\varepsilon_{i} / 2$ cover $\bar{R}_{0}$. Set $A=A_{1} \cdots A_{N} \in \mathscr{Z}^{+} \cap L(X)$. Then $A(\cdot) A$ is analytic in a neighborhood of $\bar{R}_{0}$.

If $\left\{E_{\lambda}\right\}$ is the spectral family for $A$, set $P_{n}=E_{1 / n}^{1}$. Letting

$$
a_{n}=\sup \left\{\left\|A(z) P_{n}\right\|:\left|z-z_{i}\right| \leqq \varepsilon_{i} / 2 \text { for some } i\right\} \text { and } M=\sum_{i=1}^{N} \varepsilon_{i},
$$

set

$$
C_{1}=\frac{1}{2} e^{-M a_{1}} P_{1}+\sum_{n=2}^{\infty} 2^{-n} e^{-M a_{n}}\left(P_{n}-P_{n-1}\right), \text { and } C=C_{1} A .
$$

Since $A_{n}(\cdot)=A(\cdot) P_{n}$ is analytic in $R_{0}, \exists B_{n}\left(z_{1}, z_{2}\right) \in \mathscr{A}$ as in the preceding theorem jointly analytic in $z_{1}$ and $z_{2}$, and with $\left(\partial / \partial z_{1}\right) B_{n}\left(z_{1}, z_{2}\right)=A\left(z_{1}\right) P_{n} B_{n}\left(z_{1}, z_{2}\right)$, $\left(\partial / \partial z_{2}\right) B_{n}\left(z_{1}, z_{2}\right)=-B_{n}\left(z_{1}, z_{2}\right) A\left(z_{2}\right) P_{n}$, and $B_{n}\left(z_{1}, z_{2}\right) B_{n}\left(z_{2}, z_{3}\right)=B_{n}\left(z_{1}, z_{3}\right)$. By following paths through the discs $\left|z-z_{i}\right|<\varepsilon_{i} / 2$, we have $\left\|B_{n}\left(z_{1}, z_{2}\right)\right\| \leqq \exp \left(a_{n} \sum_{i=1}^{N} \varepsilon_{i}\right)$ $=e^{M a_{n}}$. By uniqueness $B_{n}\left(z_{1}, z_{2}\right)=\left(I-P_{n}\right)+P_{n} B_{n}\left(z_{1}, z_{2}\right)$, and $P_{n} B_{m}\left(z_{1}, z_{2}\right)=$ $P_{n} B_{n}\left(z_{1}, z_{2}\right)$ for $m \geqq n$. Then for $m>n>n_{0}$,

$$
\begin{aligned}
\left\|A^{-1}\left(B_{m}\left(z_{1}, z_{2}\right)-B_{n}\left(z_{1}, z_{2}\right)\right) C\right\| & =\left\|\left(B_{m}\left(z_{1}, z_{2}\right)-B_{n}\left(z_{1}, z_{2}\right)\right) C_{1}\right\| \\
& \leqq\left\|\left(P_{m}-P_{n}\right) C_{1}\right\|+\left\|\left(P_{m} B_{m}\left(z_{1}, z_{2}\right)-P_{n} B_{n}\left(z_{1}, z_{2}\right)\right) C_{1}\right\| \\
& \leqq 2 \sum_{j=n_{0}}^{\infty} 2^{-1},
\end{aligned}
$$

so that $\left\{A^{-1} B_{n}\left(z_{1}, z_{2}\right) C\right\}$ is uniformly Cauchy over $R_{0} \times R_{0}$, and therefore converges to an analytic $f\left(z_{1}, z_{2}\right)$. 
Set $B\left(z_{1}, z_{2}\right)=C^{-1} A f\left(z_{1}, z_{2}\right)$. Then $B(z, z)=I$, and $B\left(z_{1}, z_{2}\right) C \in \mathscr{A}$ is simultaneously analytic in $z_{1}$ and $z_{2}$ over $R_{0}$. By evaluating derivatives on projections $P_{n}$, we have that $\left(\partial / \partial z_{1}\right) B\left(z_{1}, z_{2}\right) C=A\left(z_{1}\right) B\left(z_{1}, z_{2}\right) C$, and $\left(\partial / \partial z_{2}\right) B\left(z_{1}, z_{2}\right) C=$ $-B\left(z_{1}, z_{2}\right) A\left(z_{2}\right) C$. Also by considering behavior on projections $P_{n}$,

$$
\left(B\left(z_{1}, z_{2}\right) C\right)\left(B\left(z_{2}, z_{3}\right) C\right)=B\left(z_{1}, z_{3}\right) C^{2},
$$

so that $B\left(z_{1}, z_{2}\right) B\left(z_{2}, z_{3}\right)=B\left(z_{1}, z_{3}\right)$.

If $\bar{B}\left(z_{1}, z_{2}\right)$ defined on $R_{0}$ with values in $\mathscr{M}(\mathscr{A}, \mathscr{Z})$ has $\bar{B}(z, z)=I$, and $\bar{B}\left(z_{1}, z_{2}\right) \bar{C}$ analytic in $z_{1}$ with derivative $A\left(z_{1}\right) \bar{B}\left(z_{1}, z_{2}\right) \bar{C}$ for some $\bar{C} \in \mathscr{Z}^{+} \cap L(X)$, then $C B\left(z_{2}, z_{1}\right) \bar{B}\left(z_{1}, z_{2}\right) \bar{C}$ is analytic in $z_{1}$ with derivative equal to zero, so that $C B\left(z_{2}, z_{1}\right) \bar{B}\left(z_{1}, z_{2}\right) \bar{C}=C \bar{C} \quad \forall z_{1}, z_{2} \in R_{0}$, so that $B\left(z_{2}, z_{1}\right) \bar{B}\left(z_{1}, z_{2}\right)=I \quad \forall z_{1}, z_{2} \in R_{0}$, and, hence, $B\left(z_{1}, z_{2}\right) B\left(z_{2}, z_{1}\right) \bar{B}\left(z_{1}, z_{2}\right)=\bar{B}\left(z_{1}, z_{2}\right)=B\left(z_{1}, z_{2}\right) \forall z_{1}, z_{2} \in R_{0}$. Thus $B\left(z_{1}, z_{2}\right)$ is uniquely defined independently of the construction used.

Using the same notations, we may prove a theorem on values at the boundary of $R$.

THEOREM. Let $R$ be a convex region of the complex plane with closure $\bar{R}$, and let $A(\cdot)$ and $B\left(z_{1}, z_{2}\right)$ be as in the previous theorem.

Suppose there exists a function $\bar{A}(\cdot)$ on $\bar{R}$ with values in $\mathscr{M}(\mathscr{A}, \mathscr{Z})$ such that $\bar{A}(z)=A(z) \quad \forall z \in R$, and such that $\bar{A}(\cdot)$ is continuous on $\bar{R}$ in the following sense: $\forall z_{0} \in \bar{R}$, there exists a sequence $\left\{P_{n}\right\}$ of projections in $\mathscr{Z}$ with $P_{n} \uparrow I, \bar{A}\left(z_{0}\right) P_{n} \in \mathscr{A} \quad \forall n$, and with $\bar{A}\left(z_{j}\right) P_{n} \rightarrow \bar{A}\left(z_{0}\right) P_{n}$ uniformly for each $n$ and each sequence $\left\{z_{j}\right\}$ in $\bar{R}$ with $z_{j} \rightarrow z_{0}$.

Then there exists a function $\bar{B}\left(z_{1}, z_{2}\right)$ on $\bar{R} \times \bar{R}$ with values in $\mathscr{M}(\mathscr{A}, \mathscr{Z})$ equal to $B\left(z_{1}, z_{2}\right)$ on $R \times R$ and continuous on $\bar{R} \times \bar{R}$ in the sense just described for $\bar{A}(\cdot)$. $\bar{B}(z, z)=I \quad \forall z \in \bar{R}, \bar{B}\left(z_{1}, z_{2}\right) \bar{B}\left(z_{2}, z_{3}\right)=\bar{B}\left(z_{1}, z_{3}\right) \quad \forall z_{1}, z_{2}$, and $z_{3} \in \bar{R}$, and if $\gamma(t)$ is any piecewise-smooth path in $\bar{R}, 0 \leqq t \leqq 1$, there exists a sequence of projections $\left\{P_{n}\right\}$ in $\mathscr{Z}$ with $P_{n} \uparrow I$ for each $0 \leqq s_{0} \leqq t_{0} \leqq 1$ such that $\bar{B}\left(\gamma(\cdot), \gamma\left(s_{0}\right)\right) P_{n}$ and $\bar{B}\left(\gamma\left(t_{0}\right), \gamma(\cdot)\right) P_{n}$ are absolutely continuous in neighborhoods of $t_{0}$ and $s_{0}$, respectively, where the neighborhoods depend on $n$, and they are a.e. uniformly differentiable in these neighborhoods with

$$
(\partial / \partial t) \bar{B}\left(\gamma(t), \gamma\left(s_{0}\right)\right) P_{n}=\gamma^{\prime}(t) \bar{A}(t) \bar{B}\left(\gamma(t), \gamma\left(s_{0}\right)\right) P_{n},
$$

and

$$
(\partial / \partial s) \bar{B}\left(\gamma\left(t_{0}\right), \gamma(s)\right) P_{n}=-\bar{B}\left(\gamma\left(t_{0}\right), \gamma(s)\right) \bar{A}(\gamma(s)) \gamma^{\prime}(s) P_{n} .
$$

Proof. Let $z_{1}$ and $z_{2} \in \bar{R}$, and choose a sequence $\left\{P_{n}\right\}$ of projections in $\mathscr{Z}$ with $P_{n} \uparrow I$ satisfying the needs of the definition of continuity of $\bar{A}(\cdot)$ at $z_{1}$ and $z_{2}$. Let $\gamma(t), 0 \leqq t \leqq 1$, be a piecewise-smooth path in $\bar{R}$ with $\gamma(t) \in R$ for $0<t<1$, and $\gamma(0)=z_{2}, \gamma(1)=z_{1}$.

Fix $n$, and choose $\varepsilon$ such that $\bar{A}(\cdot) P_{n}$ is uniformly continuous on neighborhoods $\left|z-z_{1}\right|<\varepsilon$ and $\left|z-z_{2}\right|<\varepsilon$ in $\bar{R}$. Choose $\delta>0$ such that $\left|\gamma(t)-z_{1}\right|<\varepsilon$ for $1-\delta \leqq t \leqq 1$, and $\left|\gamma(t)-z_{2}\right|<\varepsilon$ for $0 \leqq t \leqq \delta$. Define $f_{1}(\cdot)$ by $f_{1}(1-\delta)=I, f_{1}(\cdot)$ is absolutely 
continuous on $[1-\delta, 1]$, and $f_{1}(\cdot)$ is a.e. uniformly differentiable on $[1-\delta, 1]$ with derivative $\gamma^{\prime}(\cdot) \bar{A}(\cdot) P_{n} f_{1}(\cdot)$. Define $f_{0}(\cdot)$ by $f_{0}^{\prime}(0)=I, f_{0}(\cdot)$ is absolutely continuous on $[0, \delta]$, and $f_{0}(\cdot)$ is a.e. uniformly differentiable on $[0, \delta]$ with derivative $\gamma^{\prime}(\cdot) \bar{A}(\cdot) P_{n} f_{0}(\cdot)$.

Set $B_{n}\left(z_{1}, z_{2}\right)=f_{1}(1) B(\gamma(1-\delta), \gamma(\delta)) f_{0}(\delta) P_{n}$. It is not hard to see that $B_{n}\left(z_{1}, z_{2}\right)$ depends only on the projection $P_{n}$, and that $P_{n} B_{m}\left(z_{1}, z_{2}\right)=B_{n}\left(z_{1}, z_{2}\right)$ for $m \geqq n$.

Define $\bar{B}\left(z_{1}, z_{2}\right)$ by $\bar{B}\left(z_{1}, z_{2}\right)\left(P_{n+1}-P_{n}\right)=B_{n+1}\left(z_{1}, z_{2}\right)-B_{n}\left(z_{1}, z_{2}\right)$. Then $\bar{B}\left(z_{1}, z_{2}\right)$ $\in \mathscr{M}(\mathscr{A}, \mathscr{Z})$. By the usual uniqueness properties and taking refinements of sequences of projections, we see that the definition of $\bar{B}\left(z_{1}, z_{2}\right)$ is really independent of the choice of $\left\{P_{n}\right\}$. The properties claimed for $\bar{B}\left(z_{1}, z_{2}\right)$ follow by application of suitable projections.

For an example of the theorem just proved let us take $X=L^{2}([0,1]), \mathscr{A}=$ the ring of multiplications by bounded measurable functions, $R=$ the square $(0,1) \times(0,1)$, and $A(z)$ the operator defined by $(A(z) f)(x)=(x-z)^{-1} f(x)$ for $f \in L^{2}([0,1])$. Then $\left(B\left(z_{1}, z_{2}\right) f\right)(x)=\left(\left(x-z_{2}\right) /\left(x-z_{1}\right)\right) f(x)$. The extensions $\bar{A}(\cdot)$ and $\bar{B}\left(z_{1}, z_{2}\right)$ to $[0,1] \times[0,1]$ are defined by the same formulas.

\section{BiBLIOGRAPHY}

1. J. Dixmier, Les algèbres d'opérateurs dans l'espace Hilbertien, Gauthier-Villars, Paris, 1957.

2. E. Hille and R. Phillips, Functional analysis and semi-groups, rev. ed., Amer. Math. Soc. Colloq. Publ. Vol. 31, Amer. Math. Soc., Providence, R. I., 1957.

3. T. Kato, Integration of the equation of evolution in a Banach space, J. Math. Soc. Japan 5 (1954), 208-234.

4. - On linear differential equations in Banach spaces, Comm. Pure Appl. Math. 9 (1956), 479-486.

5. F. Riesz and B. von Sz.-Nagy, Functional analysis, Ungar, New York, 1955.

6. I. E. Segal, A non-commutative extension of abstract integration, Ann. of Math. 57 (1953), 401-457.

UNIVERSITY OF WASHINGTON, Seattre, Washington 